\title{
Particle Physics confronts the solar neutrino problem
}

\author{
Palash B. Pal \\ Institute of Theoretical Science \\ University of Oregon, Eugene, OR 97403, USA
}

$\mathrm{DOE} / \mathrm{ER} / 40224--205$

DE92 014013

\begin{abstract}
This review has four parts. In Part I, we describe the reactions that produce reutrinos in the sun and the expected flux of those neutrinos on the earth. We then discuss the detection of these neutrinos, and how the results obtained differ from the theoretical expectations, leading to what is known as the solar neutrino problem. In Part II, we show how neutrino oscillations can provide a solution to the solar neutrino problem. This includes vacuum oscillations, as well as matter enhanced oscillations. In Part III, we discr's the possibility of time variation of the neutrino flux and how a magnetic moment of the neutrino can solve the problem. We also discuss particle physics models which can give rise to the required values of magnetic moments. In Part IV, we present some concluding remarks and outlook for the recent future.
\end{abstract}

\section{DISCLAIMER}

This report was prepared as an account of work sponsored by an agency of the United States Government. Neither the United States Government nor any agency thereof, nor any of their employees, makes any warranty, express or implied, or assumes any legal liability or responsibility for the accuracy, completeness, or usefulness of any information, apparatus, product, or process disclosed, or represents that its use would not infringe privately owned rights. Reference herein to any specific commercial product, process, or service by trade name, trademark, manufacturer, or otherwise does not necessarily constitute or imply its endorsemert, recommendation, or favoring by the United States Government or any agency thereof. The views and opinions of authors expressed herein do not necessarily state or reflect those of the United States Government or any agency thereof. 


\section{Contents}

I The solar neutrino problem 5

1 Source of solar neutrinos 6

2 Detection of solar neutrinos 9

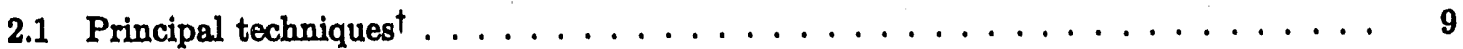

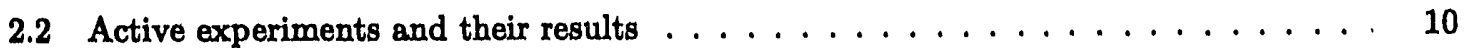

2.2 .1 The ${ }^{37} \mathrm{Cl}$ experiment . . . . . . . . . . . . . . . . . . . 10

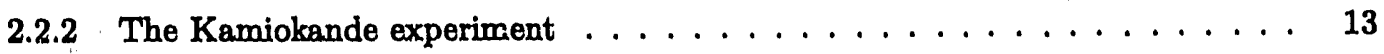

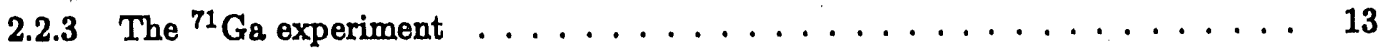

II Neutrino oscillation $\quad 13$

3 Vacuum neutrino oscillations and the solar neutrino problem 14

3.1 Hamiltonian formulation of vacuum neutrino oscillations . . . . . . . . . . . . 14

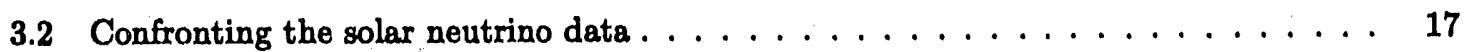

4 Neutrino oscillation in uniform matter 18

5 Resonant neutrino conversion 26

5.1 Motion of neutrinos in non-uniform matter $\ldots \ldots \ldots \ldots$

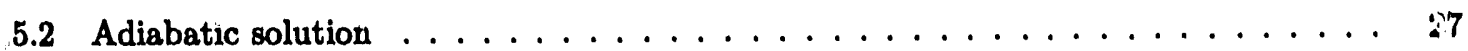

5.3 Non-adiabatic effects . . . . . . . . . . . . . . . . . . . . . 29

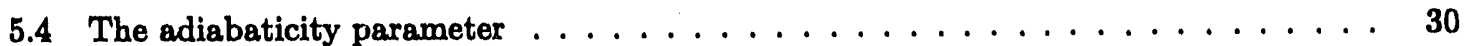

5.5 Evaluation of the jumping probability ${ }^{\dagger} \ldots \ldots \ldots \ldots \ldots \ldots \ldots$

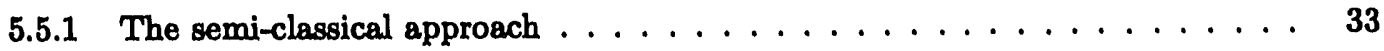

5.5.2 The extreme non-adiabatic limit $\ldots \ldots \ldots \ldots \ldots \ldots \ldots \ldots \ldots \ldots$

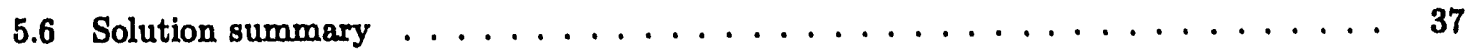

6 Resonant neutrino oscillation confronts the solar neutrino data 38

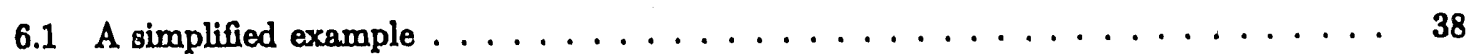

6.2 The full analysis $\ldots \ldots \ldots \ldots \ldots \ldots \ldots \ldots$

7 Variations on the theme ${ }^{\dagger}$. 45

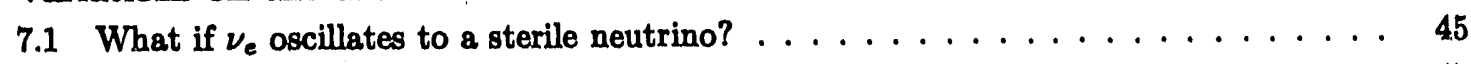

7.2 Solution with more than two generations of neutrinos $\ldots \ldots \ldots \ldots$

III Neutrino magnetic moment $\quad 50$

8 Kinematics of neutrino propagation in magnetic field 52

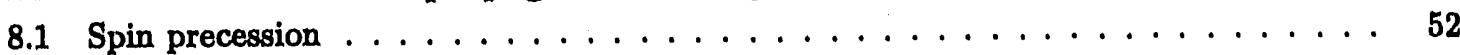

8.2 Spin-flavor oscillations and resonance ${ }^{\dagger} \ldots \ldots \ldots \ldots \ldots \ldots \ldots$

8.3 How large a magnetic moment do we need? . . . . . . . . . . . 56 
9 Easy models for neutrino magnetic moment 5 T

9.1 The simplest attempt $\ldots \ldots \ldots \ldots \ldots \ldots \ldots \ldots \ldots \ldots$

9.2 Naive Higgs models $\ldots \ldots \ldots \ldots \ldots \ldots \ldots \ldots$

10 Models with naturally large magnetic moments 60

10.1 Generic problem with large magnetic moment . . . . . . . . . . . . . . 60

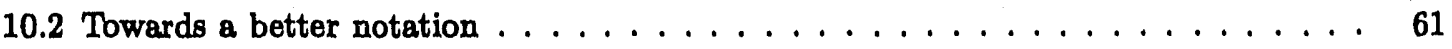

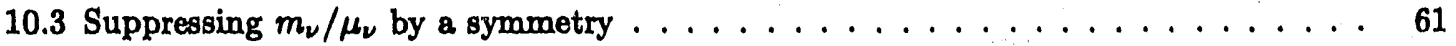

10.4 Implementing the Voloshin symmetry $\ldots \ldots \ldots \ldots \ldots \ldots$

10.4.1 Example: Extended electroweak symmetry ${ }^{\dagger} \ldots \ldots \ldots \ldots$. . . . . . . 63

10.4.2 Example: Quaternionic group ${ }^{\dagger} \ldots \ldots \ldots \ldots \ldots$

11 Other ways of getting a large magnetic moment 67

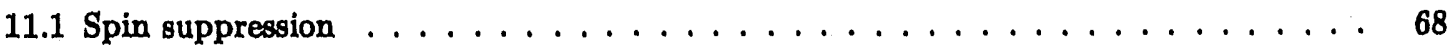

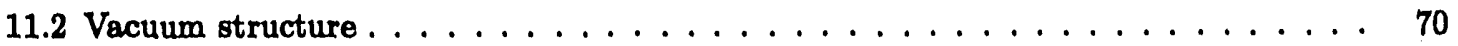

12 Chlorine vs Kamioka 71

IV Concluding remarks $\quad 71$

13 Outlook for future experiments

14 Outlook for Physics beyond the standard model 73

$\begin{array}{ll}\text { Appendices } & \mathbf{7 5}\end{array}$

$\begin{array}{ll}\text { A Laboratory bounds on neutrino oscillation parameters } & 75\end{array}$

B More rigorous derivation of dispersion relations in medium 75

$\begin{array}{ll}\text { C Laboratory bounds on neutrino magnetic moments } & \mathbf{7 7}\end{array}$

$\begin{array}{ll}\text { References } & \mathbf{7 8}\end{array}$

The sections marked with a ${ }^{\dagger}$ sign can be skipped in the first reading. 


\section{Part I}

\section{The solar neutrino problem}

The sun is a huge nuclear reactor where lots of reactions are going on, synthesizing hydrogen into helium and subsequently to heavier elements ${ }^{\sharp 1}$. In these reactions, some neutrinos are created, as we describe in more detail below. The physics of the sun is supposed to be quite well understood to estimate the rate of production of these neutrinos. We can try to detect these neutrinos on the earth. The experiments performed so far have detected much less neutrinos than were theoretically expected. This is called the Solar neutrino problem. There are many other aspects of the problem which will be elaborated as we go on.

Logically, the problem must be in one of the following aspects: (1) the detection of neutrinos might be faulty; (2) the calculation of expected neutrino fluxes might be wrong because of poorly known input parameters and uncertainties in the calculation with the standard solar model; (3) something might be lacking in our understanding of the neutrino properties.

Though all these alternatives seemed equally likely at the time the first solar neutrino experiments were performed, it now seems that the first alternative is ruled out because since then, other experiments have been performed, employing different detection tachniques, and they also find less neutrinos than expected. As for the second alternative, all we have to say is that Bahcall and his collaborators have checked and improved their calculations over the years and their estimates of their errors have progressively become smaller. Recently, another group has performed the same calculation [2] and found results close to that of Bahcall and collaborators [3], although their central value is somewhat lower, i.e., closer to the flux seen in the experiments. There has also been various discussions about modifying the standard solar model which predict lower values for neutrino flux. We will not discuss these possibilities here. It has been argued [4] that if the neutrino properties are described exactly by the standard electroweak model, changes in the solar model only cannot explain the discrepancy between the rate of neutrinos detected in different experiments. In this review, we take the other extreme viewpoint, viz., assume that the standard solar model is correct, and the calculations of Bahcall's group [3] are accurate. Thus, there is indeed a discrepancy between solar model calculations and experimental observations. The purpose of this review is to discuss the theoretical ideas put forward to understand this problem by some properties of

\footnotetext{
For a textbook presentation of the solar astrophysics, see Ref. [1].
} 
neutrinos which are so far untested by laboratory experiments. An outline of the organization is provided in the abstract.

\section{Source of solar neutrinos}

The dominant chain of reactions taking place in the sun can be summarized by the equation:

$$
4 p \rightarrow{ }^{4} \mathrm{He}+2 e^{+}+2 \nu_{e}+25 \mathrm{MeV} .
$$

This, of course, is not just one simple reaction, but rather takes place in many steps, as elaborated later. The energy released manifests mainly as photons, which undergo many scatterings before they come out of the sun. This is responsible for the heat and light that we get from the sun. On the other hand, a small part of the energy is carried by neutrinos. Because of their small scattering cross sections, the neutrinos come easily out of the sun. Thus, they bring important information a.ivout the solar core.

From Eq. (1.1), one can make a simple estimate for the neutrino flux received on the earth. The total luminosity of the sun is $L_{\odot}=4 \times 10^{33} \mathrm{erg} / \mathrm{s}$. For each $25 \mathrm{MeV}$ energy coming out, two neutrinos are proudced. Thus, the number of neutrinos produced per second is given by $2 L_{\odot} / 25 \mathrm{MeV}$. Dividing this by $4 \pi D^{2}$, where $D$ is the earth-sun distance, $1.5 \times 10^{13} \mathrm{~cm}$, we get for the flux a value of about $6 \times 10^{10} \mathrm{~cm}^{-2} \mathrm{~s}^{-1}$. As seen from Teble 1 , most of this flux is from the $p p$ reaction where deuterium is produced froma two protons.

Table 1 gives the chain of reactions which we summarized in Eq. (1.1). In the first stage, protons synthesize to deuterium $\left({ }^{2} \mathrm{H}\right)$. This occurs in two parallel reactions, called the $p p$ and the pep reactions by the particles that go into them. The $p p$ reastion is responsible for most of the neutrinos produced in the sun. Once deuterium is produced, it quickly synthesizes to ${ }^{3} \mathrm{He}$, and then two ${ }^{3} \mathrm{He}$ can form ${ }^{4} \mathrm{He}$ by strong interaction. However, in very few cases, ${ }^{3} \mathrm{He}$ interacts weakly with protons and produce ${ }^{4} \mathrm{He}$, producing neutrinos in the process.

When some ${ }^{4} \mathrm{He}$ is produced, heavier nuclei like ${ }^{7} \mathrm{Be}$ can be synthesized. Since ${ }^{4} \mathrm{He}$ is a very stable nucleus, the ${ }^{7} \mathrm{Be}$ produced finally turns into ${ }^{4} \mathrm{He}$ through several steps via the production of either ${ }^{7} \mathrm{Li}$ or ${ }^{8} \mathrm{~B}$ as shown in Table 1 . Notice that the neutrino from ${ }^{8} \mathbf{B}$ have very high energy. As we will see later, this is very important for the detection of solar neutrinos. Of course, the Hep neutrinos are also energetic, but their flux iz so small that they can virtually be neglected for our discussion.

There is also the CNO cycle, involving heavier nuclei like various isotopes of carbon, nitrogen and oxygen (hence the name of the cycle) which produces some 
Table 1: Reactions in the pp chain.

\begin{tabular}{|c|c|c|c|}
\hline Reactions & $\begin{array}{c}\text { Name } \\
\text { of reaction }\end{array}$ & $\begin{array}{c}E \\
\text { in } \mathrm{MeV} \\
\end{array}$ & $\begin{array}{c}\text { Flux } \\
\left(10^{10} \mathrm{~cm}^{-2} \mathrm{~s}^{-1}\right) \\
\end{array}$ \\
\hline \multicolumn{4}{|c|}{ Stage 1: $p$ synthesizes to ${ }^{2} \mathrm{H}$} \\
\hline$p+p \rightarrow{ }^{2} \mathrm{H}+e^{+}+\nu_{e}$ & $\mathrm{pp}$ & $\leq 0.42$ & $6.0 \times(1 \pm 0.02)$ \\
\hline$p+e^{-}+p \rightarrow{ }^{2} \mathrm{H}+\nu_{e}$ & pep & 1.44 & $0.014 \times(1 \pm 0.05)$ \\
\hline \multicolumn{4}{|c|}{ Stage $2:{ }^{2} \mathrm{H}$ synthesizes to ${ }^{3} \mathrm{He}$} \\
\hline${ }^{2} \mathrm{H}+p \rightarrow{ }^{3} \mathrm{He}+\gamma$ & - & - & - \\
\hline \multicolumn{4}{|c|}{ Stage $3:{ }^{3} \mathrm{He}$ synthesizes to ${ }^{4} \mathrm{He}$ directly } \\
\hline${ }^{3} \mathrm{He}+{ }^{3} \mathrm{He} \rightarrow{ }^{4} \mathrm{He}+p+p$ & - & - & - \\
\hline${ }^{3} \mathrm{He}+p \rightarrow{ }^{4} \mathrm{He}+e^{+}+\nu_{e}$ & Hep & $\leq 18.77$ & $8 \times 10^{-7}$ \\
\hline \multicolumn{4}{|c|}{ Stage 4: Synthesis of ${ }^{7} \mathrm{Be}$} \\
\hline${ }^{3} \mathrm{He}+{ }^{4} \mathrm{He} \rightarrow{ }^{7} \mathrm{Be}+\gamma$ & - & - & - \\
\hline \multicolumn{4}{|c|}{ Stage 5: ${ }^{7} \mathrm{Be}$ turns into ${ }^{4} \mathrm{He}$} \\
\hline${ }^{7} \mathrm{Be}+e^{-} \rightarrow{ }^{7} \mathrm{Li}+\nu_{e}$ & ${ }^{7} \mathrm{Be}$ & 0.861 & $0.47 \times(1 \pm 0.15)$ \\
\hline${ }^{7} \mathrm{Li}+p \rightarrow{ }^{4} \mathrm{He}+{ }^{4} \mathrm{He}$ & - & - & - \\
\hline${ }^{7} \mathrm{Be}+p \rightarrow{ }^{8} \mathrm{~B}+\gamma$ & - & - & - \\
\hline${ }^{8} \mathrm{~B} \rightarrow{ }^{8} \mathrm{~B}^{*}+e^{+}+\nu_{e}$ & ${ }^{8} \mathrm{~B}$ & $\leq 14.06$ & $5.8 \times 10^{-4}(1 \pm 0.37)$ \\
\hline${ }^{8} \mathrm{~B}^{*} \rightarrow{ }^{4} \mathrm{He}+{ }^{4} \mathrm{He}$ & - & - & - \\
\hline
\end{tabular}

Table 2: The CNO cycle.

\begin{tabular}{|r|c|c|}
\hline Reaction & $\begin{array}{c}E \text { in } \\
\mathrm{MeV}\end{array}$ & $\begin{array}{c}\text { Flux in } \\
10^{10} \mathrm{~cm}^{-2} \mathrm{~s}^{-1}\end{array}$ \\
\hline${ }^{12} \mathrm{C}+p \rightarrow{ }^{13} \mathrm{~N}+\gamma$ & - & \\
${ }^{13} \mathrm{~N} \rightarrow{ }^{13} \mathrm{C}+e^{+}+\nu_{e}$ & $\leq 1.2$ & $0.06(1 \pm 0.50)$ \\
${ }^{13} \mathrm{C}+p \rightarrow{ }^{14} \mathrm{~N}+\gamma$ & - & \\
${ }^{14} \mathrm{~N}+p \rightarrow{ }^{15} \mathrm{O}+\gamma$ & - & \\
${ }^{15} \mathrm{O} \rightarrow{ }^{15} \mathrm{~N}+e^{+}+\nu_{e}$ & $\leq 1.73$ & $0.05(1 \pm 0.58)$ \\
${ }^{15} \mathrm{~N}+p \rightarrow{ }^{12} \mathrm{C}+{ }^{4} \mathrm{He}$ & - & \\
${ }^{15} \mathrm{~N}+p \rightarrow{ }^{16} \mathrm{O}+\gamma$ & - & \\
${ }^{16} \mathrm{O}+p \rightarrow{ }^{17} \mathrm{~F}+\gamma$ & - & \\
${ }^{17} \mathrm{~F} \rightarrow{ }^{17} \mathrm{O}+e^{+}+\nu_{e}$ & $\leq 1.74$ & $5.2 \times 10^{-4}(1 \pm 0.46)$ \\
$p+{ }^{17} \mathrm{O} \rightarrow{ }^{4} \mathrm{He}+{ }^{14} \mathrm{~N}$ & - & \\
\hline
\end{tabular}




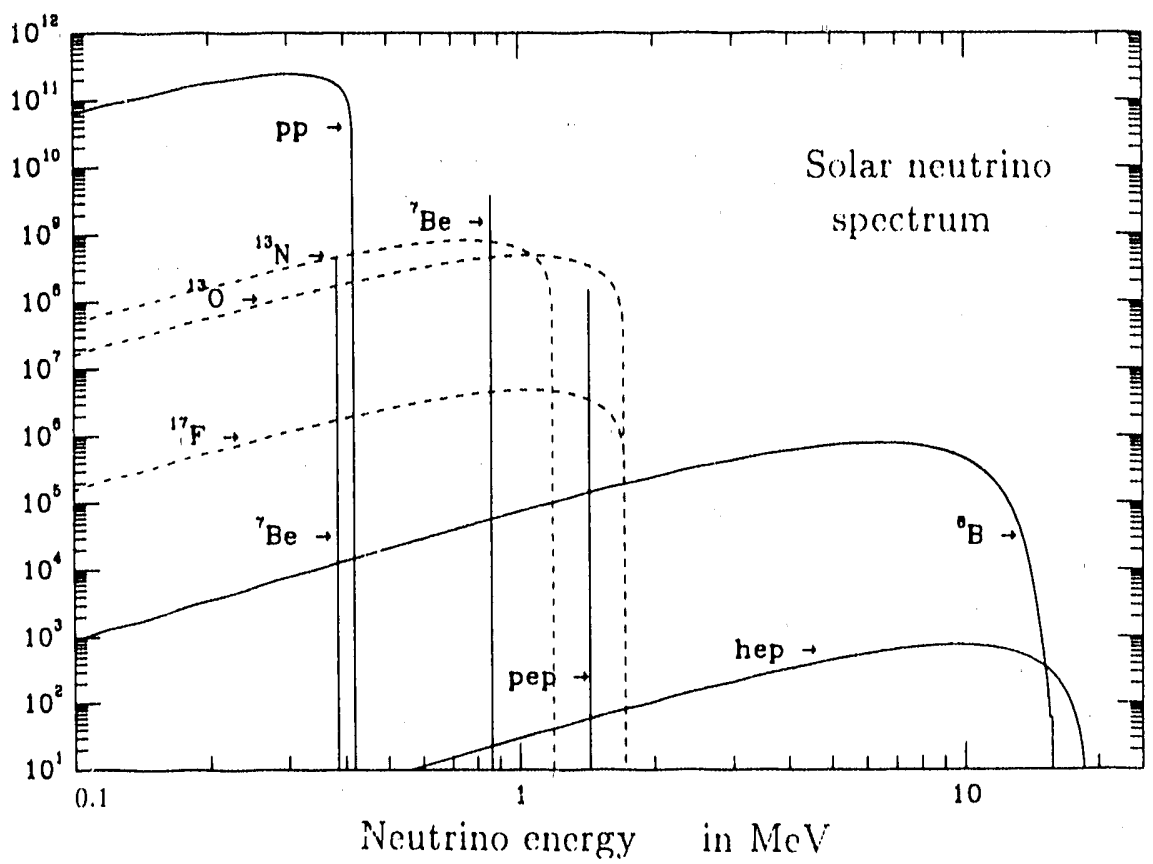

Figure 1: Energy distribution of the flux of solar neutrinos from various reactions. The fluxes from continuum sources are given in the units of number per $\mathrm{cm}^{2}$ per second per $\mathrm{MeV}$ at the mean earth-sun distance. The line fluxes are in number per $\mathrm{cm}^{2}$ per second. Solid lines correspond to the pp chain, and dotted lines to the CNO cycle. Adapted from Ref. [5]. 
neutrinos. The reactions have been shown in Table 2. For the solar core temperature, this cycle is very weak and produces only about $1.5 \%$ of the total neutrino flux.

\section{Detection of solar neutrinos}

\subsection{Principal techniques ${ }^{\dagger}$}

All the detection mechanisms proposed so far for solar neutrinos fall in one of the three categories: (1) Radiochemical, (2) Geochemical, (3) Electron scattering. We discuss the methods briefly ${ }^{\sharp 2}$, summarizing also the results obtained by using them.

Radiochemical detectors : In this method, the $\nu_{e}$ from the sun hits a detector containing some nucleus $X$ which undergoes inverse beta decay:

$$
\nu_{e}+X \rightarrow e^{-}+Y .
$$

The detector is kept active for a while, and then one looks for the resulting nucleus $Y$. The $Y$ nuclei are extracted chemically, and their number gives the neutrino capture rate. The experiments proposed so far involve various target materials, as shown in Table 3. Note that the product nuclei are radioactive. Therefore, one cannot keep on capturing neutrinos for indefinite length of time before trying to detect the $Y$ nuclei chemically.

The advantage of radiochemical detectors is that one can detect low energy neutrinos. The threshold, of course, depends on the material. In ${ }^{71} \mathrm{Ga}$, for example, the threshold is so low that even the low energy $p p$ neutrinos can be detected. The disadvantage is that one cannot tell the times of arrival or the energies of the neutrinos captured.

Geochemical detectors : The basic principle here is the same as that of radiochemical detectors. The difference is that the product nuclei have very long half-life, in the range of $10^{5}$ to $10^{6}$ years. In rock samples or natural ore deposits, one can look for the product nuclei, and their amount will tell us about the solar neutrino flux over the last million years or so. The disadvantage of the method is that one needs a theoretical estimate of how much $Y$ nucleus was supposed to be present primordially in the rock sample. These estimates are not very accurate.

\footnotetext{
"2For details and references, see, e.g. the excellent discussion in Bahcall's book [5].
} 
Table 3: Reactions suitable for radiochemical and geochemical detection of solar $\nu_{e}$ 's. All reactions are of the form $\nu_{e}+X \rightarrow e^{-}+Y$ for suitable nuclui $X$ and $Y$ which are listed.

\begin{tabular}{ccccc}
\hline $\begin{array}{c}\text { Initial } \\
\text { Nucleus }(X)\end{array}$ & $\begin{array}{c}\text { Final } \\
\text { Nucleus }(Y)\end{array}$ & $\begin{array}{c}\text { Threshold } \\
\text { (in MeV) }\end{array}$ & $\begin{array}{c}\text { Half-life } \\
\text { of } Y\end{array}$ & $\begin{array}{c}\text { Capture Rate } \\
\text { (in SNU) }\end{array}$ \\
\hline \hline${ }^{37} \mathrm{Cl}$ & ${ }^{37} \mathrm{Ar}$ & 0.814 & 35 days & $7.9 \pm 2.6$ \\
${ }^{71} \mathrm{Ga}$ & ${ }^{71} \mathrm{Ge}$ & 0.233 & 11.4 days & $132 \pm \pm_{17}^{20}$ \\
${ }^{7} \mathrm{Li}$ & ${ }^{7} \mathrm{Be}$ & 0.862 & 53.4 days & $51.3 \pm 16$ \\
${ }^{127} \mathrm{I}$ & ${ }^{127} \mathrm{Xe}$ & 0.789 & 36 days & $\sim 80$ \\
${ }^{81} \mathrm{Br}$ & ${ }^{81} \mathrm{Kr}$ & 0.470 & $2 \times 10^{5}$ years & $27.8 \pm \pm_{11}^{17}$ \\
${ }^{98} \mathrm{Mo}$ & ${ }^{98} \mathrm{Tc}$ & 1.68 & $4 \times 10^{6}$ years & $17.4 \pm_{11}^{18.5}$ \\
${ }^{205} \mathrm{Tl}$ & ${ }^{205} \mathrm{~Pb}$ & 0.062 & $\sim 10^{7}$ years & $\sim 263$ \\
\hline
\end{tabular}

Electron scattering detectors : In this method, one uses neutrino scattering with electrons:

$$
\nu+e \rightarrow \nu+e
$$

The scattered electron is highly peaked in the forward direction. Thus, by looking at its direction, one can reconstruct the direction of the incoming neutrino and verify that it really came from the sun. This is one big advantage of the method. In addition, one can make an event by event detection, identifying the arrival times and energies of the neutrinos. The disadvantage is that any incident neutral particle can cause the same kind of signature. Thus, one has to subtract the contributions of gamma rays etc. To ensure a good understanding of the background, the threshold for neutrinos has to be kept high.

\subsection{Active experiments and their results}

\subsubsection{The ${ }^{37} \mathrm{Cl}$ experiment}

For more than two decades; Davis and co-workers have been detecting solar neutinos deep under a mine in South Dakota, USA. Their detector contains $2.2 \times 10^{30}$ atoms in the form of liquid $\mathrm{C}_{2} \mathrm{Cl}_{4}$. From Table 3 , notice that the threshold energy is $0.814 \mathrm{MeV}$. Thus, the experiment cannot detect any $p p$ neutrino. Since the absorption cross section rises sharply with energy, the ${ }^{8} \mathrm{~B}$ neutrinos contribute most to the 

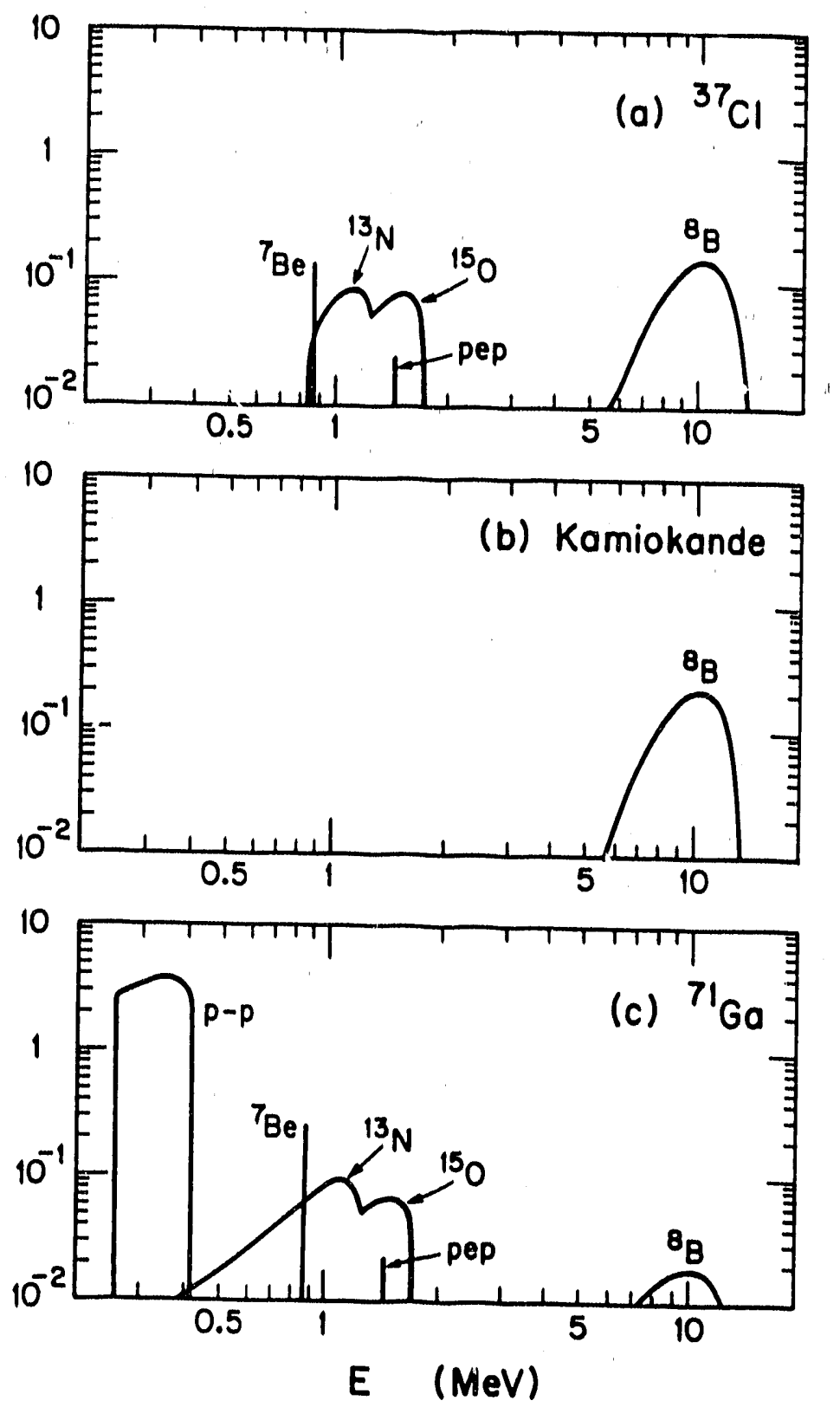

Figure 2: The energy distribution of neutrinos captured in various detectors. Adapted from Ref. [6]. The line sources are given as a fraction of the total signal, and the continuum lines are normalized so that the integrated number is 1 . 


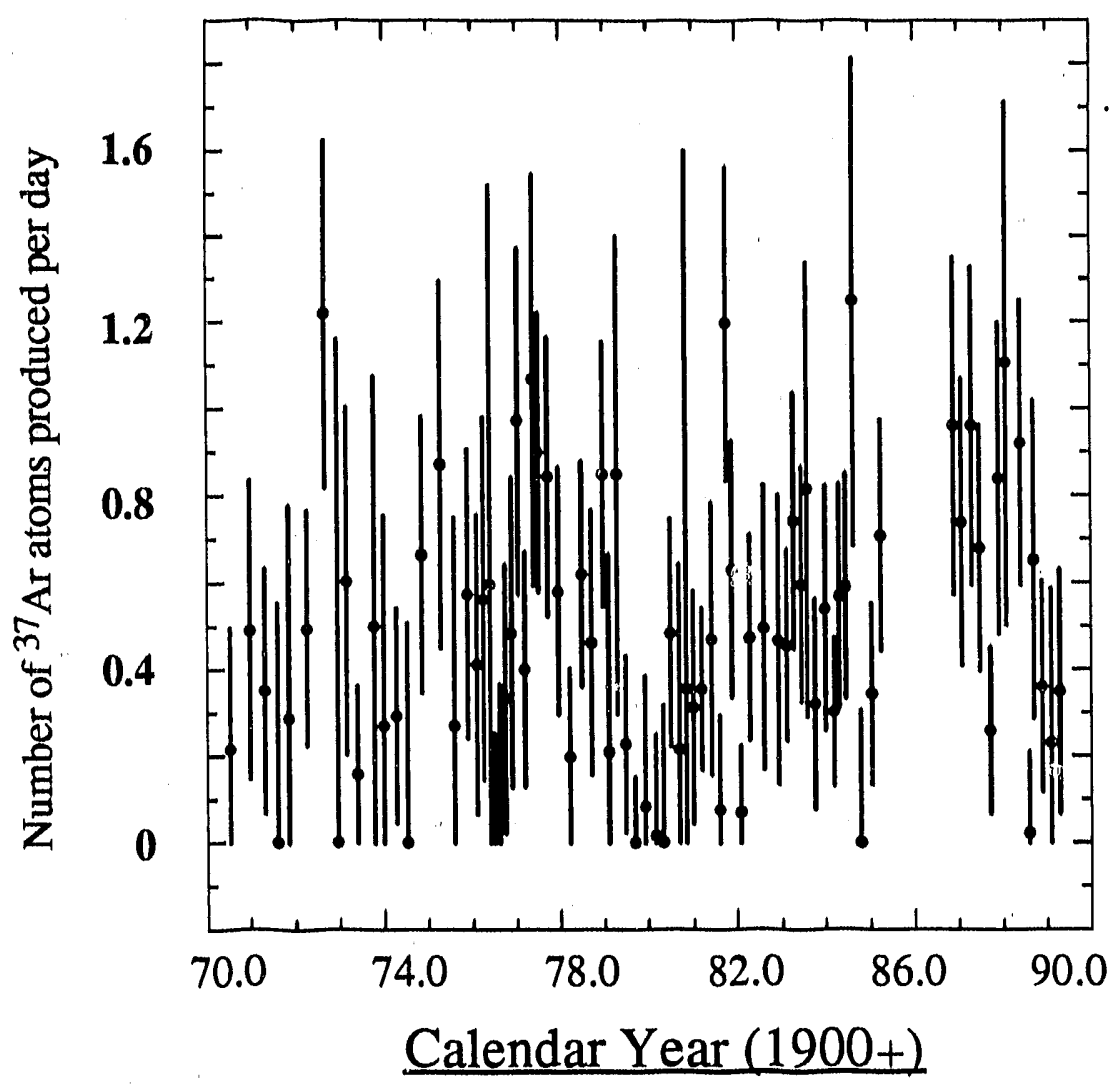

Figure 3: The Davis data.

capture rate. In Fig. 2, we have shown the contribution of neutrinos produced in different reactions to the capture rate. Theoretical calculations with the standard solar model yield the following expectations for the total capture rate [3]:

$$
\phi_{\text {theo }}=(7.09 \pm 0.9) \mathrm{SNU} .
$$

We have put $1 \sigma$ error bars in the number. The unit SNU is defined to be 1 capture per $10^{36}$ target atom per second. Thus, in their detector, production of a single ${ }^{37} \mathrm{Ar}$ atom per day will correspond to a rate of $5.3 \mathrm{SNU}$.

The data obtained by Davis' group has been shown in Fig. 3. The mean value of this data with $1 \sigma$ errors are [7]:

$$
\phi_{\text {Davis }}=(2.1 \pm 0.3) \mathrm{SNU} .
$$

This means

$$
P_{\text {Davis }} \equiv \frac{\phi_{\text {Davis }}}{\phi_{\text {theo }}}=0.27 \pm 0.04
$$

The solar neutrino problem is the statement that $P_{\text {Davis }} \neq 1$. 


\subsubsection{The Kamiokande experiment}

The Kamiokande II detector has been operational since 1986. The energy response for the capture rate is shown in Fig. 2b. Since it detects neutrinos by electron scattering, a high threshold has to be set to allow an event. In the early runs, this was set at $9.3 \mathrm{MeV}$. Later, the background calculations were improved and the threshold was brought down to $7.5 \mathrm{MeV}$. The integrated result from the runs analyzed so far gives [8]:

$$
P_{\text {Kam }}=\frac{\phi_{\text {Kam }}}{\phi_{\text {theo }}}=0.46 \pm 0.05 \text { (stat) } \pm 0.06 \text { (syst). }
$$

Thus, the solar neutrino problem is present in this experiment independent of the Davis result.

\subsubsection{The ${ }^{71} \mathrm{Ga}$, experiment}

The low threshold of Gallium makes it a nice detector, since such a detector can capture many of the neutrinos from the pp reaction. The expected rate is very high. The SAGE group is performing this experiment. So far, they have not found any positive result [9]. Another detector, GALLEX [10] is coming into operation soon.

\section{Part II}

\section{Neutrino oscillation}

The basic idea involved in neutrino oscillation can be explained by its analogy with the more familiar example of spin precession in a transverse magnetic field. Suppose one produces particles of spin $\frac{1}{2}$ whose spins are polarized in the $+z$ (or 'up') direction. The beam travels through a region where there is a magnetic field in the $y$ direction. The 'up' spin is not an eigenstate in this magnetic field. For this, the beam undergoes precession as it travels. If one looks at the beam after it travels some distance, one finds that the beam is a superposition of up and down spins.

Let us restate the last sertence in a uifferent way. We started from a spin up beam, but after it travels some distance, the probability of finding up spin in the beam is less than unity. In other words, there is a "depletion" of up spins. Neutrino oscillation explains the depletion of solar $\nu_{e}$ 's in a similar fashion, i.e., by postulating that the states which are created or detected are not the eigenstates of propagation. 


\section{Vacuum neutrino oscillations and the solar neutrino problem}

\subsection{Hamiltonian formulation of vacuum neutrino oscilla- tions}

The electron neutrino, $\nu_{e}$, is the state produced in a beta-decay where a position $\left(e^{+}\right)$is also produced. The muon neutrino, $\nu_{\mu}$, is the state produced in $\pi^{+}$deciay alongwith $\mu^{+}$. We will call $\nu_{e}$ and $\nu_{\mu}$ as "flavor states". From these definitions, it is not obvious that these flavor states are physical particles. In general, each of them may be superpositions of different physical particles. In other words, the state produced in a beta-decay might have some probability of being a particle $\nu_{1}$, and some probability of being $\nu_{2}$. We will call these states, $\nu_{1}$ and $\nu_{2}$, as the particle or physical states. We introduce the notation

$$
\nu^{(\mathrm{f})}=\left(\begin{array}{c}
\nu_{e} \\
\nu_{\mu}
\end{array}\right), \quad \nu^{(\mathrm{p})}=\left(\begin{array}{l}
\nu_{1} \\
\nu_{2}
\end{array}\right)
$$

and consider the possibility that $\nu^{(f)} \neq \nu^{(\mathrm{p})}$. We can write

$$
\nu^{(\mathrm{f})}=U \boldsymbol{\nu}^{(\mathrm{p})}
$$

where $U$ is called the mixing matrix. Since by our convention, the states in $\boldsymbol{\nu}^{(\mathrm{f})}$ and $\nu^{(\mathrm{p})}$ are orthonormal, $U$ must be a unitary matrix. In the standard inodel of electroweak physics, all neutrinos are massless and hence degenerate. In this case, the matrix $U$ does not have any physical significance. Thus, by introducing the matrix $U$, we are assuming that the neutrinos are not all massless.

If we include the third generation of fermions in this discussion, $\nu^{(\mathrm{f})}$ should have also the state $\nu_{\tau}$, and therefore $\nu^{(\mathrm{p})}$ must have three physical eigenstates. The mixing matrix $U$ will be a $3 \times 3$ matrix. But we restrict ourselves to two generations for the most part of this article. The reason is: two generations are sufficient for explaining the theoretical ideas involved in the solution of the solar neutrino puzzle, and the third generation just makes all the formulas more complicated. Therefore, we will introduce the ideas with the help of two generations and will discuss the effects of the third generation later.

We now study the time evolution [11] of a neutrino beam which, in general, is a superposition of both $\nu_{e}$ and $\nu_{\mu}$ - or alternatively, of $\nu_{1}$ and $\nu_{2}$. The evolution equation would look particularly simple in the basis $\nu^{(p)}$ :

$$
i \frac{d}{d t} \nu^{(\mathrm{p})}(t)=H \nu^{(\mathrm{p})}(t)
$$


where $H$ is the Hamiltonian which is diagonal in this basis:

$$
H=\left(\begin{array}{cc}
E_{1} & 0 \\
0 & E_{2}
\end{array}\right)
$$

In the solar neutrino problem, we will be dealing with neutrinos whose energies are in the $\mathrm{MeV}$ range. Direct laboratory experiments put the following upper bounds on the masses of $\nu_{e}$ and $\nu_{\mu}$ :

$$
m_{\nu_{e}}<12 \mathrm{eV}, \quad m_{\nu_{e}}<250 \mathrm{keV}
$$

Thus, we will assume $m_{\alpha} \ll E_{\alpha}$ for $\alpha=1$, 2. In this case, one can write

$$
E_{\alpha} \equiv \sqrt{\mathbf{p}^{2}+m_{\alpha}^{2}} \simeq|\mathbf{p}|+\frac{m_{\alpha}^{2}}{2|\mathbf{p}|}
$$

For the same reason, we can use $x$, the distance travelled by the neutrino, instead of the time $t$ as independent variable. The difference between $t$ and $x$ will introduce higher order corrections in $m /|\mathbf{p}|$. Thus, the spatial evolution of the neutrino beam is governed by the Hamiltonian

$$
\begin{aligned}
H & =|\mathbf{p}|+\frac{1}{2|\mathbf{p}|}\left(\begin{array}{cc}
m_{1}^{2} & 0 \\
0 & m_{2}^{2}
\end{array}\right) \\
& =\left(|\mathbf{p}|+\frac{m_{1}^{2}+m_{2}^{2}}{4|\mathbf{p}|}\right)-\frac{\Delta}{4|\mathbf{p}|} \sigma_{3}
\end{aligned}
$$

Here, $\sigma_{3}$ is the diagonal Pauli matrix and

$$
\Delta \equiv m_{2}^{2}-m_{1}^{2}
$$

For future purposes, it is better to write down the evolution equation in the flavor basis. We can do this easily using Eq. (3.2) and remembering that $\nu^{(p)}=U^{\dagger} \nu^{(f)}$, getting

$$
i \frac{d}{d x}\left(U^{\dagger} \nu^{(\mathfrak{f})}\right)=H U^{\dagger} \nu^{(\mathfrak{f})}
$$

which gives the following equation of motion for the flavor states:

$$
i \frac{d}{d x} \nu^{(\mathrm{f})}=U H U^{\dagger} \nu^{(\mathrm{f})}
$$

For two Dirac neutrinos,

$$
U=\left(\begin{array}{cc}
\cos \theta & \sin \theta \\
-\sin \theta & \cos \theta
\end{array}\right)
$$


The Hamiltonian in flavor basis is therefore given by:

$$
\begin{aligned}
H^{\prime} & =U H U^{\dagger} \\
& =|\mathbf{p}|+\frac{m_{1}^{2}+m_{2}^{2}}{4|\mathbf{p}|}+\frac{\Delta}{4|\mathbf{p}|}\left(\begin{array}{cc}
-\cos 2 \theta & \sin 2 \theta \\
\sin 2 \theta & \cos 2 \theta
\end{array}\right) .
\end{aligned}
$$

From this, we can deduce the relation between the diagonalizing angle $\theta$ and the elements of the matrix $H^{\prime}$ :

$$
\tan 2 \theta=\frac{2 H_{12}^{\prime}}{H_{22}^{\prime}-H_{11}^{\prime}}
$$

Since $\boldsymbol{H}^{\prime}$ is independent of $x$, we can formally integrate the equation of motion to obtain the solution

$$
\nu^{(f)}(x)=\exp \left(-i H^{\prime} x\right) \nu^{(f)}(0)
$$

To proceed, we make some simplification in the notation. First, we write $|\mathbf{p}|$ simply as $E$. The energy eigenvalues would always appear with some subscript, so there is no chance of confusion. Second, we notice that if there is a term in $H^{\prime}$ which is proportional to the unit matrix, it gives an overall phase to the solution. Moreover, such a term does not affect the mixing angle, as is seen from Eq. (3.13). Thus, such terms are irrelevant for our purpose asd so we drop them. Effectively then,

$$
H^{\prime}=\frac{\Delta}{4 E}\left(\sigma_{1} \sin 2 \theta-\sigma_{3} \cos 2 \theta\right) .
$$

Thereiore,

$$
\begin{aligned}
\boldsymbol{\nu}^{(f)}(x) & =\exp \left[-\frac{i \Delta}{4 E} x\left(\sigma_{1} \sin 2 \theta-\sigma_{3} \cos 2 \theta\right)\right] \nu^{(f)}(0) \\
& =\left[\cos \frac{\Delta}{4 E} x-i\left(\sigma_{1} \sin 2 \theta-\sigma_{3} \cos 2 \theta\right) \sin \frac{\Delta}{4 E} x\right] \nu^{(f)}(0)
\end{aligned}
$$

Probability of finding a $\nu_{\mu}$ or a $\nu_{e}$ in an initial $\nu_{e}$ beam are given by

$$
\begin{aligned}
& P_{\nu_{e} \nu_{\mu}}(x)=\left|\left\langle\nu_{\mu}(0) \mid \nu_{e}(x)\right\rangle\right|^{2}=\sin ^{2} 2 \theta \sin ^{2}\left(\frac{\Delta}{4|\mathbf{p}|} x\right) \\
& P_{\nu_{e} \nu_{e}}(x)=1-P_{\nu_{e} \nu_{\mu}}(x) .
\end{aligned}
$$

Notice that the probability of finding a $\nu_{e}$ is less than unity in general. Pontecorvo [12] suggested that this might cause the flux depletion observed in solar neutrino experiments. 


\subsection{Confronting the solar neutrino data}

To use Eq. (3.17) to explain the solar neutrino data, we should put for $x$ the earth-sun distance, $1.5 \times 10^{13} \mathrm{~cm}$. If we knew $\theta$ and $\Delta$, we could then calculate the survival probability for neutrinos of any energy $E$. Since any experiment detects a spectrum of energies, we need to integrate over that spectrum to find out the survival probability for the entire beum. Let us use the notation

$$
R_{\Delta} \equiv\left\langle\sin ^{2}\left(\frac{\Delta}{4 E} x\right)\right\rangle,
$$

where the angular brackets indicate energy averaging. Then, for a real experiment, the survival probability is given by

$$
P_{\nu_{e} \nu_{e}}=1-R_{\Delta} \sin ^{2} 2 \theta
$$

The quantity $R_{\Delta}$ is, of course, different from one experiment to another. In Fig. 4, we have shown how it behaves as a function of $\Delta$ for the three experiments now in operation [13].

To get an intuitive feeling for these behaviors, first note that

$$
\frac{\Delta}{4 E}=\left(\frac{\Delta}{1 \mathrm{eV}^{2}}\right)\left(\frac{1 \mathrm{MeV}}{E}\right) \cdot 1.25 \times 10^{-2} \mathrm{~cm}^{-1},
$$

so that, for the earth sun distance,

$$
\frac{\Delta}{4 E} x=\left(\frac{\Delta}{1 \mathrm{eV}^{2}}\right)\left(\frac{1 \mathrm{MeV}}{E}\right) \cdot 1.9 \times 10^{11}
$$

Now consider, e.g., the Chlorine experiment. To start with, disregard the intermediate energy neutrinos coming from ${ }^{7} \mathrm{Br},{ }^{13} \mathrm{~N}$ or ${ }^{15} \mathrm{O}$ decay, or from the pep reaction. For the high energy neutrinos coming from ${ }^{8} \mathrm{~B}$ decay, the energy is around $10 \mathrm{MeV}$. If $\Delta \ll 10^{-10} \mathrm{eV}^{2}$, the quantity $(\Delta / 4 E) x$ becomes so small that we hardly have any oscillation, i.e., $R_{\Delta} \simeq 0$. On the other extreme, if $\Delta \gg 10^{-10} \mathrm{eV}^{2}$, the oscillations are so rapid that the effects from different energies average out and we obtain $R_{\Delta}=\frac{1}{2}$. In the region around $10^{-10} \mathrm{eV}^{2}$ we have to really integrate over the spectrum which gives the larger wave-like shapes in Fig. 4.

If now we include the effect of the intermediate energy neutrinos as well, we obtain these spikes of smaller period in $\Delta$ superimposed on the waves of larger period. Since the energy of intermediate energy neutrinos is around $1 \mathrm{MeV}$, the spikes also average out for $\Delta \gg 10^{-11} \mathrm{eV}^{2}$. This explains why the spikes smooth out towards the right end of Fig. 4 a.

Similarly one can understand the $R_{\Delta}$ values for other experiments as well. Once these are known, one can use Eq. (3.19) to find out the survival probability for given 

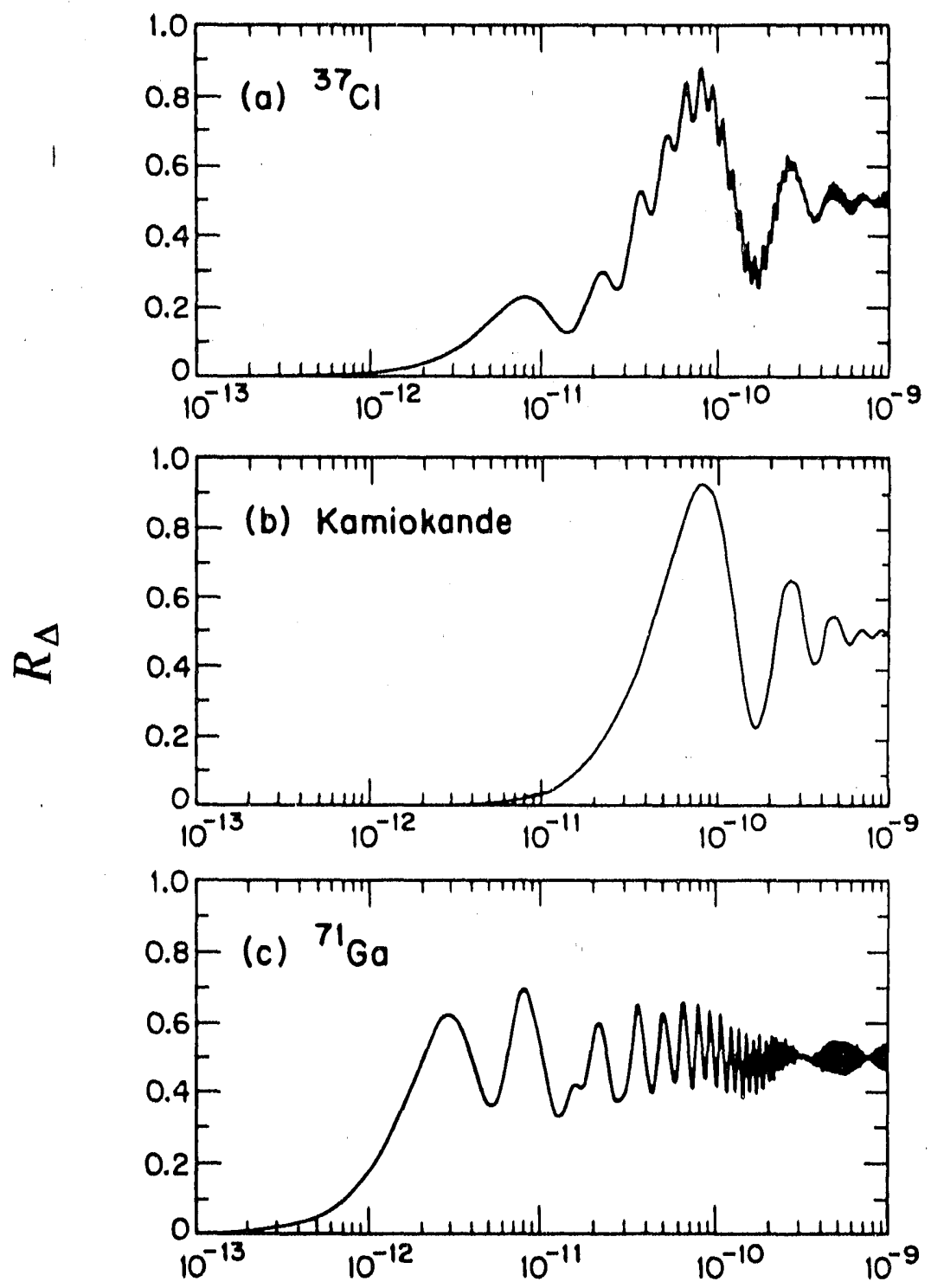

$\Delta$ in $\mathrm{eV}^{2}$

Figure 4: The variation of $R_{\Delta}$ with $\Delta$ for various energy responses. Adapted from Ref. [13]. 
values of $\Delta$ and $\theta$, and see which values are consistent with known experimental data.

For the Chlorine experiment, this is straightforward. From Eq. (2.5), we can say

$$
0.19<P_{\nu_{e} \nu_{e}}<0.35
$$

at the $2 \sigma$ level, i.e., at the $95 \%$ confidence level. For the Kamiokande experiment, there is one important point to remember. The flux that Kamiokande detects is not merely the $\nu_{e}$-flux. If $\nu_{e}$ oscillates to $\nu_{\mu}$ (or $\nu_{\tau}$ ), the $\nu_{\mu}$ will also interact with the detector electrons via neutral current. At the energies relevant for the experiment, $\nu_{\mu} e$ scattering cross section is about one-seventh of the $\nu_{e} e$ cross section. Thus, what Kamiokande detects is

$$
P_{\mathrm{Kam}}=P_{\nu_{e} \nu_{e}}+\frac{1}{7} P_{\nu_{e} \nu_{\mu}}
$$

Since $P_{\nu_{e} \nu_{\mu}}=1-P_{\nu_{e} \nu_{e}}$, one can write

$$
P_{\nu_{\mathrm{e}} \nu_{\mathrm{e}}}=\frac{1}{6}\left(7 P_{\mathrm{Kam}}-1\right)
$$

The result of Eq. (2.6), which gives $P_{\text {Kam }}=0.46 \pm 0.08$ after adding the two errors, gives

$$
0.18<P_{\nu_{e} \nu_{e}}<0.55
$$

at $95 \%$ confidence level. It is this range that has to be compared with the expression in Eq. (3.19).

The plots $[13,14,6]$ of allowed regions in the parameter space of $\Delta$ vs $\theta$ has been shown in Fig. 5. In fact, since it is $\sin ^{2} 2 \theta$ that appears in Eq. (3.19), it is convenient to use $\sin ^{2} 2 \theta$ directly as the $x$-axis variable. As seen from the plot, some small regions of the parameter space are allowed by known data. The region has a very small value of $\Delta$, far smaller than any values explored in terrestrial experiments ${ }^{\sharp 3}$. We will show next that solar matter can enhance neutrino oscillations so that a much larger region of the parameter space becomes acceptable.

\section{Neutrino oscillation in uniform matter}

In the previous discussion, we assumed that the neutrino travels through the vacuum, which is a good approximation to the path between the sun and the earth.

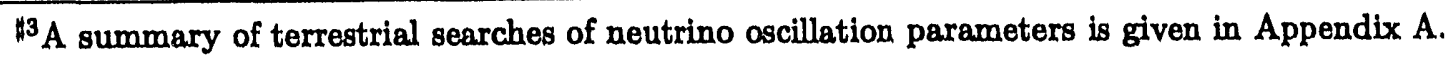




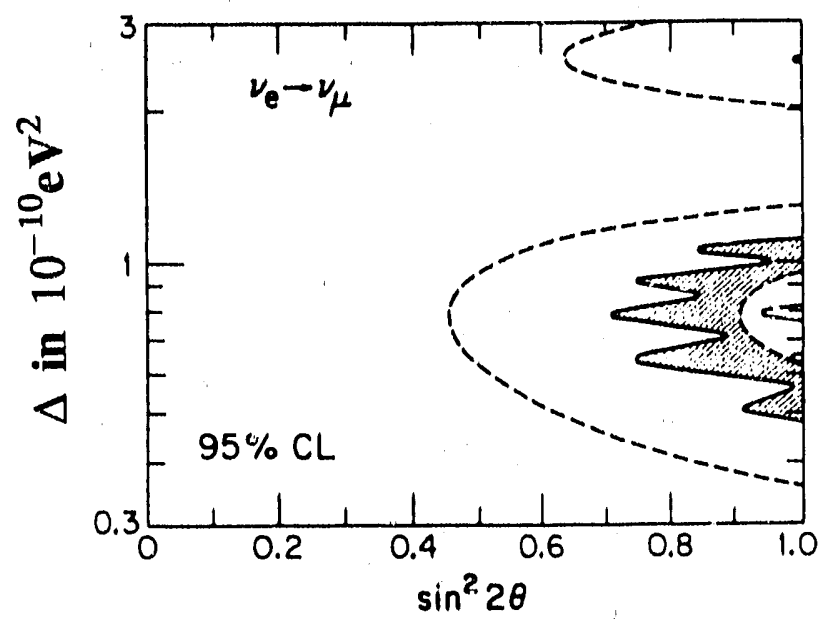

Figure 5: The regions of the parameter space of $\Delta v \operatorname{vin}^{2} 2 \theta$ allowed by both Chlorine and Kamiokande data. The dashed lines correspond to Kamiokande limits and the solid lines to Chlorine experimental limits at $95 \%$ confidence level. Adapted from Ref. [6].

But the neutrinos are produced mostly deep inside the sun, and first they have to pass through the solar material to get out of the sun. Oscillations in the sun, or in any material medium, can be quite different from oscillations in the vacuum. The basic reason for this, as pointed out by Wolfenstein [15], is that interactions in a medium modify the dispersion relation of particles travelling through.

We are very familiar with this phenomenon for the photons. They are massless in the vacuum so that their dispersion relation is simply $E=|\mathbf{p}|$. In a medium, however, the dispersion relation is more complicated, which can be interpreted by saying that the photon develops an effective mass. Because of this, it does not travel with speed $c$ in a medium.

Dispersion relations essentially give the energy of a particle in terms of its momentum. Thus, in quantum mechanical language, a different dispersion relation signifies a different Hamiltonian of the system, which gives a different time evolution of any wave function. For a neutrino beam travelling through the solar material, the evolution is different from that of the same beam travelling in the vacuum.

Solar medium, of course, is non-uniform. Before getting into a discussion of neutrino propagation in such a medium, let us take the simpler case of a neutrino beam travelling through a medium of uniform density. Interactions in the medium affect the dispersion relation of the neutrino. To quantify it, we consider neutrino scattering in matter ${ }^{\sharp 4}$. Solar matter consists of electrons, protons, and neutrons. Of these, the electron neutrino can have charged current interactions with the electron

${ }^{14}$ Here we follow the derivation of Ref. [15]. A field-thoeretical derivation is given in Appendix B. 
only. The $\nu_{\mu}$, on the other hand, could participate in charged current interactions only if muons were present but the solar core temperatures are not high enough for that condition to be satisfied. Hence, so far as the solar interior is concerned, the charged current contributions affect only $\nu_{e}$. The effective Lagrangian for such an interaction is

$$
\begin{aligned}
\mathcal{L}_{\text {eff }} & =\frac{4 G_{F}}{\sqrt{2}}\left\{\bar{e}_{L}\left(p_{1}\right) \gamma^{\lambda} \nu_{e L}\left(p_{2}\right)\right\}\left\{\bar{\nu}_{e L}\left(p_{3}\right) \gamma_{\lambda} e_{L}\left(p_{4}\right)\right\} \\
& =\frac{4 G_{F}}{\sqrt{2}}\left\{\bar{e}_{L}\left(p_{1}\right) \gamma^{\lambda} e_{L}\left(p_{4}\right)\right\}\left\{\bar{\nu}_{e L}\left(p_{3}\right) \gamma_{\lambda} \nu_{e L}\left(p_{2}\right)\right\}
\end{aligned}
$$

where the second form is obtained via Fierz transformation. For forward scattering where $p_{2}=p_{3}=p$, the 4-momentum of the neutrino does not change. Looking at such a case, it would be impossible to know that a scattering phenomenon took place at all. We would rather simply think that the neutrino is propagating with 4-momentum $p$. Therefore, this gives the following contribution to the propagation of the $\nu_{e}$ :

$$
\frac{4 G_{F}}{\sqrt{2}}\left\langle\bar{e} \gamma^{\lambda}\left(\frac{1-\gamma_{5}}{2}\right) e\right\rangle \bar{\nu}_{e L}(p) \gamma_{\lambda} \nu_{e L}(p)
$$

averaging the electron field bilinear over the background.

The solar core temperature is much smaller than the electron mass, so we can consider the electrons to be non-relativistic. It is easy to see, from the explicit forms of the Dirac spinors, that the various averages turn out to be as follows:

$$
\begin{aligned}
\left\langle\bar{e} \gamma_{\lambda} \gamma_{5} e\right\rangle & \sim \text { spin } \\
\left\langle\bar{e} \gamma_{i} e\right\rangle & \sim \text { velocity } \\
\left\langle\bar{e} \gamma_{0} e\right\rangle & =n_{e} .
\end{aligned}
$$

Since spin and velocity are negligible for a collection of non-relativistic particles, the only appreciable contribution to the effective Lagrangian from charged current interactions is given by

$$
\sqrt{2} G_{F} n_{e} \bar{\nu}_{e L} \gamma_{0} \nu_{e L}
$$

Next, we consider neutral current contributions. The effective interaction is

$$
\mathcal{L}_{\text {eff }}=\frac{4 G_{F}}{\sqrt{2}}\left[\bar{f}\left(p_{1}\right) \gamma^{\lambda}\left(I_{3 L} P_{L}-Q \sin ^{2} \theta_{W}\right) f\left(p_{2}\right)\right]\left[\bar{\nu}\left(p_{3}\right) \gamma_{\lambda} P_{L} \nu\left(p_{4}\right)\right]
$$

where $f$ stands for electrons, protons, and neutrons. The symbol $\nu$ can be either $\nu_{e}$ or $\nu_{\mu}$, since both have equal neutral current interactions. Contribution to forward propagation can be determined in exactly the same way as in the case of 
charged current interaction. One obtains the following contribution to the effective Lagrangian:

$$
\sqrt{2} G_{F}\left[\sum_{f} n_{f}\left(I_{3 L}^{f}-2 \sin ^{2} \theta_{W} Q^{f}\right)\right] \bar{\nu}_{L} \gamma_{0} \nu_{L}
$$

In this equation, $I_{3 L}^{f}$ denotes the third component of weak isospin for the fermion $f$, and $Q^{f}$ is the electric charge of the same. For electron, proton and neutron, the values of these quantities are as follows:

\begin{tabular}{c|cc} 
& $I_{3 L}$ & $Q$ \\
\hline $\boldsymbol{e}$ & $-1 / 2$ & -1 \\
$p$ & $1 / 2$ & 1 \\
$n$ & $-1 / 2$ & 0
\end{tabular}

From this, we see that in Eq. (4.6), the electron and proton contributions cancel in a neutral medium where $n_{e}=n_{p}$. The neutral current term is thus given by:

$$
-\frac{1}{\sqrt{2}} G_{F} n_{n}\left(\bar{\nu}_{e L} \gamma_{0} \nu_{e L}+\bar{\nu}_{\mu L} \gamma_{0} \nu_{\mu L}\right) \text {. }
$$

The charged and neutral current contributions, taken together, add terms of the form $\bar{\nu} \gamma^{0} V \nu$ to the effective Lagrangian. The value of $V$ is different for $\nu_{e}$ and $\nu_{\mu}$ :

$$
\begin{aligned}
& V_{\nu_{e}}=\sqrt{2} G_{F}\left(n_{e}-\frac{1}{2} n_{n}\right) \\
& V_{\nu_{\mu}}=-\frac{1}{\sqrt{2}} G_{F} n_{n} .
\end{aligned}
$$

The meaning of such terms is understood if we write down the Dirac equation:

$$
\gamma^{0} E-\boldsymbol{\gamma} \cdot \mathbf{p}-m=\gamma^{0} V .
$$

Rearranging the terms as

$$
\boldsymbol{\gamma}^{0}(E-V)=\boldsymbol{\gamma} \cdot \mathbf{p}+m
$$

and squaring both sides, we finally obtain

$$
E=V+\sqrt{\mathbf{p}^{2}+m^{2}} .
$$

Thus, $V$ just adds to the energy for a given momentum. In this sense, $V$ can be called the potential energy.

Evolution equation in matter is therefore given by

$$
i \frac{d}{d x} \boldsymbol{\nu}^{(\mathrm{f})}=\widetilde{H} \boldsymbol{\nu}^{(\mathrm{f})}
$$


where

$$
\widetilde{H}=H^{\prime}+\left(\begin{array}{cc}
\sqrt{2} G_{F}\left(n_{e}-\frac{1}{2} n_{n}\right) & 0 \\
0 & -\frac{1}{\sqrt{2}} G_{F} n_{n}
\end{array}\right)
$$

$H^{\prime}$ being the vacuum part, given in Eq. (3.12). Thus,

$$
\widetilde{H}=E+\frac{m_{1}^{2}+m_{2}^{2}}{4 E}-\frac{1}{\sqrt{2}} G_{F} n_{n}+\frac{1}{2 E} \widetilde{M}^{2},
$$

where $E$, as before, is a shorthand for the magnitude of the 3-momentum of the neutrino beam, and

$$
\widetilde{M}^{2}=\frac{1}{2}\left(\begin{array}{cc}
-\Delta \cos 2 \theta+2 A & \Delta \sin 2 \theta \\
\Delta \sin 2 \theta & \Delta \cos 2 \theta
\end{array}\right),
$$

where for the sake of convenience, we defined

$$
A=2 \sqrt{2} G_{F} n_{e} E .
$$

The effective mixing angle in matter, $\tilde{\theta}$, would accordingly be given by

$$
\tan 2 \tilde{\theta}=\frac{2 \widetilde{H}_{12}}{\widetilde{H}_{22}-\widetilde{H}_{11}}=\frac{\Delta \sin 2 \theta}{\Delta \cos 2 \theta-A}
$$

and the stationary eigenstates are

$$
\begin{aligned}
& \tilde{\nu}_{1}=\nu_{e} \cos \tilde{\theta}-\nu_{\mu} \sin \tilde{\theta} \\
& \tilde{\nu}_{4}=\nu_{e} \sin \tilde{\theta}+\nu_{\mu} \cos \tilde{\theta}
\end{aligned}
$$

Notice an interesting feature of the eigenstates. As an example, consider that the vacuum mixing angle $\theta$ is small. Then, for $n_{e} \rightarrow 0, \tilde{\theta} \rightarrow \theta$, so that $\tilde{\nu}_{1} \simeq \nu_{e}$. On the other hand for $n_{e} \rightarrow \infty, \tilde{\theta} \rightarrow \frac{\pi}{2}$, so that $\widetilde{\nu}_{1} \simeq \nu_{\mu}$. In other words, the lower mass eigenstate is almost purely $\nu_{e}$ if matter density is vanishing, and is almost purely $\nu_{\mu}$ if matter density if infinite. As we will see, this fact has very important consequences.

The energy-momentum relation in matter is given by the eigenvalues of the matrix $\widetilde{H}$, which are

$$
\tilde{E}_{\alpha}=E-\frac{1}{\sqrt{2}} G_{F} n_{n}+\frac{\widetilde{m}_{\alpha}^{2}}{2 E}
$$

where

$$
\widetilde{m}_{1,2}^{2}=\frac{1}{2}\left[\left(m_{1}^{2}+m_{2}^{2}+A\right) \mp \sqrt{(\Delta \cos 2 \theta-A)^{2}+\Delta^{2} \sin ^{2} 2 \theta}\right] .
$$




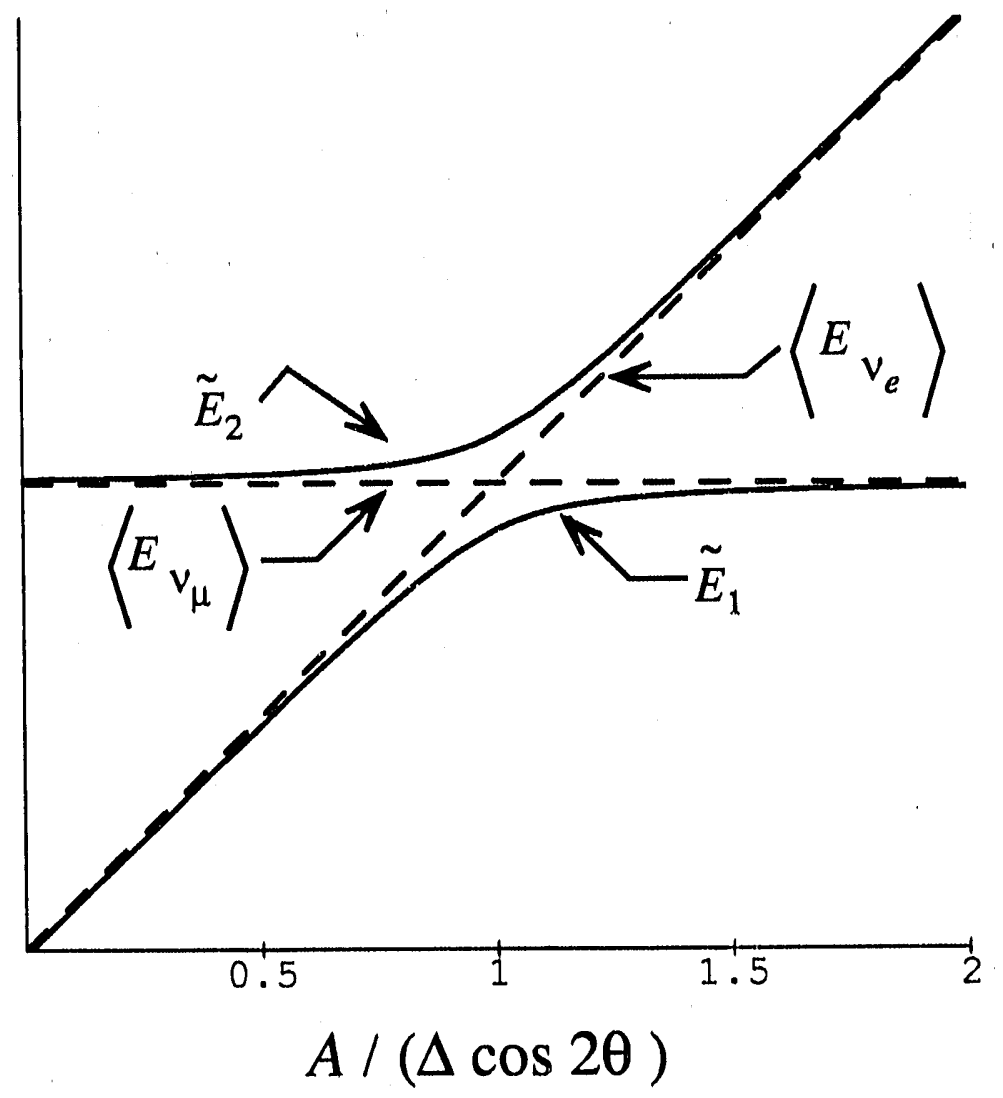

Figure 6: Effective energies of neutrinos in a medium. The quantity $A$ is proportional to the number density of the electrons. The solid lines are the energies for the physical eigenstates, the dashed ones are expectation values of energy for the flavor states. The scale on the vertical axis is arbitrary. We used $\theta=0.1$ for the plot. 
These eigenvalues have been plotted in Fig. 6 as functions of $A$. The figure also shows the diagonal elements of the matrix $\widetilde{H}$, which are the expectation values of energy for the states $\nu_{e}$ and $\nu_{\mu}$. From the figure, it is easily seen that $\tilde{E}_{1}$ is close to $\left\langle\tilde{E}_{\nu_{0}}\right\rangle$ for low densities, whereas it is close to $\left\langle\tilde{E}_{\nu_{\mu}}\right\rangle$ for high densities. This brings out the fact that $\widetilde{\nu}_{1}$ is mainly $\nu_{e}$ at low densities and mainly $\nu_{\mu}$ at high densities. The opposite is true for $\tilde{\nu}_{2}$.

If a medium has a density given by $A=\Delta \cos 2 \theta$, the two diagonal elements are equal. In this case, Eq. (4.18) shows that the effective mixing angle is $\pi / 4$, which is to say that the states $\nu_{e}$ and $\nu_{\mu}$ are maximally mixed in the eigenstates. The nature of this maximal mixing is best understood if, from Eq. (4.18), we write down the expression for $\sin ^{2} 2 \tilde{\theta}$ :

$$
\sin ^{2} 2 \tilde{\theta}=\frac{\Delta^{2} \sin ^{2} 2 \theta}{(\Delta \cos 2 \theta-A)^{2}+\Delta^{2} \sin ^{2} 2 \theta} .
$$

To appreciate the physical significance of the left hand side, we need to look back at Eq. (3.17), which gives the survival and conversion probabilites of a $\nu_{e}$ beam travelling through the vacuum. The corresponding probabilities for a 'eam travelling through a uniform medium can be obtained by replacing $\theta$ by $\tilde{\theta}$ and $\Delta$ by the effective mass difference in the medium. Taking the average of the $x$-dependent term, we obtain

$$
1-\bar{P}_{\nu_{e} \nu_{e}}=\bar{P}_{\nu_{e} \nu_{\mu}}=\frac{1}{2} \sin ^{2} 2 \tilde{\theta}
$$

Thus, the left hand side of Eq. (4.22) is proportional to the conversion probability. The right hand side shows that, as a function of $A$, the conversion probability has the following kind of behavior:

$$
\frac{\text { constant }}{\left(A-A_{R}\right)^{2}+\Gamma^{2}}
$$

This is exactly the expression for a Breit-Wigner resonance of width $\Gamma$ centered at $A_{R}$.

Thus, the import of Eq. (4.22) can be summarized as follows: the conversion probability, as a function of $A$, reaches a resonance at

$$
A_{R}=\Delta \cos 2 \theta
$$

with width

$$
\Gamma=\Delta \sin 2 \theta
$$




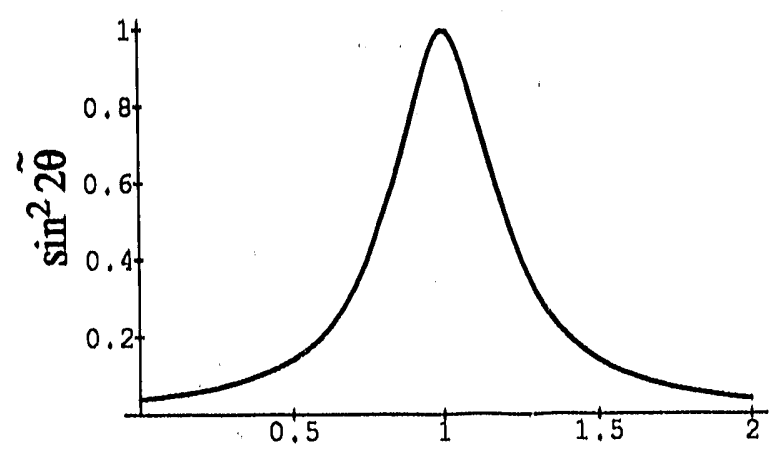

$A /(\Delta \cos 2 \theta)$

Figure 7: The plot of $\sin ^{2} 2 \tilde{\theta}$ shows the resonant shape. We used $\theta=0.1$ for the plot.

Mikheyev and Sminov [16] first realized the importance of the presence of such a resonance in the context of the solar neutrino puzzle. This is the subject we discuss next ${ }^{\sharp 5}$.

\section{Resonant neutrino conversion}

\subsection{Motion of neutrinos in non-uniform matter}

The crucial observation of Mikheyev and Smirnov [16] is that the neutrinos produced deep in the sun will, in general, pass through a region of resonant density on their way out of the sun. To see how this affects neutrino propagation, we first have to derive the evolution equation for matter with non-uniform density, as in the sun. We start with the equation for the flavor states

$$
i \frac{d}{d x} \nu^{(f)}=\frac{1}{2 E} \widetilde{M}^{2} \nu^{(f)} \text {. }
$$

This is the same as Eq. (4.15), except that we omitted the unit matrix terms because they do not affect the probabilities.

But

$$
\boldsymbol{\nu}^{(\mathfrak{f})}=\tilde{U} \tilde{\boldsymbol{\nu}}^{(\mathrm{p})}
$$

where $\tilde{U}$ is a matrix similar to that in Eq. (3.11), with the angle $\theta$ replaced by $\tilde{\theta}$, the effective mixing angle in matter. We can therefore rewrite the evolution equation as:

$$
i \frac{d}{d x}\left(\tilde{U} \widetilde{\nu}^{(\mathrm{p})}\right)=\frac{1}{2 E} \widetilde{M}^{2} \tilde{U} \widetilde{\nu}^{(\mathrm{p})} .
$$

\footnotetext{
"For an earlier review of neutrino oscillation in matter, see Ref. [17].
} 
In taking the derivative, we must remember that, in non-uniform matter, $\tilde{\theta}$ and consequently $\tilde{U}$ are different at different positions. Hence,

$$
i \tilde{U} \frac{d}{d x} \widetilde{\boldsymbol{\nu}}^{(\mathrm{p})}+i\left(\frac{d}{d x} \tilde{U}\right) \tilde{\boldsymbol{\nu}}^{(\mathrm{p})}=\frac{1}{2 E} \widetilde{M}^{2} \tilde{U} \tilde{\boldsymbol{\nu}}^{(\mathrm{p})} .
$$

Using the unitarity of the matrix $\widetilde{U}$, we then obtain the evolution equation for the basis states in $\widetilde{\boldsymbol{\nu}}^{(p)}$ :

$$
i \frac{d}{d x} \widetilde{\boldsymbol{\nu}}^{(\mathrm{p})}=\left(\frac{1}{2 E} \tilde{U}^{\dagger} \widetilde{M}^{2} \tilde{U}-i \widetilde{U}^{\dagger} \frac{d}{d x} \tilde{U}\right) \tilde{\boldsymbol{\nu}}^{(\mathrm{p})} .
$$

The term $\widetilde{U}^{\dagger} \widetilde{M}^{2} \tilde{U}$ is obtained for uniform matter as well, and gives, apart from terms proportional to the unit matrix, the instantaneous eigenvalues $\widetilde{m}_{1}^{2}$ and $\widetilde{m}_{2}^{2}$ in the diagonal entries. The other term can be calculated by using the explicit form of $U$ from Eq. (3.11). This gives [18]

$$
i \frac{d}{d x}\left(\begin{array}{c}
\widetilde{\nu}_{1} \\
\widetilde{\nu}_{2}
\end{array}\right)=\left(\begin{array}{rr}
\frac{\widetilde{m}_{1}^{2}}{2 E} & i \frac{d \tilde{\theta}}{d x} \\
-i \frac{d \tilde{\theta}}{d x} & \widetilde{m}_{2}^{2}
\end{array}\right)\left(\begin{array}{c}
\tilde{\nu}_{1} \\
\tilde{\nu}_{2}
\end{array}\right) .
$$

Note that if $d \tilde{\theta} / d x=0, \widetilde{\nu}_{1}$ and $\widetilde{\nu}_{2}$ are stationary eigenstates indeed. This was the result obtained for a uniform medium. For non-uniform medium like that of the sun, we will have to solve this equation to find different probabilities.

\subsection{Adiabatic solution}

In one situation, the solution of Eq. (5.6) is easy to find. This is the case when $d \tilde{\theta} / d x$ is small, so that we can use the adiabatic approximation. Later, we will quantify how small $d \tilde{\theta} / d x$ has to be for this. Here, we will assume that, the relevant condition is satisfied, so that adiabatic condition prevails $[19,18]$. In that case, $\widetilde{\nu}_{1}$ traverses as $\tilde{\nu}_{1}$, although this means a different superposition of $\nu_{e}$ and $\nu_{\mu}$ at different points. The same is true for $\tilde{\nu}_{2}$. This can suppress the flux.

Consider an illustrative example. Suppose $A_{0} \rightarrow \infty$ (i.e., $n_{e} \rightarrow \infty$ ), where the subscript ' 0 ', from now on, will indicate the quantities at the point where the neutrino is created. From Eq. (4.18) and Eq. (4.19), we then get

$$
\tilde{\theta} \rightarrow \frac{\pi}{2}, \quad \text { i.e., } \quad \nu_{e} \simeq \widetilde{\nu}_{2} .
$$

This $\widetilde{\nu}_{2}$ beam traverses outside the sun, where $A=0$. There,

$$
\nu_{2}=\nu_{e} \sin \theta+\nu_{\mu} \cos \theta .
$$


Probability of finding $\nu_{e}$ in this beam is given by $\left|\left\langle\nu_{e} \mid \nu_{2}\right\rangle\right|^{2}$. Putting the superscript '(ad)' to remind ourselves that we are dealing with the adiabatic solution, we can write

$$
P_{\nu_{e} \nu_{e}}^{(\mathrm{ad})}=\sin ^{2} \theta \text { for } A_{0} \rightarrow \infty
$$

We started with a beam which was almost purely $\nu_{e}$. After it came out of the sun, we see that the probability of finding $\nu_{e}$ in that same beam is $\sin ^{2} \theta$. If $\theta$ is small, this can mean a tremendous suppression. This is the main essence of the resonant neutrino oscillation effect, which is usually called the Mikheyev-SmirnovWolfenstein (MSW) effect by the authors of the pioneering papers $[15,16]$.

In general, at the point of creation, the effective mixing angle is not $\pi / 2$, but has some value $\tilde{\theta}_{0}$. In that case,

$$
\nu_{e}=\widetilde{\nu}_{1} \cos \tilde{\theta}_{0}+\widetilde{\nu}_{2} \sin \tilde{\theta}_{0} .
$$

Thus, there is a probability $\cos ^{2} \tilde{\theta}_{0}$ that the neutrino is produced as $\tilde{\boldsymbol{\nu}}_{: 1}$. If that happens, it travels outside the sun as $\widetilde{\nu}_{1}$ where it can be detected as a $\nu_{e}$ with a probability $\cos ^{2} \theta$. This contributes a term $\cos ^{2} \tilde{\theta}_{0} \cos ^{2} \theta$ to the survival probability $P_{\nu_{e} \nu_{e}}$. Alternatively, the neutrino might be produced as $\tilde{\nu}_{2}$, which has a probability $\sin ^{2} \tilde{\theta}_{0}$. In this case, the probability that it is detected as $\nu_{e}$ is $\sin ^{2} \theta$. Adding these two cases, we then obtain the total survival probability:

$$
\begin{aligned}
P_{\nu_{e} \nu_{\mathrm{s}}}^{(\mathrm{ad})} & =\cos ^{2} \tilde{\theta}_{0} \cos ^{2} \theta+\sin ^{2} \tilde{\theta}_{0} \sin ^{2} \theta \\
& =\frac{1}{4}\left(1+\cos 2 \tilde{\theta}_{0}\right)(1+\cos 2 \theta)+\frac{1}{4}\left(1-\cos 2 \tilde{\theta}_{0}\right)(1-\cos 2 \theta) \\
& =\frac{1}{2}+\frac{1}{2} \cos 2 \tilde{\theta}_{0} \cos 2 \theta .
\end{aligned}
$$

The probability of conversion to $\nu_{\mu}$ is consequently given by

$$
P_{\nu_{e} \nu_{\mu}}^{(\mathrm{ad})}=1-P_{\nu_{e} \nu_{e}}^{(\mathrm{ad})}=\frac{1}{2}-\frac{1}{2} \cos 2 \tilde{\theta}_{0} \cos 2 \theta .
$$

It is worth mentioning here that we have made an implicit assumption in deriving these probabilities. More rigorously, for the detection of $\nu_{e}$ at a distance $x$ from the point of production, we can write

$$
\begin{aligned}
P_{\nu_{e} \nu_{e}}^{(\mathrm{ad})}(x) & =\left|\left\langle\nu_{e}(x) \mid \nu_{e}(0)\right\rangle\right|^{2} \\
& =\left|\sum_{\alpha}\left\langle\nu_{e}(x) \mid \nu_{\alpha}(x)\right\rangle\left\langle\nu_{\alpha}(x) \mid \nu_{\alpha}(0)\right\rangle\left\langle\nu_{\alpha}(0) \mid \nu_{e}(0)\right\rangle\right|^{2},
\end{aligned}
$$

by introducing the complete set of states $\nu_{\alpha}$. The rightmost and the leftmost inner products give the mixing matrix elements at the points of production and detection. 
The middle matrix element gives a phase corresponding to the adiabatic propagation of the states $\nu_{\alpha}$, which is

$$
\exp \left(i \int_{0}^{x} d x^{\prime} \tilde{E}_{\alpha}\left(x^{\prime}\right)\right)
$$

So we get

$$
\begin{aligned}
P_{\nu_{e} \nu_{e}}^{(a d)}(x) & =\left|e^{i \int d x^{\prime} \widetilde{E}_{1}} \cos \tilde{\theta}_{0} \cos \theta+e^{i \int d x^{\prime} \widetilde{E}_{2}} \sin \tilde{\theta}_{0} \sin \theta\right|^{2} \\
& =\frac{1}{2}\left[1+\cos 2 \tilde{\theta}_{0} \cos 2 \theta+\sin 2 \tilde{\theta}_{0} \sin 2 \theta \cos \left(\int_{0}^{x} d x^{\prime}\left(\tilde{E}_{2}-\tilde{E}_{1}\right)\right)\right]
\end{aligned}
$$

The values of $\widetilde{E}_{2}$ and $\widetilde{E}_{1}$ depend on the 3-momentum of the neutrino beam. In Eq. (5.11) and Eq. (5.12), we assumed that the cosine of the energy integral vanishes when one sums over the range of neutrino momenta detected in an experiment. From the discussion in Sec. 3.2 about Fig. 4, this can be seen to imply that $\Delta \gg 10^{-10} \mathrm{eV}^{2}$. As we will see later, the solutions we obtain are consistent with this condition.

\subsection{Non-adiabatic effects}

Non-adiabatic effects induce trarsition between the states $\tilde{\nu}_{1}$ and $\tilde{\nu}_{2}$. Later, we will show that for realistic parameters, such effects are important only around the resonance region [?:0]. For other regions, the adiabatic approximation can be used. In the resonance region, one tries to solve the propagation equation exactly, assuming some simplified forin of density variation that can be approximately valid in that region. From this, one finds the probability that in this region, transition from one eigenstate to another has taken place. We call this probability $X$. Suppose a $\nu_{e}$ produced in the sun was going to survive as a $\nu_{e}$ if the conditions were adiabatic, i.e., if there were no jump between the states $\widetilde{\nu}_{1}$ and $\widetilde{\nu}_{2}$. In the non-adiabatic case, it would still survive as a $\nu_{e}$ provided no jump took place, a phenomenon whose probability is $1-X$. On the other hand, situations that would have ended in a $\nu_{\mu}$ in the adiabatic case, might yield a $\nu_{e}$ in the non-adiabatic case if a jump occurs. Therefore, taking non-adiabatic effects into account, we get [21]

$$
\begin{aligned}
P_{\nu_{e} \nu_{e}} & =(1-X) P_{\nu_{e} \nu_{e}}^{(\mathrm{ad})}+X P_{\nu_{e} \nu_{\mu}}^{(\mathrm{ad})} \\
& =\frac{1}{2}\left[1+(1-2 X) \cos 2 \tilde{\theta}_{0} \cos 2 \theta\right],
\end{aligned}
$$

where the adiabatic probabilities were taken from Eq. (5.11) and Eq. (5.12). Later, we will find $X$ in terms of the fundamental parameters $\Delta$ and $\theta$. Once that is known, Eq. (5.16) gives an analytic formula for determining the survival probability of a $\nu_{e}$ travelling outwards in the sun. 
There is one situation where the expression for the survival probability is somewhat more involved. Consider, for this, a neutrino produced in the far half of the sun. There is a certain probability that will travel towards the earth. If it does, it will pass through the core region. If it is created far enough out in the sun where the density is lower than the resonance density, it crosses the resonance region once while coming towards the core. After that, it crosses the resonance region again on its way out. We can use reasoning similar to that in the previous case to find the survival probability in this case [22]. Here, $P_{\nu_{e} \nu_{e}}^{(a d)}$ needs to be multiplied by the probability that the neutrino either performs no jump near both resonance regions, or that it jumps to the other level in the first resonance region but jumps back in the second region. Similarly $P_{\nu_{e} \nu_{\mu}}^{(a d)}$ will be multiplied by the probability that the neutrino jumps near one resonance region but does not jump near the other one. Thus,

$$
\begin{aligned}
P_{\nu_{e} \nu_{e}} & =\left[(1-X)^{2}+X^{2}\right] P_{\nu_{e} \nu_{e}}^{(\mathrm{ad})}+2 X(1-X) P_{\nu_{e} \nu_{\mu}}^{(\mathrm{ad})} \\
& =\frac{1}{2}\left[1+(1-2 X)^{2} \cos 2 \tilde{\theta}_{0} \cos 2 \theta\right] .
\end{aligned}
$$

Thus, Eq. (5.16) and Eq. (5.17) give us analytic expressions for the survival probability in the general case provided we can express $X$ as a function of the parameters of the problem. To proceed further, we need to find what $X$ is. This is what we do next.

\subsection{The adiabaticity parameter}

Recall the form for the hamiltonian matrix in Eq. (5.6). The adiabatic solutions were obtained by assuming $\tilde{\theta}$ to be a slowly varying parameter. Let us now try to quantify this statement, which will also lead to a plausible expression for $X$.

Simply stated, adiabaticity condition means that the off-diagonal terms in the matrix of Eq. (5.6) are much smaller than the diagonal terms. However, in writing the evolution equation, we omitted unit matrix terms at our will. Such terms would change the diagonal elements, but will keep their difference unchanged. Thus, in diagonal terms, the only physically meaningful quantity is the difference. Therefore, the adiabaticity condition is

$$
\left|\frac{d \tilde{\theta}}{d x}\right| \ll \frac{\left|\widetilde{m}_{1}^{2}-\widetilde{m}_{2}^{2}\right|}{2 E} .
$$

Using the expression for $\tilde{\theta}$ in Eq. (4.18), we get

$$
\frac{d \tilde{\theta}}{d x}=\sqrt{2} G_{F} E \cdot \frac{\Delta \sin 2 \theta}{(\Delta \cos 2 \theta-A)^{2}+\Delta^{2} \sin ^{2} 2 \theta} \cdot \frac{d n_{e}}{d x}
$$


The mass-square eigenvalues were given in Eq. (4.21). Using them, we reduce the adiabaticity condition in Eq. (5.18) to the form

$$
\left|\frac{d n_{e}}{d x}\right| \ll \frac{\left[(\Delta \cos 2 \theta-A)^{2}+\Delta^{2} \sin ^{2} 2 \theta\right]^{\frac{3}{2}}}{2 \sqrt{2} G_{F} E^{2} \cdot \Delta \sin 2 \theta} .
$$

Recalling the expression for the effective mixing angle in Eq. (4.22), this can be rewritten as

$$
\gamma(x) \gg 1
$$

where the quantity

$$
\gamma(x) \equiv \frac{(\Delta / E)^{2}}{2 \sqrt{2} G_{F}} \cdot \frac{\sin ^{2} 2 \theta}{\sin ^{3} 2 \tilde{\theta}} \cdot \frac{1}{\left|\frac{d n_{e}}{d x}\right|}
$$

can be called the "adiabaticity parameter". It depends on the position through $\tilde{\theta}$ and $d n_{e} / d x$.

If the density is very high at some point, $\tilde{\theta} \rightarrow \pi / 2$ so that $\gamma$ becomes high. If matter density is vanishing somewhere, $\tilde{\theta} \rightarrow \theta$, so that once again $\gamma$ is large unless $\theta$ itself is close to $\pi / 4$. In Fig. 8, we plot $\gamma(x)$ for various values of $\Delta / E$ and $\theta$, assuming that the density profile of the sun is given by [23]

$$
n_{e}(r)=98.8 n_{\text {avo }} \exp \left(-\frac{a z^{2}}{z+b}\right)
$$

where $z=r / R_{\odot}, a=11.1, b=0.15$, and $n_{\text {avo }}$ is the convenient unit of Avogadro number of particles in $1 \mathrm{~cm}^{3}$ :

$$
n_{\text {avo }}=6.03 \times 10^{23} / \mathrm{cm}^{3} .
$$

This is a very good fit, as can be seen from Fig. 9.

From Eq. (5.22), it is obvious that the adiabaticity condition is hardest to satisfy at the resonance point, since $\sin 2 \tilde{\theta}$ is maximum there. In fact, in all cases shown in Fig. 8, the regions far from the resonance point can always be treated adiabatically [20]. The jump probability $X$ introduced earlier thus depends only on the conditions near the resonance. Let us call by the symbol $\gamma_{R}$ the value of $\gamma(x)$ at resonance. It can be obtained from Eq. (5.22) by putting $\sin 2 \tilde{\theta}=1$ and can be simplified by using the expression for resonant density

$$
n_{R}=\frac{\Delta \cos 2 \theta}{2 \sqrt{2} G_{F} E}
$$




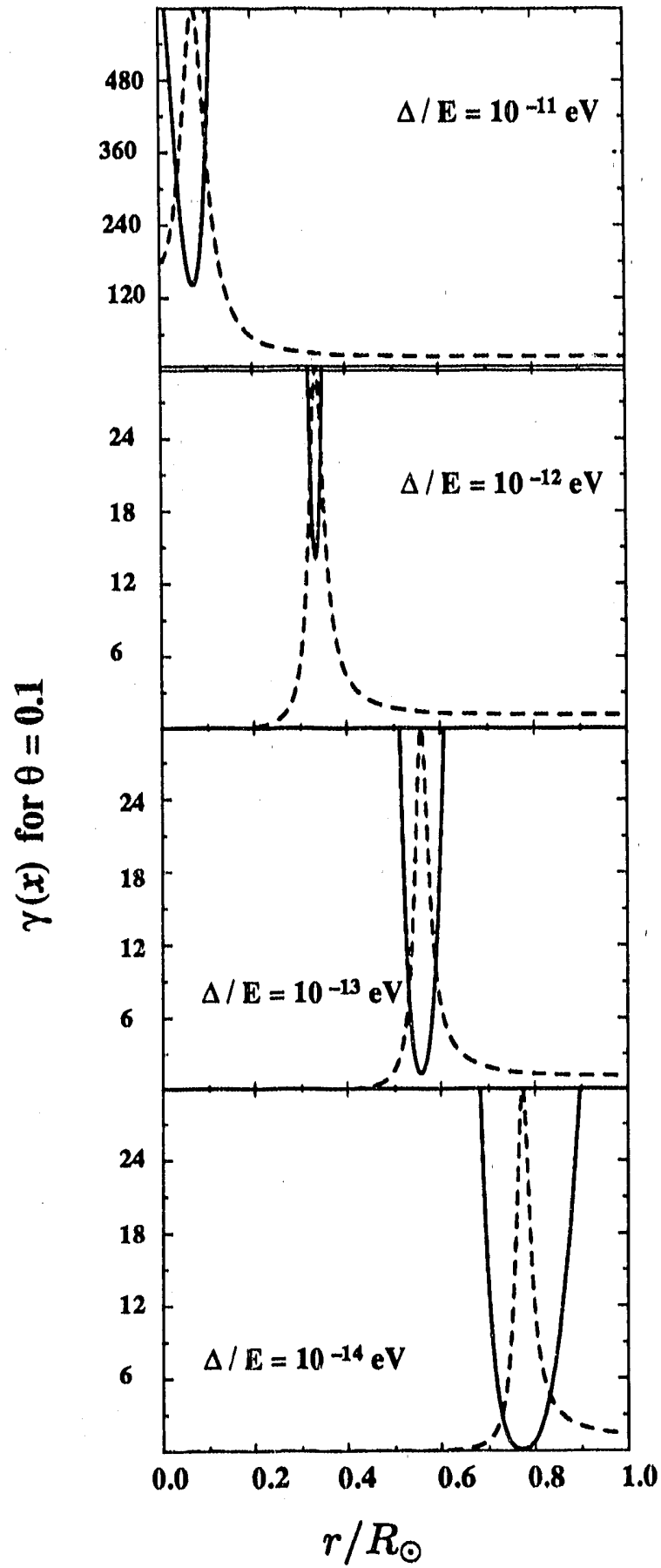

Figure 8: The solid lines give the adiabaticity parameter $\gamma(x)$ as a function of the distance from the solar core, for various values of the parameter $\Delta / E$. The vacuum mixing angle $\theta$ has been taken to be 0.1 . The height of dashed lines are proportional to the values of $\sin ^{2} 2 \tilde{\theta}$ for a neutrino produced at the solar core, with the peak indicating the resonance point. Note different scale for the top graph. 


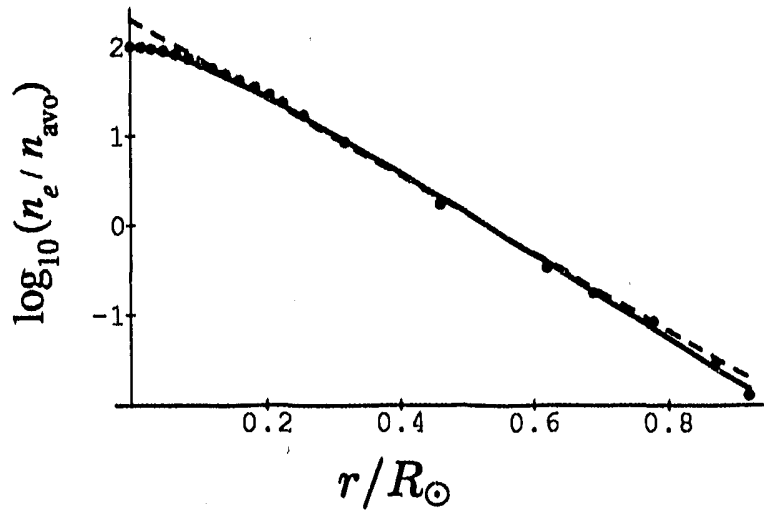

Figure 9: The dots are obtained from solar model calculations. The solid line is the fit from Eq. (5.23). The dashed line is the fit of Eq. (C.1).

which follows from Eq. (4.25) and the definition of $A$. Thus, we obtain

$$
\gamma_{R} \equiv \frac{\Delta}{E} \cdot \frac{\sin ^{2} 2 \theta}{\cos 2 \theta} \cdot \frac{1}{\left|\frac{d}{d x} \ln n_{e}\right|_{R}} .
$$

Of course, if $\gamma_{R} \gg 1$, the propagation is adiabatic everywhere. If, on the other hand, $\gamma_{R}$ is close to or smaller than unity, $X$ is appreciable.

Intuitively, it is clear that $X$ should be larger for higher values of $E$, which means that more energetic neutrinos should jump more readily. As $E \rightarrow 0, X$ should vanish. Also, $X$ cannot be a polynomial in $E$ since the level crossing is essentially a non-perturbative effect. These considerations indicate a solution of the form

$$
X=\exp \left(-\gamma_{R} F\right)
$$

where the quantity $F$ is independent of $E$ and depends on how $n_{e}$ varies with $x$ near resonance. If the variation is linear, for example, $F$ is a constant, as we show in the next section where we outline the derivation of the expression for $X$.

\subsection{Evaluation of the jumping probability ${ }^{\dagger}$}

\subsubsection{The semi-classical approach}

The non-adiabatic transition probability between two states was calculated independently by Landau [24], Zener [25] and Stückelberg [26] in the context of atomic physics problems, and was applied to the present case by Parke [21] and by Haxton [27]. To find the leading behavior in the semiclassical approximation, we can use Landau's method of complex trajectory [28]. This method gives

$$
\ln X=-2 \operatorname{Im}\left[S_{1}\left(t_{1}, t_{*}\right)+S_{2}\left(t_{*}, t_{2}\right)\right],
$$


where $S_{1}\left(t_{1}, t_{*}\right)$, for example, denotes the action for the motion of the neutrino beam in state $\widetilde{\nu}_{1}$ from some initial time $t_{1}$ to a transition time $t_{*}$ which will be specified shortly. After the time $t_{*}$, une uses the action in state $\widetilde{\nu}_{2}$ upto some final time $t_{2}$ when the neutrino beam goes out of the non-adiabatic region. The imaginary parts of the action remains unaffected if we take $t_{1}=t_{2}=t_{R}, t_{R}$ being the time at which the neutrino beam crosses the resonance point. Thus,

$$
\ln X=-2 \operatorname{Im} \int_{t_{R}}^{t_{*}} d t\left(\tilde{E}_{2}-\tilde{E}_{1}\right)
$$

where $\tilde{E}_{\alpha}$ are the energy eigenvalues in the medium, given in Eq. (4.20). We change the integration variable from $t$ to $A$. This gives

$$
\ln X=-\frac{1}{E} \operatorname{Im} \int_{A_{R}}^{A_{*}} \frac{d A}{(d A / d x)} \sqrt{(\Delta \cos 2 \theta-A)^{2}+\Delta^{2} \sin ^{2} 2 \theta},
$$

where we replaced $d A / d t$ by $d A / d x$ since we have been using $t$ and $x$ interchangeably. The lower limit of this integral is $A_{R}=\Delta \cos 2 \theta$. The upper limit, $A_{*}$, is the value of $A$ at the "transition point" mentioned earlier. In Landau's method, this is the value of $A$ for which the two eigenvalues coincide, i.e., $\tilde{E}_{1}=\widetilde{E}_{2}$. From Fig. 6, we see that this does not happen for any real value of $A$. Indeed from Eq. (4.20) and Eq. (4.21) we get

$$
A_{*}=\Delta e^{ \pm 2 i \theta}
$$

which is complex. Thus, the integration of Eq. (5.30) has to be done for complex values of $A$. This is why this method is called the method of complex trajectories.

To evaluate the integral, one needs to know how $A$ behaves as a function of $x$ in the resonance region. If the variation is linear, $d A / d x$ is constant and we can take it outside the integral. If its value is positive, we use the positive exponent in Eq. (5.31) so that $\ln X$ is negative. If $d A / d x$ is negative, we use the negative exponent in Eq. (5.31). In either case, changing the integration variable from $A$ to $a=(A-\Delta \cos 2 \theta) / \Delta \sin 2 \theta$, we get

$$
\ln X=\frac{\Delta^{2} \sin ^{2} 2 \theta}{E|d A / d x|_{R}} \operatorname{Im} \int_{0}^{i} d a \sqrt{1+a^{2}}=\frac{\Delta^{2} \sin ^{2} 2 \theta}{E|d A / d x|_{R}} \cdot \frac{\pi}{4}
$$

Recalling that $A_{R}=\Delta \cos 2 \theta$, we can also write it as

$$
\ln X=\frac{\Delta}{E} \frac{\sin ^{2} 2 \theta}{\cos 2 \theta} \cdot \frac{1}{\left|\frac{d}{d x} \ln A\right|_{R}} \cdot \frac{\pi}{4}
$$

This shows that in Eq. (5.27), we should take $F=\frac{\pi}{4}$, i.e.,

$$
X=\exp \left(-\frac{\pi}{4} \gamma_{R}\right)
$$


The result, of course, depends on the assumption of linear variation of $A$ near the resonace, which might seem a little drastic. For an exponential fall-off in the solar density near the resonance, one obtains [29] $F=\frac{\pi}{4}\left(1-\tan ^{2} \theta\right)$. This is of particular interest since the solar density has indeed an exponential variation for a large region in the sun. However, for most values of interest, the difference between the exponential and the linear case is insignificant. Thus, in what follows, we will stick to the simpler form $F=\frac{\pi}{4}$.

\subsubsection{The extreme non-adiabatic limit}

Landau's method described above is a semiclassical one, and gives the leading term in the limit of large values of the exponent. When $\gamma_{R}$ is very small $(\ll 1)$, the expression for $X$ given in Eq. (5.34) is unsatisfactory.

The limitation of Eq. (5.34) can be understood from a simple example [30]. Consider a neutrino beam travelling through the boundary of a uniform medium into the vacuum. The propagation is obviously adiabatic in the medium as well as in the vacuum, since both have uniform density. But at the boundary, there is an abrupt density change so that $d n_{e} / d x \rightarrow \infty$, which means, through Eq. (5.22), $\gamma \rightarrow 0$, i.e., the situation is highly non-adiabatic. Denoting a point deep inside the medium by $x$ and another far outside by $y$, we can write down the crossing probability $X$ as

$$
\begin{aligned}
X \equiv & \left|\left\langle\nu_{2}(y) \mid \tilde{\nu}_{1}(x)\right\rangle\right|^{2} \\
= & \mid \sum_{\ell}\left\langle\nu_{2}(y) \mid \nu_{2}\left(y^{\prime}\right)\right\rangle\left\langle\nu_{2}\left(y^{\prime}\right) \mid \nu_{\ell}\left(y^{\prime}\right)\right\rangle \\
& \quad \times\left.\left\langle\nu_{\ell}\left(y^{\prime}\right) \mid \nu_{\ell}\left(x^{\prime}\right)\right\rangle\left\langle\nu_{\ell}\left(x^{\prime}\right) \mid \tilde{\nu}_{1}\left(x^{\prime}\right)\right\rangle\left\langle\tilde{\nu}_{1}\left(x^{\prime}\right) \mid \tilde{\nu}_{1}(x)\right\rangle\right|^{2},
\end{aligned}
$$

where $x^{\prime}$ is a point just inside the medium and $y^{\prime}$ is just outside the medium, on two sides of the boundary plane. The flavor states are continuous across the boundary, i.e., $\left\langle\nu_{\ell}\left(y^{\prime}\right) \mid \nu_{\ell}\left(x^{\prime}\right)\right\rangle=1$. Using the mixing matrix in matter and in the vacuum and neglecting all interference terms, we then obtain

$$
X=\sin ^{2}(\tilde{\theta}-\theta) .
$$

If, for example, the medium is very dense, $\tilde{\theta} \rightarrow \pi / 2$ so that

$$
X=\cos ^{2} \theta \text {. }
$$

This is not the limit we obtain from Eq. (5.27), which gives $X \rightarrow 1$ as $\gamma_{R} \rightarrow 0$. The difference is significant if $\theta$ is not small. To convince oneself that it is Eq. 
(5.37) which gives the correct result and not Eq. (5.27), we plug in the expression for $X$ into the expression for survival probability in Eq. (5.16). This gives, using $\tilde{\theta}_{0}=\pi / 2$,

$$
P_{\nu_{e} \nu_{e}}=1-\frac{1}{2} \sin ^{2} 2 \theta
$$

This is the energy-averaged survival probability in the vacuum, as obtained in Eq. (4.23). This is the expected result since, with $\tilde{\theta}=\pi / 2$, there is no oscillation in the medium as can be seen by putting the mixing angel to be equal to $\pi / 2$ in Eq. (3.17). Therefore, oscillation occur only after the beam escapes into the vacuum, and therefore the vacuum result is expected.

Having becoming convinced of the limitation of the exponential form for $X$ in Eq. (5.27), we look for a better formula. This can be obtained by exact solutions of the propagation equation. For this, one usually uses the flavor states directly. Starting from Eq. (4.13), one can write down the two first order differential equations for $\nu_{e}$ and $\nu_{\mu}$. Eliminating $\nu_{\mu}$ from these equations, one gets

$$
\ddot{\nu}_{e}+i\left(\widetilde{H}_{11}+\widetilde{H}_{22}\right) \dot{\nu}_{e}+\left(\widetilde{H}_{12}^{2}-\widetilde{H}_{11} \widetilde{H}_{22}+i \dot{\widetilde{H}}_{11}\right) \nu_{e}=0,
$$

recalling, from Eq. (4.16), that only the $\widetilde{H}_{11}$ element is $x$-dependent. We now use a new variable

$$
a_{e}=\exp \left(i \int^{x} d x^{\prime} \widetilde{H}_{11}\left(x^{\prime}\right)\right) \nu_{e},
$$

which differs from the old variable only by a phase and therefore does not affect the probabilities. In terms of this new variable, the equation becomes $[22,31]$

$$
\ddot{a}_{e}+i\left(\widetilde{H}_{22}-\widetilde{H}_{11}\right) \dot{a}_{e}+\widetilde{H}_{12}^{2} a_{e}=0 .
$$

This equation needs to be solved, putting in the $x$-dependence of $\widetilde{H}_{11}$. Once this is done, the survival probability at a distance $x$ is given by $\left|a_{e}(x)\right|^{2}$.

In principle, if Eq. (5.41) could be solved for the density profile of the sun, one could obtain an exact answer for the survival probabilities of solar neutrinos. This, however, cannot be done because the solar density profile has a complicated shape. So, one solves Eq. (5.41) only near the resonance region and finds the jump probability $X$ exactly, which is given by $1-\left|a_{e}\right|^{2}$. Once this is done, one can use Eq. (5.16) or Eq. (5.17) to find the survival probability after the entire journey through the sun.

The exact solution of Eq. (5.41) was first done for a linear variation of density [21], but since has been performed for a number of other density profiles $[32,33,34$, 
$35,36,30]$. All of these solutions give the form

$$
X=\frac{\exp \left(-\gamma_{R} F\right)-\exp \left(-\gamma_{R} F / \sin ^{2} \theta\right)}{1-\exp \left(-\gamma_{R} F / \sin ^{2} \theta^{\prime}\right)},
$$

where $F$ is to be calculated by using Landau's method. Note that for $\gamma_{R} \rightarrow 0$, this gives the correct limit of Eq. (5.37). Since this form works with all known solutions, it has been conjectured [30] that it works irrespective of the nature of the density variation.

\subsection{Solution summary}

Let us, at this point, summarize the solution of the evolution equation of the neutrino beam given in Eq. (5.6). The probability that a $\nu_{e}$ survives as a $\nu_{e}$ is given by

$$
P_{\nu_{\theta} \nu_{\mathrm{e}}}=\frac{1}{2}\left[1+(1-2 X) \cos 2 \tilde{\theta}_{0} \cos 2 \theta\right] \text {, }
$$

where $\tilde{\theta}_{0}$ is the effective mixing angle at the point where the neutrino is produced, given by

$$
\tan 2 \tilde{\theta}_{0}=\frac{\frac{\Delta}{E} \sin 2 \theta}{\frac{\Delta}{E} \cos 2 \theta-2 \sqrt{2} G_{F} n_{0}},
$$

$n_{0}$ being the number density of electrons at the point of production. As for the jumping probability $X$, we will use the simplest expression

$$
X=\exp \left(-\frac{\pi}{4} \cdot \frac{\Delta}{E} \cdot \frac{\sin ^{2} 2 \theta}{\cos 2 \theta} \cdot \frac{1}{\left|\frac{d}{d x} \ln n_{e}\right|_{R}}\right),
$$

which is valid if the density variation is linear near the resonance.

In order to calculate $P_{\nu_{e} \nu_{e}}$ for neutrinos of a given energy, we thus need two kinds of information. First, we need to know the electron density profile of the sun so that we know $n_{0}$ once we know where the neutrino is produced. The profile will also give us the quantity $\left|\frac{d}{d x} \ln n_{e}\right|$ at the resonance point, which appears in the expression for $X$. Secondly, we need the parameters specifying the particle physics aspects of the problem, viz., the mixing angle $\theta$ and the mass square difference $\Delta$.

In keeping with the spirit of the approach taken here, we will assume that we know the density profile very well and will try to see if the observed solar neutrino fluxes tell us something about the particle physics parameters $\theta$ and $\Delta$. 


\section{Resonant neutrino oscillation confronts the so- lar neutrino data}

\subsection{A simplified example}

To get a simple feeling for the solutions, we make two simplifying assumptions as follows.

1. All neutrinos are produced at a point where the electron number density is $98.8 n_{\text {avo }}$, which is the core density according to solar model calculations.

2. At the point of resonance, $\left|\frac{d}{d x} \ln n_{e}\right|=10 R_{\odot}^{-1}$, independent of where the resonance took place in the sun.

The first assumption is reasonable since most neutrinos are produced near the core where the temperature is high. As for the second, we note that the density profile

$$
n_{e}(r)=200 n_{\mathrm{avo}} e^{-10 r / R_{\odot}}
$$

is in good agreement with astrophysical calculations everywhere except the inner $15 \%$ of the radial distance from the solar core, as seen from Fig. 9. Thus, as long as the resonance does not occur deep inside the sun, the second assumptions does not lead to a drastic simplification of the real problem.

We next note that in Eq. (5.43) through Eq. (5.44), the particle physics parrameter $\Delta$ always appears in the combination $\Delta / E$. Thus, to discuss the solutions, it is better to consider $\Delta / E$ as a single parameter. In Fig. 10, we show the nature of variation of the survival probability $P_{\nu_{e} \nu_{e}}$ with $\Delta / E$ for various values of the vacuum mixing angle $\theta$.

The nature of these curves can be summarized as follows. For high values of $\Delta / E$, the probability of survival is high. As $\Delta_{l} / E$ decreases, there comes a point where the probability starts to fall until it reaches a flat basin. It stays there for a while until, for even lower values of $\Delta / E$, it rises again and reaches a plateau.

The key to the understanding of this nature of variation lies in Eq. (5.44) and Eq. (5.45). We said before that at resonance, the effective mixing angle becomes $\pi / 4$. Outside the sun, i.e., in the vacuum, the mixing anyle is $\theta<\pi / 4$. Thus, the neutrinos will undergo the resonar $\theta$ if, at the point of production, the effective mixing angle exceeds $\pi / 4$. In that case, on its way out of the core, it will pass through the point where $\tilde{\theta}=\pi / 4$ and finally will come out with even lower value of the mixing angle, viz., $\theta$. Thus, resonance conversion occurs provided $\widetilde{\theta}_{0}>\pi / 4$, or, 


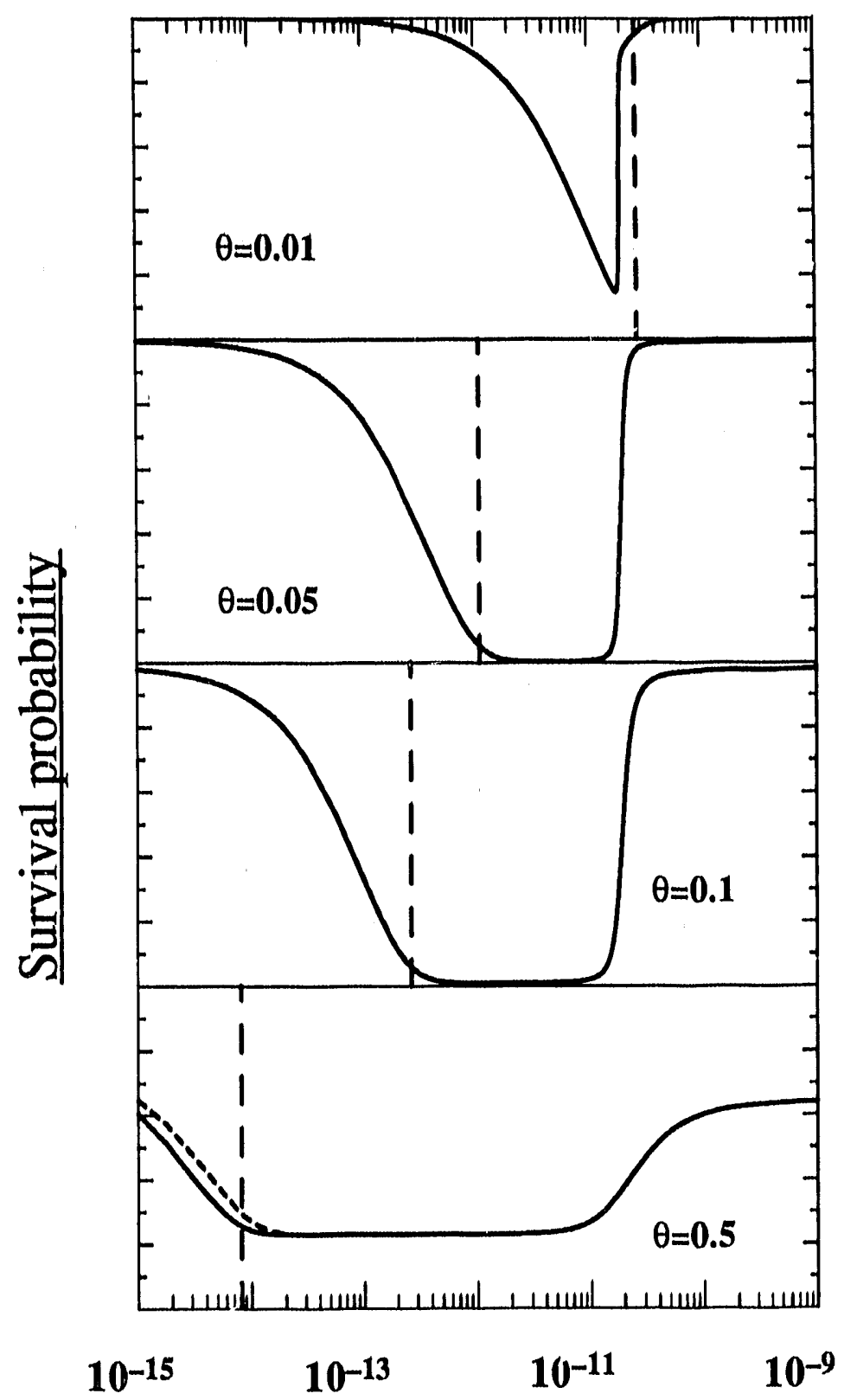

\section{$\Delta / \mathbf{E}$ in $\mathbf{e V}$}

Figure 10: The survival probability as a function of $\Delta / E$ for various values of the vacuum mixing angle $\theta$. The vertical scale runs from 0 to 1 for each graph. The vertical dashed line for each graph corresponds to the value of $\Delta / E$ which gives $X=0.05$. We assumed that the electron number density at the point of neutrino production is $n_{0}=98.8 n_{\text {avo }}$. For $\theta=0.5$, the short dashes indicate the probability obtained by using $F=\frac{\pi}{4}\left(1-\tan ^{2} \theta\right)$ in Eq. (5.27). For other values of $\theta$ shown here, the distinction between this and $F=\frac{\pi}{4}$ is not appeciable on the scale of the plot. 
from Eq. (5.44), if

$$
\frac{\Delta}{E} \cos 2 \theta<2 \sqrt{2} G_{F} n_{0}=1.5 \times 10^{-11} \mathrm{eV} .
$$

The other important point to understand in the curves of Fig. 10 is the importance of non-adiabatic effects. Obviously, such effects are small as long as $X \ll \frac{1}{2}$, as is seen from Eq. (5.43). Let us say the non-adiabatic effects are "important" if $X>0.05$. For a linear variation of density near the resonance, this occurs when $\gamma_{R}<3.8$, where $\gamma_{R}$ has been defined in Eq. (5.26). With our parametrization of the quantity $\left|\frac{d}{d x} \ln n_{e}\right|$, this gives

$$
\frac{\Delta}{E} \cdot \frac{\sin ^{2} 2 \theta}{\cos 2 \theta}<10^{-14} \mathrm{eV},
$$

using $R_{\odot}=7 \times 10^{10} \mathrm{~cm}$. In all the plots of Fig. 10, we have marked the value of $\Delta / E$ corresponding to the equality sign in the last equation. The non-adiabatic effects are important to the left of this mark.

At the right end of the plots of Fig. 10, the value of $\Delta / E$ is so large that neither the resonance condition Eq. (6.2), nor the non-adiabaticity condition, Eq. (6.3), is satisfied. Thus, $X \simeq 0$ and from Eq. (5.44), $\tilde{\theta}_{0} \simeq \theta$, so that $P_{\nu_{\mathrm{e}} \nu_{\mathrm{e}}} \simeq \frac{1}{2}\left(1+\cos ^{2} 2 \theta\right)$, which is the average survival probability in the vacuum. As $\Delta / E$ decreases, at some point it satisfies Eq. (6.2). For values of $\theta \ll 1$, this occurs when $\Delta / E=$ $1.5 \times 10^{-11} \mathrm{eV}$. Around this point, the survival probability drops down because of resonant conversion of $\nu_{e}$ into $\nu_{\mu}$. The range of values of $\Delta / E$ over which this fall takes place, is determined by the width of the resonance given in Eq. (4.26). It is clear from the plots that this width increases with increasing $\theta$, as expected from Eq. (4.26). For $\Delta / E$ substantially smaller than the resonant value the adiabatic survival probability is just $\sin ^{2} \theta$, as argued in Eq. (5.9). This corresponds to the basins in the plots.

If there were no non-adiabatic effects, the survival probability would have stayed in that basin for all lower values of $\Delta / E$. However, at some point depending on the value of $\theta$, non-adiabatic effects become important, which results in a higher probability of survival. The onset of this effect, as shown in Eq. (6.3), occurs for lower and lower values of $\Delta / E$ for higher and higher values of $\theta$. In the plot corresponding to $\theta=0.01$, this onset occurs before the adiabatic basin is reached, so that the probability never goes down to $\sin ^{2} \theta$, in contrast the other cases shown.

We now represent the solutions in a different way which would be more useful later. Consider, for example, neutrinos of a fixed energy, and suppose we know experimentally that their survival probability is between 0.19 and 0.35 . We want to find out which values of $\Delta$ and $\theta$ will be consistent with this range of survival 


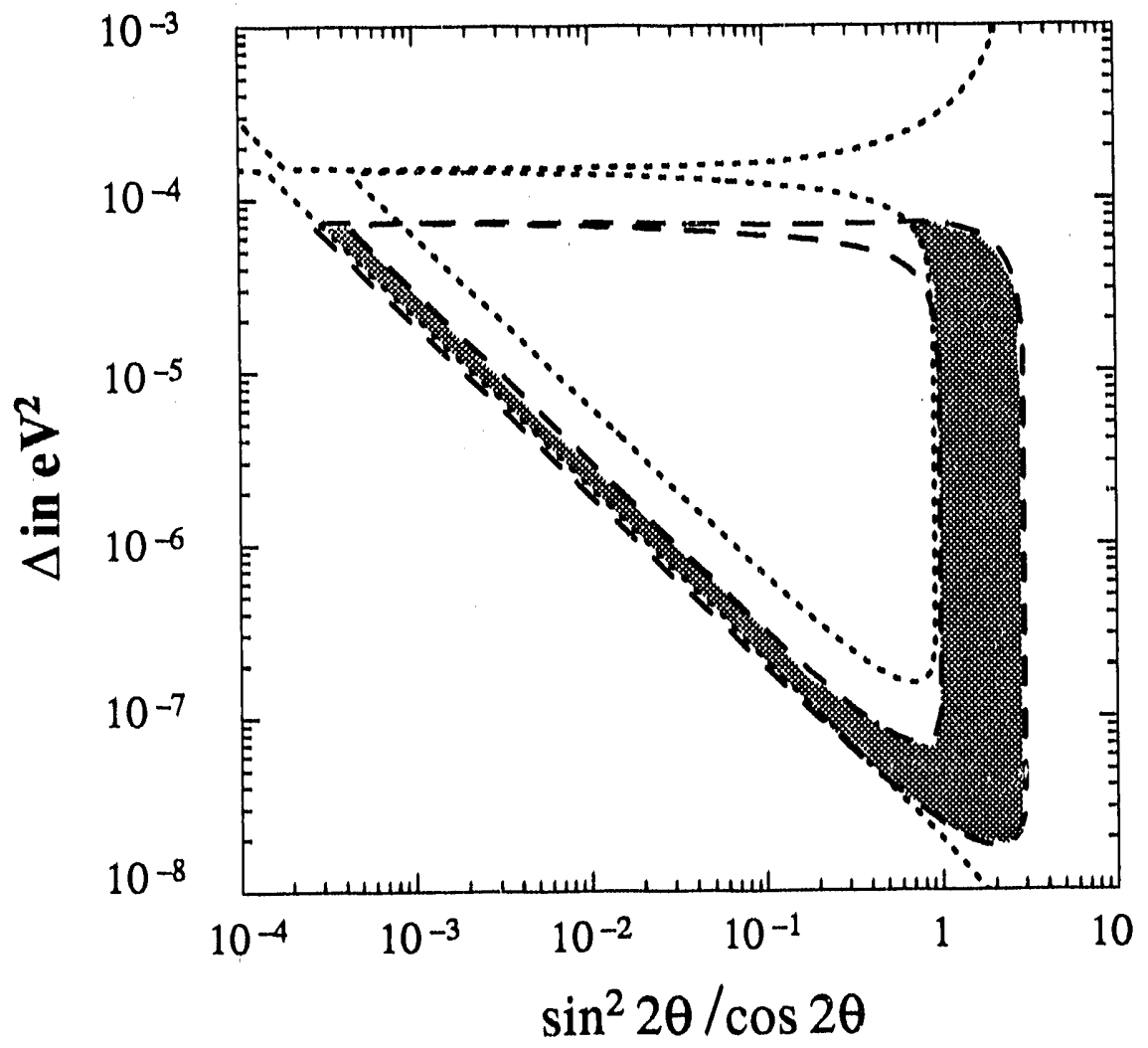

Figure 11: The dashed lines show the allowed region of the parameter space if, for $E=5 \mathrm{MeV}$, one finds $0.19<P_{\nu_{\mathrm{e}} \nu_{\mathrm{e}}}<0.35$. The dotted line is the same for $E=10 \mathrm{MeV}$ if $0.18<P_{\nu_{e} \nu_{e}}<0.55$. The shaded region is allowed by both the conditions. 
probability. For $\theta=0.01$ in Fig. 10, we find an almost continuous region around the lowest point. As we go to the higher value $\theta=0.05$, we find two clearly different regions of solutions - one in the adiabatic region and the other in the non-adiabatic region, separated by the basin where the survival probability is lower than the values specified in the range. As $\theta$ becomes larger, the adiabatic solution occurs still roughly at the same value of $\Delta / E$, but the non-adiabatic solution recedes further owing to a broader basin. The basin, for increasing values of $\theta$, becomes more and more shallow. For $\theta=0.5$, we see that even the whole basin is within the specified range of probabilities, so we obtain a large band of values of $\Delta / E$ for that value of $\theta$. Putting in the value of $E$, we can mark the allowed region in a plot of $\theta$ vs $\Delta$. This plot for $E=5 \mathrm{MeV}$ has been shown in dashed lines in Fig. 11. Note that the horizontal axis of this plot is not $\theta$ but the combination $\sin ^{2} 2 \theta / \cos 2 \theta$ that appears in the expression for $X$. The advantage of using this as the independent variable will be explained later.

As the figure shows, the contour corresponding to a certain survival probability is roughly a triangular region in the plot. The upper and lower limits of the survival probability thus select out a triangular band-shaped region. With just one experiment, this is the best one can do to determine $\Delta$ and $\theta$.

But now suppose we make another experiment with $E=10 \mathrm{MeV}$ and obtain that the survival probability lies between 0.18 and 0.55 . This will similarly correspond to a similar region in the parameter space, which has been shown by a dotted line. Combining this result with the previous one, we can narrow down the range of allowed solutions considerably. For example, the figure shows that the horizontal parts of the solution are eliminated since they do not overlap. The vertical and the diagonal branches are also narrower in the overlap region than in either of the individual plots.

This procedure can be continued if one has more data at different energies. The solution space can be restricted by the overlap of all the data available.

The simple example shown here is very close to the actual calculations done for solar neutrinos. In fact, to make the example realistic, we have chosen the ranges of survival probability corresponding to the dashed and the dotted lines to be equal to the probabilities observed in Chlorine and Kamiokande experiments at the $95 \%$ confidence level. The fact that we took $E=5 \mathrm{MeV}$ for the dashed lines and $E=10 \mathrm{MeV}$ for the dotted ones is reminiscent of the fact that at an average, the Kamiokande detector detects higher energy neutrinos than the Chlorine detector. That is why most of the features of the solution obtained here are similar to those obtained by elaborate analysis, which we discuss next. 


\subsection{The full analysis}

The full calculation of survival probabilities for solar $\nu_{e}$ 's involves two levels of complication which were left out in the simplified example given above. First, the solar neutrino flux is not monochromatic, so one has to integrate over the energy response. For different experiments, these responses were plotted in Fig. 2. Second, in the simpler example we assumed all neutrinos are produced at the center. In the detailed calculation, one has to take account of the distribution of the point of production of the neutrino. We would also like to use the better density profile of Eq. (5.23) rather than Eq. (6.1).

The distribution of production point of neutrinos is obtained directly from the solar model calculations. One important point to remember here is that some neutrinos are created in the far half of the sun, and they undergo level crossing twice. For such neutrinos, one must use Eq. (5.17) to determine the survival probabilities.

Results of numerical integrations $[6,37,38,39]$ over energy and production point have been shown in Fig. 12. Qualitatively, the shape of the parameter space allowed by Chlorine and Kamiokande data is similar to the shape of the allowed regions of Fig. 11. For each experiment, there is a horizontal, a vertical and diagonal region. Let us try to have some intuitive feeling for these branches of solutions.

Resonant conversion occurs provided $\tilde{\theta}_{0}>\pi / 4$, or, from Eq. (5.44), provided

$$
\Delta \cos 2 \theta<A_{0},
$$

where

$$
A_{0}=2 \sqrt{2} G_{F} n_{0} E=1.5 \times 10^{-5} \mathrm{eV}^{2} \cdot\left(\frac{E}{1 \mathrm{MeV}}\right)\left(\frac{n_{0}}{98.8 n_{\text {avo }}}\right) .
$$

Consider now the horizontal branch [19]. As explained in connection with Fig. 10, this branch represents adiabatic neutrino propagation. Since $\theta \ll 1$ for the most part here, we can write the resonance condition as

$$
\Delta<1.5 \times 10^{-5} \mathrm{eV}^{2} \cdot\left(\frac{E}{1 \mathrm{MeV}}\right)\left(\frac{n_{0}}{98.8 n_{\text {avo }}}\right) .
$$

For a given value of $\Delta$, if this condition is satisfied for some value of $E$, all neutrinos with that energy are converted. Only low energy neutrinos survive.

Next, consider the vertical branch. This is also adiabatic. Here the resonance condition is satisfied for almost all relevant energies because either $\Delta$ is small or $\cos 2 \theta$ small, or both. All neutrinos survive with probability $\sin ^{2} \theta$ irrespective of $E$. Allowed range of $\theta$ corresponds to $\sin ^{2} \theta$ being the observed probability. 


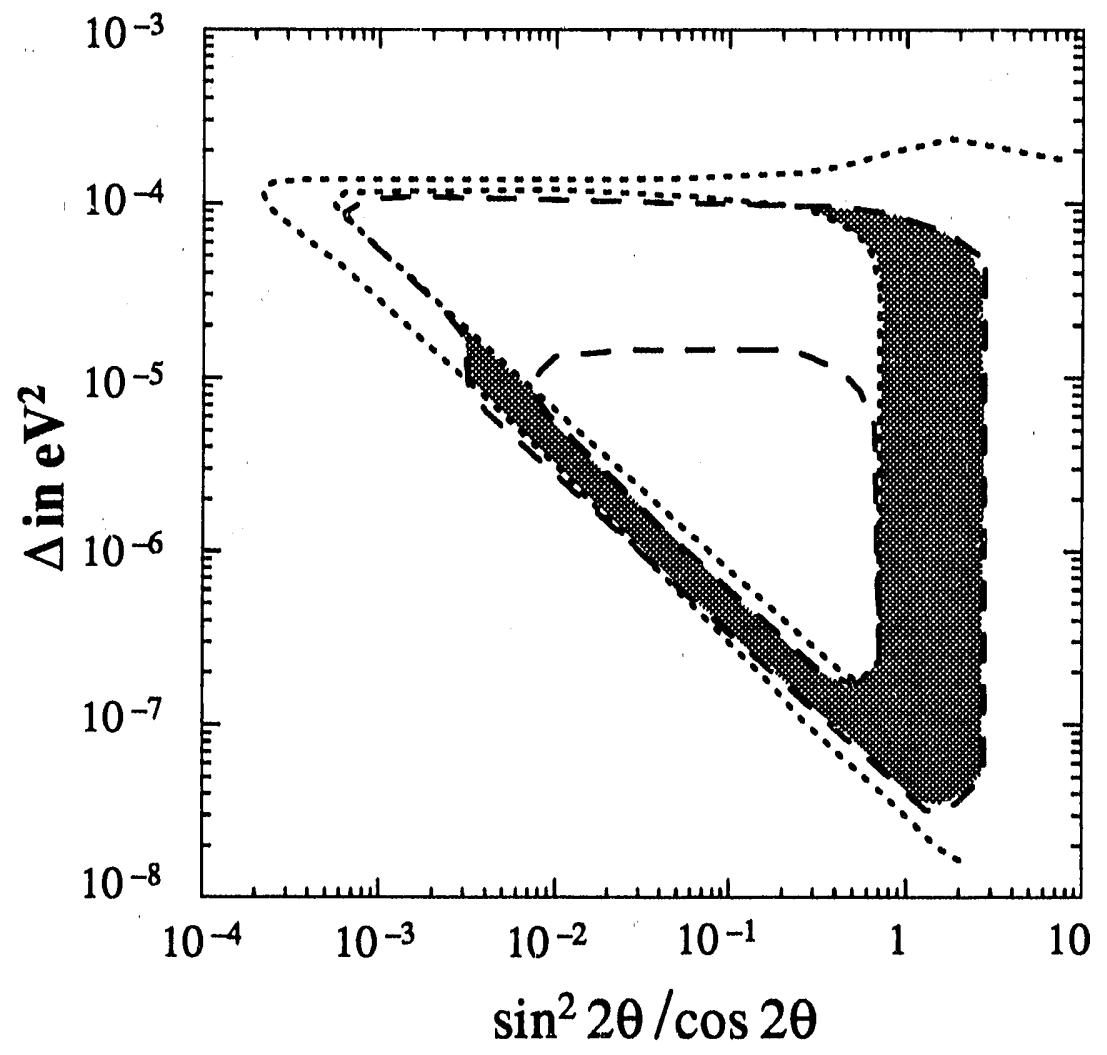

Figure 12: The dashed lines show the allowed region of the parameter space if $0.19<P_{\nu_{e} \nu_{e}}<0.35$ for the energy response of the Chlorine experiment as shown in Fig. 2. The dotted line is the same for $0.18<P_{\nu_{e} \nu_{e}}<0.55$ where the Kamiokande energy response has been used. The shaded region is allowed by both the conditions. 
The diagonal branch $[31,40,41]$ is non-adiabatic. To understand why this portion is almost straight, recall the expression of survival probability from Eq. (5.43). If the density of production is high, $\tilde{\theta}_{0} \simeq \pi / 2$. If, moreover, $\theta \ll 1$, then we obtain $P_{\nu_{e} \nu_{\mathrm{e}}} \simeq X$. Contours of constant $P_{\nu_{\mathrm{e}} \nu_{\mathrm{e}}}$ are then contours of constant $X$ to a good approximation. But from Eq. (5.34), $\log X \propto \gamma_{R}$, so that we need lines for constant $\gamma_{R}$. Recalling the expression for $\gamma_{R}$ in Eq. (5.26), we see that for a fixed energy, equal $P_{\nu_{e} \nu_{e}}$ lines give $\Delta \sin ^{2} 2 \theta / \cos 2 \theta=$ constant. These are diagonal straight lines in a log-log plot if we plot $\Delta$ vs $\sin ^{2} 2 \theta / \cos 2 \theta$. This is the reason for taking this combination as the independent variable in the plot rather than plotting $\theta$ directly. Another advantage of this variable is that it expands the region near $\theta=\pi / 4$, so that the vertical branch solution can be read clearly from the graph.

Note that the energy distributions have different characters for the three branches. In the horizontal branch, the low energy neutrinos survive, the high energy ones are converted. In the vertical branch, the conversion probability is independent of energy. In the non-adiabatic branch, the low energy neutrinos are predominantly converted, high energy ones survive.

From this, it is not hard to see why the horizontal branch solution is ruled out when we take both Davis and Kamiokande results into account [42]. The average energy of detected neutrinos is higher in Kamiokande experiment. If the horizontal branch solution were correct, they should have seen a larger suppression in flux than the Davis experiment. The results, however, do not indicate that.

Once enough data is available to know the energy distribution of the neutrinos detected, one can ascertain from any single experiment which branch does the solution lie in. Since chemical detectors cannot detect the energy of the neutrinos, they are unsuitable for this purpose. Kamiokande can decide this issue with more data. In fact, even with the present data, they argue that they see more depletion in the lower energy sector, so that the horizontal branch is ruled out without any input from other experiments [43]. The situation will be more clear when they have more statistics.

\section{Variations on the theme ${ }^{\dagger}$}

\subsection{What if $\nu_{e}$ oscillates to a sterile neutrino?}

So far, we have assumed that $\nu_{e}$ oscillates to $\nu_{\mu}$. This assumption is not supported by any experiment. All we know, from Davis as well as Kamiokande experiments, is that the $\nu_{e}$ 's are converted to something - but we do not know what. 

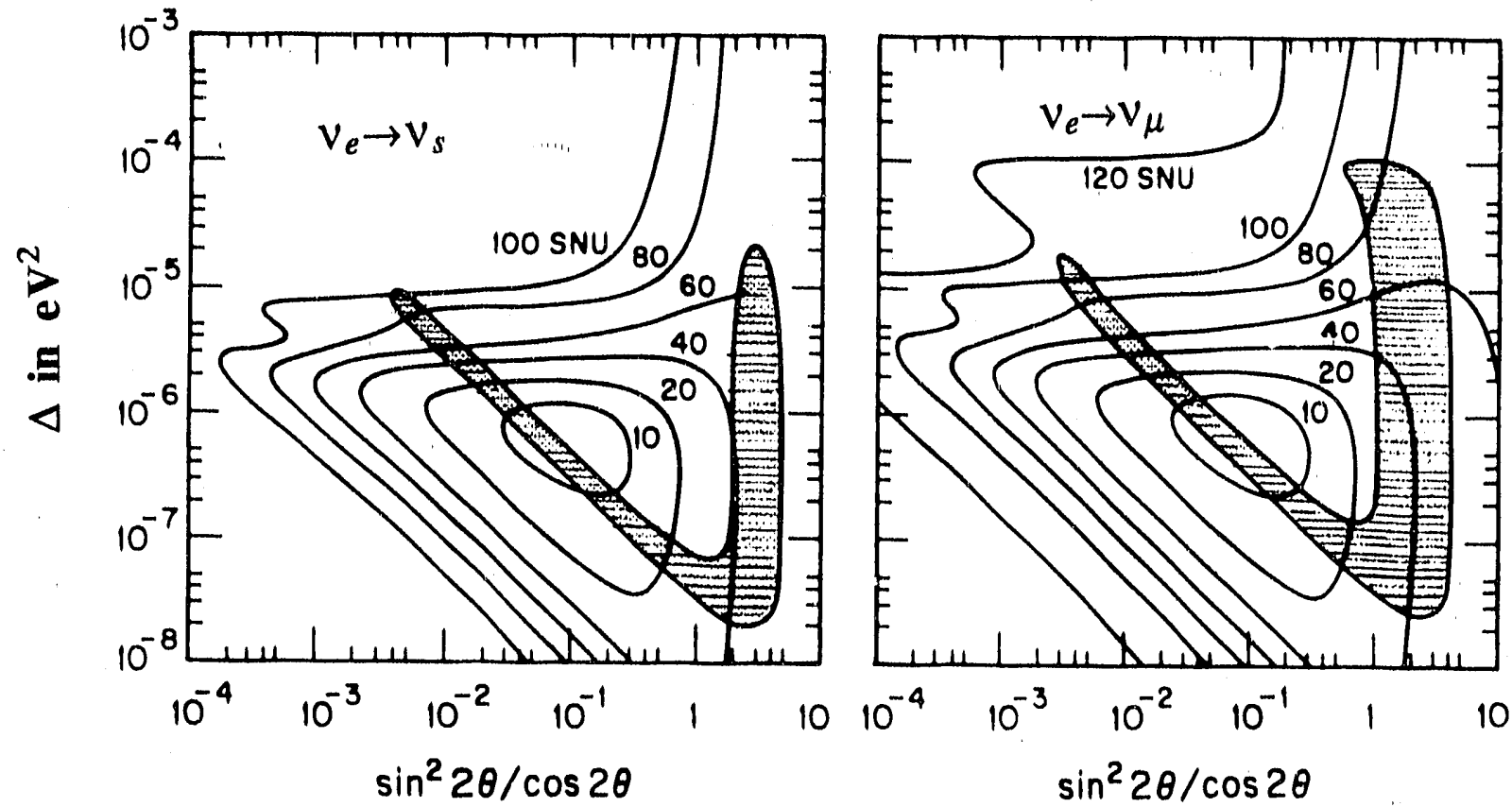

Figure 13: The allowed region of the parameter space if $\nu_{e}$ oscillates to a sterile neutrino. The shaded regions are allowed by both Chlorine and Kamiokande data. For ${ }^{71} \mathrm{Ga}$ detector, the standard solar model rate is taken to be $132 \mathrm{SNU}$.

If $\nu_{e}$ oscillates not to $\nu_{\mu}$ but to $\nu_{\tau}$, all the above analysis remain unchanged because $\nu_{\mu}$ and $\nu_{\tau}$ have same interactions in a medium which contains electrons but not muons or taons. However, in many extensions of the standard model including most grand unified models, there exist additional neutral fermion fields. In general, $\nu_{e}$ mixes with them and therefore can oscillate into them. What happens, for example, if $\nu_{e}$ oscillates to one such neutrino $\nu_{s}$ which is sterile, i.e., has no weak interactions?

If we want to find the solution by vacuum oscillations, the only difference is that, since the sterile neutrinos are not detected by the Kamiokande experiment, we should use $P_{\mathrm{Kam}}=P_{\nu_{e} \nu_{\mathrm{e}}}$ rather than Eq. (3.23). Thus, at the $95 \%$ confidence level, we should impose

$$
0.30<P_{\nu_{e} \nu_{e}}<0.62
$$

instead of the limits in Eq. (3.25). This results in a somewhat smaller allowed 
region [13].

In case of the matter enhanced oscillation as well, the change is formally simple. When we considered $\nu_{e}$ oscillating to $\nu_{\mu}$, we needed the effective hamiltonian for the $\nu_{e}-\nu_{\mu}$ system, which was given in Eq. (4.14). Recall that in that equation, $H^{\prime}$ is the hamiltonian in the vacuum, which has the same form if we considered the $\nu_{e}-\nu_{s}$ system as well. Among the terms coming from matter contributions, the term proportional to $n_{e}$ comes from charged current interactions, and will be unchanged in the $\nu_{e}-\nu_{s}$ case as well. However, in Eq. (4.14), the neutral current contriubitons affect both $\nu_{e}$ and $\nu_{\mu}$ equally. If we consider the $\nu_{e}-\nu_{s}$ system, $\nu_{s}$ will not have this term since, being sterile, it does not have weak interactions. Of course, $\nu_{e}$ will have the neutral current term as before. Thus, we have

$$
\widetilde{H}=H^{\prime}+\left(\begin{array}{cc}
\sqrt{2} G_{F} n^{\prime} & 0 \\
0 & 0
\end{array}\right) .
$$

where

$$
n^{\prime}=n_{e}-\frac{1}{2} n_{n}
$$

The analysis is exactly similar, except $n_{e}$ has to be replaced by $n^{\prime}$ everywhere. The solution space changes [23], as shown in Fig. 13. To show the difference clearly, we have presented the calculations for a ${ }^{71} \mathrm{Ga}$ detector as well.

\subsection{Solution with more than two generations of neutrinos}

It is straightforward to generalize the formulas obtained for the two generation case to include the effects of extra generations. For the vacuum oscillation case, we can write any flavor state in general as

$$
\left|\nu_{\ell}\right\rangle=\sum_{\alpha} U_{\ell \alpha}\left|\nu_{\alpha}\right\rangle
$$

where the index $\alpha$ runs over all the mass eigenstates. If one creates a beam of $\nu_{\ell}$ at $t=0$, its time evolution will be given by

$$
\left|\nu_{\ell}(t)\right\rangle=\sum_{\alpha} e^{-i E_{\alpha} t} U_{\ell \alpha}\left|\nu_{\alpha}\right\rangle,
$$

The probahility of finding the flavor state $\nu_{\ell^{\prime}}$ in this state is given by

$$
\begin{aligned}
P_{\nu_{\ell} \nu_{\ell^{\prime}}}(t) & =\left|\left\langle\nu_{\ell^{\prime}} \mid \nu_{\ell}(t)\right\rangle\right|^{2} \\
& =\sum_{\alpha, \beta}\left|U_{\ell \alpha} U_{\ell^{\prime} \alpha}^{*} U_{\ell \beta}^{*} U_{\ell^{\prime} \beta}\right| \cos \left[\left(E_{\alpha}-E_{\beta}\right) t-\varphi_{\ell \ell^{\prime} \alpha \beta}\right]
\end{aligned}
$$


where

$$
\varphi_{\ell \ell^{\prime} \alpha \beta}=\arg \left(U_{\ell \alpha} U_{\ell^{\prime} \alpha}^{*} U_{\ell \beta}^{*} U_{\ell^{\prime} \beta}\right)
$$

Using the approximation $E_{\alpha} \gg m_{\alpha}$ as before, we can use Eq. (3.6) to write $[11,44]$

$$
P_{\nu_{\ell} \ell_{\ell^{\prime}}}(x)=\sum_{\alpha, \beta}\left|U_{\ell \alpha} U_{\ell^{\prime} \alpha}^{*} U_{\ell \beta}^{*} U_{\ell^{\prime} \beta}\right| \cos \left(\frac{\Delta_{\alpha \beta}}{2 E} x-\varphi_{\ell \ell^{\prime} \alpha \beta}\right),
$$

where

$$
\Delta_{\alpha \beta} \equiv m_{\alpha}^{2}-m_{\beta}^{2}
$$

As in the case with two generations, there are some allowed regions where the $\Delta$-values are around $10^{-10} \mathrm{eV}^{2}$. But, with three generations, some new type of solutions are also possible.

For this, consider that we are in a range of values of $\Delta_{\alpha \beta}$ where $\left(\Delta_{\alpha \beta} / 2 E\right) x \geqslant 1$ for typical energies of solar neutrinos. The energy averaging then completely washes out the terms with $\alpha \neq \beta$ and we get

$$
\bar{P}_{\nu_{\ell} \ell_{\ell^{\prime}}}=\sum_{\alpha}\left|U_{\ell \alpha}\right|^{2}\left|U_{\ell^{\prime} \alpha}\right|^{2}
$$

To proceed, let us parametrize the elements of the mixing matrix by

$$
U_{e 1}=\cos \theta_{1}, \quad U_{e 2}=\sin \theta_{1} \cos \theta_{2}, \quad U_{e 3}=\sin \theta_{1} \sin \theta_{2} .
$$

Then,

$$
\bar{P}_{\nu_{\mathrm{e}} \mathrm{lic}_{\mathrm{e}}}=\cos ^{4} \theta_{1}+\sin ^{4} \theta_{1}\left(\cos ^{4} \theta_{2}+\sin ^{4} \theta_{2}\right)
$$

Unlike the 2-generation case where $\bar{P}_{\nu_{\mathrm{e}} \nu_{e}} \geq \frac{1}{2}$, here we can get $\bar{P}_{\nu_{\mathrm{e}} \nu_{\mathrm{e}}}$ as low as $\frac{1}{3}$. This can be consistent with the $2 \sigma$ survival probabilites found in Chlorine and Kamiokande experiments, as given in Eq. (3.22) and Eq. (3.25). The solution for the angles is shown in Fig. 14. The values of the mass square differences have to be much larger than $10^{-10} \mathrm{eV}^{2}$ and are bounded from above by terrestrial limits given in Appendix A.

It is easy to calculate the corresponding probabilities if we take matter effects into account as long as the propagation is adiabatic. Following the arguments leading to Eq. (5.11), we get

$$
P_{\nu \ell \ell^{\prime}}^{(\mathrm{ad})}=\sum_{\alpha}\left|\tilde{U}_{\ell \alpha}\right|^{2}\left|U_{\ell^{\prime} \alpha}\right|^{2}
$$

where $\tilde{U}$ is the effective mixing matrix at the point where the neutrino is produced. This looks deceptively simple. The cumbersome part involves the determination of 


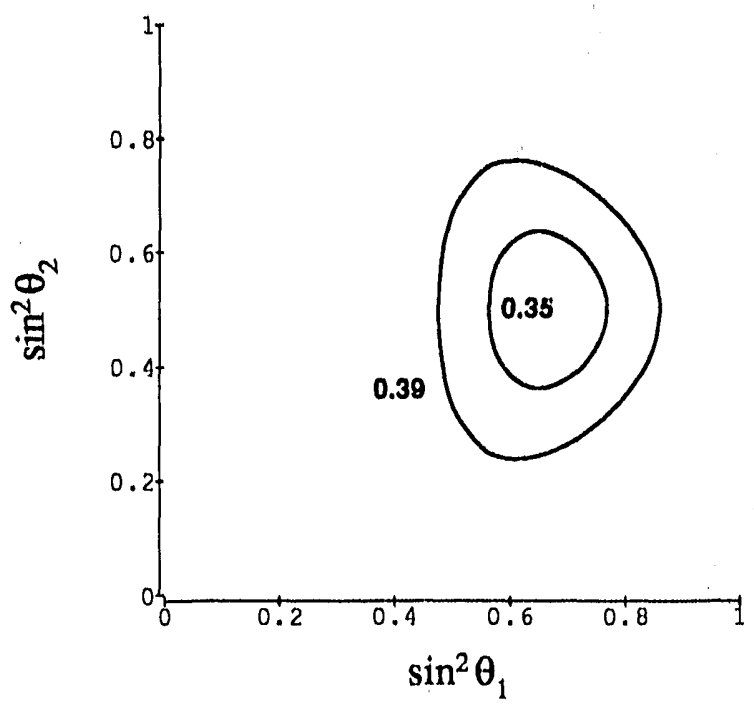

Figure 14: Vacuum oscillation solutions for the solar neutrino problem with three generations of neutrinos. The mass square differences are assumed to be $\gg 10^{-10} \mathrm{eV}^{2}$. The inside of the inner and outer curves are consistent with the data at the $2 \sigma$ and $3 \sigma$ levels respectively.

the elements of $\tilde{U}$ in terms of the density of the medium and energy of the neutrinos. The exact analysis for the three generation case $[45,46]$ is quite complicated since it involves the solution of a cubic equation. Some approximate solution techniques have also been tried $[47,48,49,50,51,52,53,54,55,56]$, which give more intuitive feeling for the solution.

From the discussion of the two generation case, it is obvious that two resonances will occur in the three generation case. In a diagram like Fig. 6, these resonances take place when $\left\langle E_{\nu_{e}}\right\rangle$ crosses $\left\langle E_{\nu_{\mu}}\right\rangle$ and $\left\langle E_{\nu_{\tau}}\right\rangle$ respectively. When the two crossings are fairly far apart, the two resonances can be treated independently of each other. But even then, confrontation of the data is quite involved for many reasons. The solution space is much bigger since it involves two mass squared differences $\Delta_{21}$ and $\Delta_{31}$, as well as three mixing angles. In general, even CP-violating phases can appear in the mixing matrix, making the problem more frustrating. Moreover, the results of two experiments might not be correlated in the sense that one of them might show a depletion in flux because of $\nu_{e}$ 's converting to $\nu_{\mu}$ 's, whereas the other one might show a depletion because of $\nu_{e}$ 's converting to $\nu_{\tau}$ 's.

Non-adiabatic effects will make the problem further complicated. They introduce transitions between different stationary states. If we denote the transition 
probability from a state $\tilde{\nu}_{\alpha}$ to a state $\tilde{\nu}_{\beta}$ by $X_{\alpha \beta}$, then

$$
P_{\nu_{\ell} \ell^{\prime}}=\sum_{\alpha, \beta}\left|\tilde{U}_{\ell \alpha}\right|^{2}\left|U_{\ell^{\prime} \beta}\right|^{2} X_{\alpha \beta}
$$

For $N$ generations, there are at most $(N-1)^{2}$ independent quantities $X_{\alpha \beta}$ since they must satisfy the relations $\sum_{\alpha} X_{\alpha \beta}=1$ and $\sum_{\beta} X_{\alpha \beta}=1$ which follow from unitarity. However, the quantities $X_{\alpha \beta}$ cannot be calculated analytically in general. Some conjectures have beer made in the case where the resonances are well separated [57].

\section{Part III}

\section{Neutrino magnetic moment}

It has been argued from time to time $e^{\sharp 6}$ that the neutrino flux detected in the Chlorine experiment has shown some anticorrelation with sunspot activity. This is shown in Fig. 15, where we have plotted the Chlorine data alongwith the number of sunspots. Notice that the scale of sunspot numbers is reversed, so a correlation on this figure implies an anticorrelation in the physical quantities.

If this anticorrelation is believed to be real, perhaps the most reasonable explanation of the solar neutrino problem would involve a magnetic moment of the electron neutrino $[59,60,61]$. Qualitatively, the idea is as follows. Sunspot activity is related to magnetic field in the sun. If the magnetic field is large, sunspot activity is high. On the other hand, if neutrinos have a magnetic moment, they will undergo spin precession in a magnetic field. The neutrinos produced in the nuclear reactions are left handed. As they travel through a transverse magnetic field, they can precess to a right handed neutrino which has hardly any interaction and therefore cannot be detected. At the time of high sunspot activity, the magnetic field is larger, and so the probability of precession to a right handed neutrino is also larger. In other words, the probability of detecting a solar neutrino is smaller at the time of high sunspot activity since the left handed component in the neutrino beam is smaller. This results in an anticorrelation of neutrino flux and sunspot number.

In this part of this article, we will discuss the theoretical ramifications of the aforesaid anticorrelation, assuming that it really exists. As for this assumption we need to point out that although Fig. 15 indicates some anticorrelation, it is not clear whether it is statistically significant. Moreover, one needs to remember that

\footnotetext{
${ }^{16}$ For a recent analysis and older references, see e.g. Ref. [58].
} 


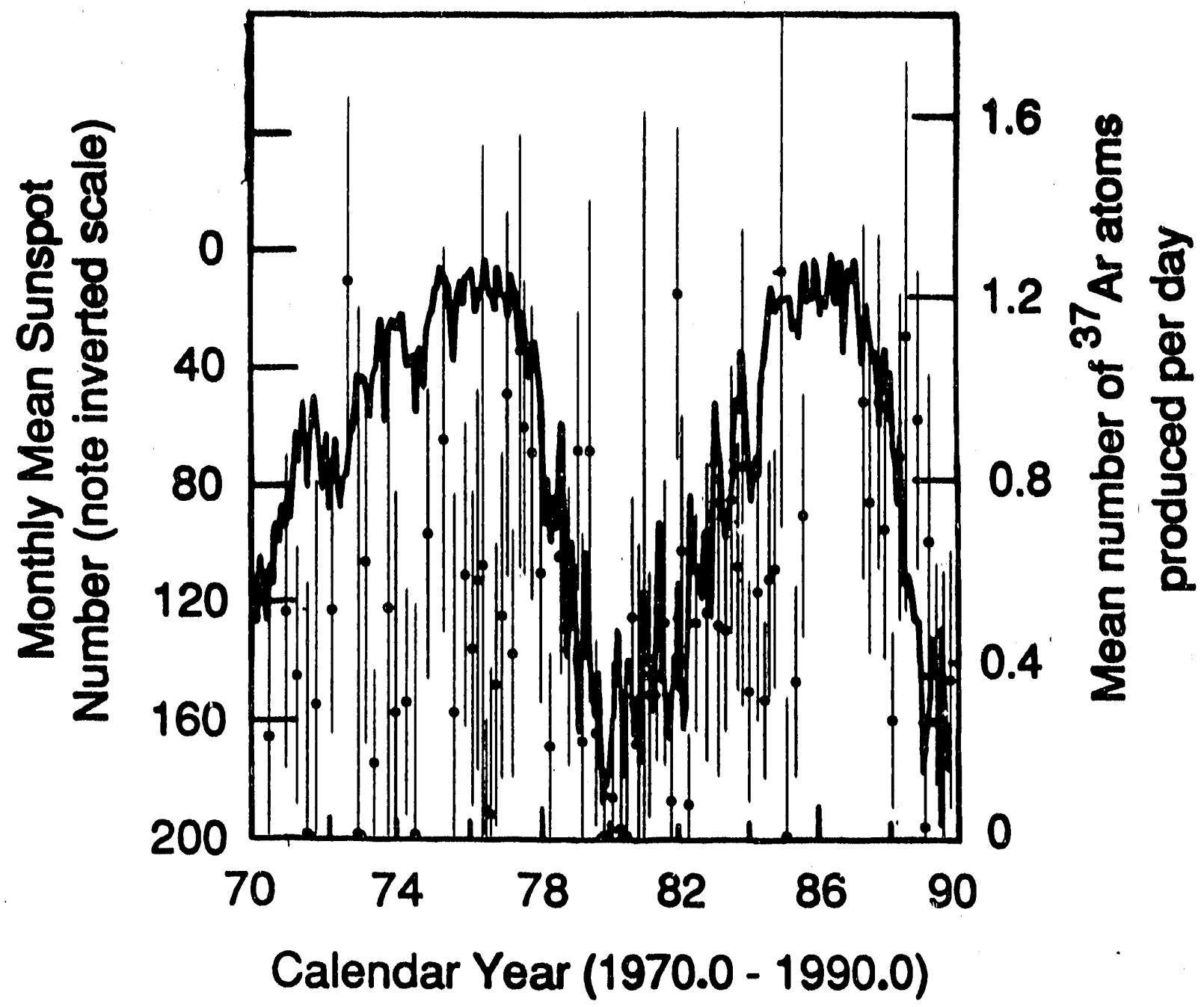

Figure 15: The continuous line denotes the number of sunspots, and the circles are the mean values of the ${ }^{37} \mathrm{Ar}$ produced in the Chlorine detector. The graph indicates some anticorrelation. Note the inverted scale for sunspot numbers. 
the figure shows only data from the Davis experiment. The Kamiokande data is fairly flat. Between 1988 and 1990 when the solar neutrino flux detected in the Chlorine experiment dropped noarly by a factor of 4 , the Kamiokande result varied by $10 \%$ at most. In some sense, this difference in behaviour is also a puzzle of the solar neutrinos, and some explanations have been suggested, as we will discuss in Sec. 12. For the most part, we will be concerned with the depletion of flux at the time of high sunspot activity.

\section{Kinematics of neutrino propagation in mag- netic field}

\subsection{Spin precession}

In quantum field theory, magnetic moment interaction between two fermion fields $\psi$ and $\psi^{\prime}$ is given by $\mu \bar{\psi} \sigma_{\lambda \rho} \psi^{\prime} F^{\lambda \rho}$, where $F^{\lambda \rho}$ is the electromagnetic field tensor. If $\psi$ and $\psi^{\prime}$ are same fields, the interaction reduces to $\mu\langle\mathbf{s}\rangle \cdot \mathbf{B}$ in the non-relativistic approximation, where $\mathbf{B}$ is the magnetic field and $\langle\mathbf{s}\rangle$ is the expectation value of spin. This identifies $\mu$ as the magnetic moment of the particle. When $\psi$ and $\psi^{\prime}$ represent different fields, the coefficient $\mu$ in that case is called a transition magnetic moment.

In a background field $\left\langle F^{\lambda \rho}\right\rangle$, the effect of magnetic moment $\mu$ is to add a term $\mu \bar{\psi} \sigma_{\lambda \rho} \psi^{\prime}\left\langle F^{\lambda \rho}\right\rangle$ to the free Lagrangian of a particle. This term connects opposite chiralities since

$$
\bar{\psi} \sigma_{\lambda \rho} \psi^{\prime}=\bar{\psi}_{L} \sigma_{\lambda \rho} \psi_{R}^{\prime}+\bar{\psi}_{R} \sigma_{\lambda \rho} \psi_{L}^{\prime}
$$

Thus, these terms are similar to the mass terms in the sense that they contain no interaction or derivatives and they connect opposite chiralities.

Consider $[60,61]$ the system of a left handed neutrino $\nu_{L}$ and a right handed one, $\nu_{R}$. To keep the discussion general, we do not even assume that their masses are equal - we denote the masses by $m_{L}$ and $m_{R}$ respectively. In a magnntic field $B$, the evolution equation of this system is governed by the equation:

$$
\begin{aligned}
i \frac{d}{d x}\left(\begin{array}{c}
\nu_{L} \\
\nu_{R}
\end{array}\right) & =\left[|\mathbf{p}|+\left(\begin{array}{cc}
m_{L}^{2} / 2|\mathbf{p}| & \mu B \\
\mu B & m_{R}^{2} / 2|\mathbf{p}|
\end{array}\right)\right]\left(\begin{array}{l}
\nu_{L} \\
\nu_{R}
\end{array}\right) \\
& =\left[\left(\begin{array}{cc}
\Delta_{L R} / 4 E & \mu B \\
\mu B & -\Delta_{L R} / 4 E
\end{array}\right)+\text { (unit matrix terms) }\right]\left(\begin{array}{l}
\nu_{L} \\
\nu_{R}
\end{array}\right)
\end{aligned}
$$

where

$$
\Delta_{L R}=m_{L}^{2}-m_{R}^{2}
$$


and as usual we have denoted $|\mathbf{p}|$ by $E$.

As in the case of the oscllation formalism, we can omit terms proportional to the unit matrix in the hamilltoulan without affecting any probabilities. Thus, we write

$$
i \frac{d}{d x}\left(\begin{array}{l}
\nu_{L} \\
\nu_{R}
\end{array}\right)=\left(\frac{\Delta_{L R}}{4 E} \sigma_{3}+\mu B \sigma_{1}\right)\left(\begin{array}{l}
\nu_{L} \\
\nu_{R}
\end{array}\right)
$$

For a uniform magnetic field, this equation can be formally integrated and one obtains the solution:

$$
\begin{aligned}
\left(\begin{array}{l}
\nu_{L}(x) \\
\nu_{R}(x)
\end{array}\right) & =\exp \left[-i\left(\frac{\Delta_{L R}}{4 E} \sigma_{3}+\mu B \sigma_{1}\right) x\right] \cdot\left(\begin{array}{l}
\nu_{L}(0) \\
\nu_{R}(0)
\end{array}\right) \\
& =\left[\cos \Omega x-\frac{i}{\Omega}\left(\frac{\Delta_{L R}}{4 E} \sigma_{3}+\mu B \sigma_{1}\right) \sin \Omega x\right]\left(\begin{array}{l}
\nu_{L}(0) \\
\nu_{R}(0)
\end{array}\right)
\end{aligned}
$$

where

$$
\Omega^{2} \equiv(\mu B)^{2}+\left(\Delta_{L R} / 4 E\right)^{2} .
$$

If we produce a beam of $\nu_{L}$ at $x=0$ and let it travel through the magnetic field, the spin direction of the beam would undergo a precession. The probability of finding a $\nu_{L}$ in this beam at the point $x$ is given by

$$
\begin{aligned}
P_{\nu_{L} \nu_{L}}(x) & =\left|\left\langle\nu_{L}(x) \mid \nu_{L}(0)\right\rangle\right|^{2} \\
& =\left|\cos \Omega x-\frac{i}{\Omega} \frac{\Delta_{L R}}{4 E} \sin \Omega x\right|^{2} \\
& =\cos ^{2} \Omega x+\cos ^{2} \beta \sin ^{2} \Omega x .
\end{aligned}
$$

In the last step, we have introduced the angle $\beta$, which is defined by

$$
\tan \beta \equiv \frac{\mu B}{\Delta_{L R} / 4 E}
$$

Spin precession is efflcient when $\beta \gtrsim 1$, i.e., when

$$
\Delta_{L R} \lesssim 4 E \mu B \text {. }
$$

Let us try to estimate how large the right hand side can be. The magnetic moment is an effective neutrino-photon coupling through which the neutrinos can interact with electrons. Laboratory measurements on neutrino-electron scattering puts the bound $|\mu| \lesssim 10^{-10} \mu_{B}$. For solar neutrinos, the energy is of order $10 \mathrm{MeV}$ or less. The magnetic field in the sun is not well-known at all. Educated guesses give $B \sim 10^{3}$ to $10^{4}$ Gauss. Using these values, wo obtain [61]

$$
\left|\Delta_{L R}\right| \lesssim 10^{-7} \mathrm{eV}^{2} .
$$


For a siugle Dirac neutrino, this is trivially satisfied in the vacuum since $\Delta_{L R}=0$. The problem then reduces to the canonical problem of spin precession treated in any elementary textbook of quantum mochanics. The present situation is a little bit different since the magnetic field exists not in the vacuum but in the solar material. So, the masses relevant sor this problem are not the masses in the vacuum, but rather the effective masses inside the medium. As described in Sec. 4, the effective masses receive contributions due to interactions in the medium. Borrowing the results proved in that context, we write

$$
\begin{aligned}
& m_{L}^{2}=m^{2}+2 \sqrt{2} G_{F^{\prime}} E\left(n_{e}-\frac{1}{2} n_{n}\right) \\
& m_{R}^{2}=m^{2}
\end{aligned}
$$

where $m$ is the vacuum mass. Ncte that for $\nu_{R}$, this is the same as the effective mass since $\nu_{R}$ does not have weak interactions. Thus, $\Delta_{L R}=2 \sqrt{2} G_{F} E\left(n_{e}-\frac{1}{2} n_{n}\right)$, and putting it in Eq. (8.9), we obtain for energies in the 1 to $10 \mathrm{MeV}$ range,

$$
n_{e}-n_{n} \lesssim 10^{22} \mathrm{~cm}^{-3}
$$

This is possible in the outer part of the sun (convective zone).

\subsection{Spin-flavor oscillations and resonance ${ }^{\dagger}$}

Relaxation of the upper bound on $\Delta_{L R}$ (or consequently, on densities) can be obtained if more than one flavor is considered. Consider two Dirac neutrinos. In the flavor states, we now will have to include the right handed fields also to see the effect of the magnetic field. Thus,

$$
\boldsymbol{\nu}^{(\mathrm{f})}=\left(\begin{array}{c}
\nu_{e L} \\
\nu_{\mu L} \\
\nu_{e R} \\
\nu_{\mu R}
\end{array}\right)
$$

The evolution equation is given by

$$
i \frac{d}{d x} \nu^{(f)}=\mathcal{H} \nu^{(f)}
$$

where $[62,63]$

$$
\mathcal{H}=\left(\begin{array}{cccc}
-\frac{\Delta}{4 E} \cos 2 \theta+V_{\nu_{e}} & \frac{\Delta}{4 E} \sin 2 \theta & \mu_{e e} B & \mu_{e \mu} B \\
\frac{\Delta}{4 E} \sin 2 \theta & \frac{\Delta}{4 E} \cos 2 \theta+V_{\nu_{\mu}} & \mu_{\mu e} B & \mu_{\mu \mu} B \\
\mu_{e e}^{*} B & \mu_{\mu e}^{*} B & -\frac{\Delta}{4 E} & 0 \\
\mu_{e \mu}^{*} B & \mu_{\mu \mu}^{*} B & 0 & \frac{\Delta}{4 E}
\end{array}\right)
$$




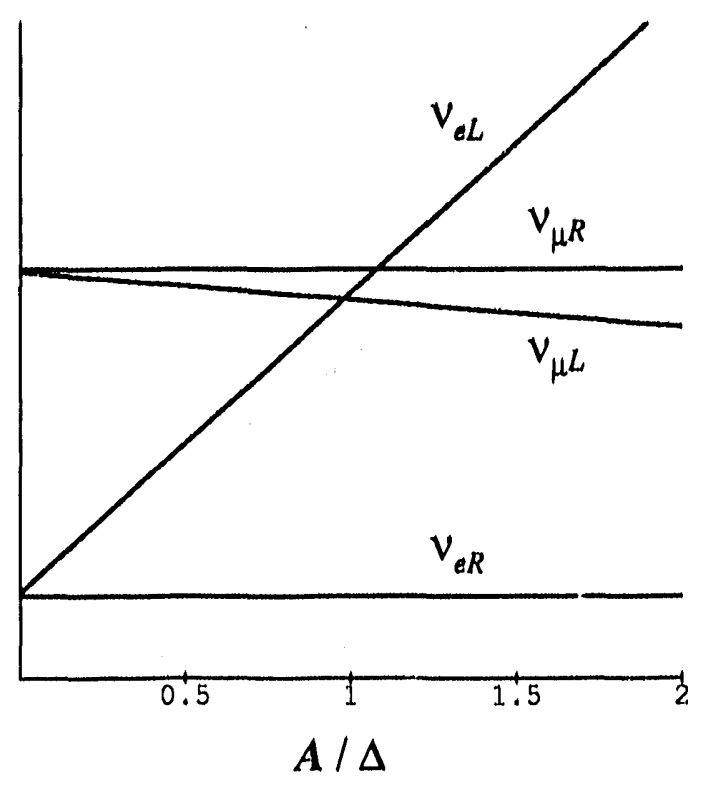

Figure 16: The expectation values of the mass squared operator for different flavor states. The crossings on this plot denote resonances. The vertical scale is arbitrary.

We have, of course, taken the liberty of omitting a term proportional to the unit matrix. The quantity $\Delta$ here is the mass square difference of the two physical Dirac neutrinos in the vacuum, and $\theta$ is the vacuum mixing angle. The various magnetic moments have been represented by the quantities $\mu_{\ell \ell^{\prime}}$ where $\ell, \ell^{\prime}=\mu$ or $e$. Finally, $V_{\nu_{e}}$ and $V_{\nu_{\mu}}$ are the potentials felt by left handed $\nu_{e}$ and $\nu_{\mu}$, which were given in Eq. (4.9). Thus the upper left $2 \times 2$ block of the matrix $\mathcal{H}$ is the same as the Hamiltonian $\widetilde{H}$ discussed in the context of neutrino oscillation in matter. The lower $2 \times 2$ block has no matter contribution since the right handed neutrinos have no interaction with matter. If the neutrinos are Majorana particles rather than Dirac ones, the right handed objects would really be the right handed antineutrinos, so that even the lower block will have matter corrections.

In Fig. 16, we have shown the plot of diagonal elements of $\mathcal{H}$ assuming $n_{n} \simeq \frac{1}{6} n_{e}$, which is approximately valid everywhere in the sun except the inner $20 \%$ of the radius. From the plot, we see two crossings of diagonal elements, which are the regions where resonances occur. Thus, the $\nu_{e L}-\nu_{\mu R}$ resonance occurs when

$$
V_{\nu_{\mathrm{e}}}=\frac{\Delta}{4 E}(1+\cos 2 \theta)
$$

which gives the condition

$$
\left(n_{e}-\frac{1}{2} n_{n}\right)_{R}=\frac{\Delta \cos ^{2} \theta}{2 \sqrt{2} G_{F} E}
$$


On the other hand, the $\nu_{e} L-\nu_{\mu L}$ resonance (or the usual MSW) resonance, occurs when

$$
\left(n_{e}\right)_{R}=\frac{\Delta \cos 2 \theta}{2 \sqrt{2} G_{F} E} .
$$

For $\theta \ll 1$ the two resonances are close since in the sun, $n_{n} \ll n_{e}$. Because of this, one cannot use the analytic solutions obtained in Part II for a single resonance. To proceed, one can make some simplifying assumptions. For example, if the mixing angle $\theta$ is zero, and if the magnetic moments are purely transitional (i.e., $\mu_{e e}=$ $\mu_{\mu \mu}=0$ ), the $4 \times 4$ matrix of Eq. (8.15) decomposes into two $2 \times 2$ blocks. The block involving $\nu_{e L}$ can then be analyzed $[64,65]$ in much the same way as the MSW solution was analyzed. Of course, to do this, one has to make some plausible guesses about the nature of variation of the magnetic field inside the sun. The experimental limits on survival probability will select out regions in the parameter space of $\Delta$ vs the transition magnetic moment. From such analysis, one finds that it is possible to have solutions for $\Delta$ as high as $10^{-4} \mathrm{eV}^{2}$.

In the general case when the mixing does not vanish, the only recourse is numerical solutions. For various acceptable values of the vacuum mixing angle $\theta$, the magnetic field $B$ and the magnetic moments, numerical solutions were performed $[62,63]$. They also show that values of $\Delta_{L R}$ as high as $10^{-4} \mathrm{eV}^{2}$ can be acceptable. But for large $\Delta$, the resonance occurs in the radiation zone (further inside). This may not be related to sunspot activities. So let us, for the moment, stick to the single flavor case discussed earlier.

\subsection{How large a magnetic moment do we need?}

For the single flavor case, we recall the solution of the evolution equation in Eq. (8.5). Using it, we found in Eq. (8.7) the probability of finding a $\nu_{L}$ after a $\nu_{L}$ beam travels through a distance $x$. Equivalently, we can write down the expression for the probability of finding a $\nu_{R}$ in that beam:

$$
P_{\nu_{L} \nu_{R}}(x)=1-P_{\nu_{L} \nu_{L}}(x)=\sin ^{2} \beta \sin ^{2} \Omega x .
$$

To obtain substantial flip, one not only needs $\beta \gtrsim 1$ but also $\Omega x \sim 1$. So far, we have discussed the consequences of the condition $\beta \gtrsim 1$ and found that $\Delta_{L R}$ has to be small. Because of that, $\Omega \sim \mu B$, so that the second condition reads $\mu B x \sim 1$. Using the eliucated guess that $B \sim 10^{3}$ to $10^{4}$ Gauss in the convective zone whose width is $x \sim 2 \times 10^{10} \mathrm{~cm}$, we obtain

$$
\mu \simeq(0.1 \text { to } 1.0) \times 10^{-10} \mu_{B} .
$$


Previously, we mentioned the neutrino-electron scattering puts upper bounds of order $10^{-10} \mu_{B}$ on neutrino magnetic moment. Here, we see that the magnetic moment cannot be far from that upper bound if it has to provide the solution of the solar neutrino puzzle. The question to ask then is: could neutrinos have magnetic moment in this range for viable models of particle interactions?

Before entering into a discussion of this question in the rest of this part of the article, we want to emphasize that the estimate of Eq. (8.20) has been based on a specific estimate of the magnetic field. The magnetic fileds inside the sun are not very well known. It has been argued that at the time of sunspot activities, the field becomes as strong as $10^{6}$ Gauss at some places [66]. If such a strong field exists over a large region, we can obtain substantial spin flip with a magnetic moment much smaller that what is specified in Eq. (8.20). It has also been argued [67] that apart from the dynamical factor appearing in the neutrino propagation, which was shown in Eq. (8.5), there can also be topological Berry phases, which can make the spin precession more efficient so that magnetic moments as small as $\sim 10^{-13} \mu_{B}$ may be sufficent to solve the solar neutrino problem.

Thus, the magnitude of the magnetic moment needed to solve the problem can be debated upon. But in any case, the value that emerges is orders of magnitude larger than what one would expect from the most obvious extension of the standard model, which we discuss in Sec. 9.1. It is in this spirit that we approach the problem.

\section{Easy models for neutrino magnetic moment}

\subsection{The simplest attempt}

In the standard model, all neutrinos are massless. There is no right handed neutrino field. Because of this, neutrinos cannot have any magnetic moment as well. Thus, a neutrino magnetic moment calls for going beyond the standard model.

The simplest way to accommodate a neutrino magnetic moment is to add, to each generation of fermions of the standard moeld, a right handed neutrino field $\nu_{R}$. Such a state would be a singlet of the gauge group $S U(2)_{L} \times U(1)_{Y}$, and therefore will not have any gauge interaction. However, they will have Yukawa couplings of the form

$$
-\mathcal{L}_{Y}^{\prime}=h \bar{\psi}_{L} \varphi \nu_{R}+h . c .
$$

where $\psi_{L}$ denotes the doublet of left-handed leptons, and $\varphi$ is the Higgs doublet. We have suppressed the generation indices in the above equation. 

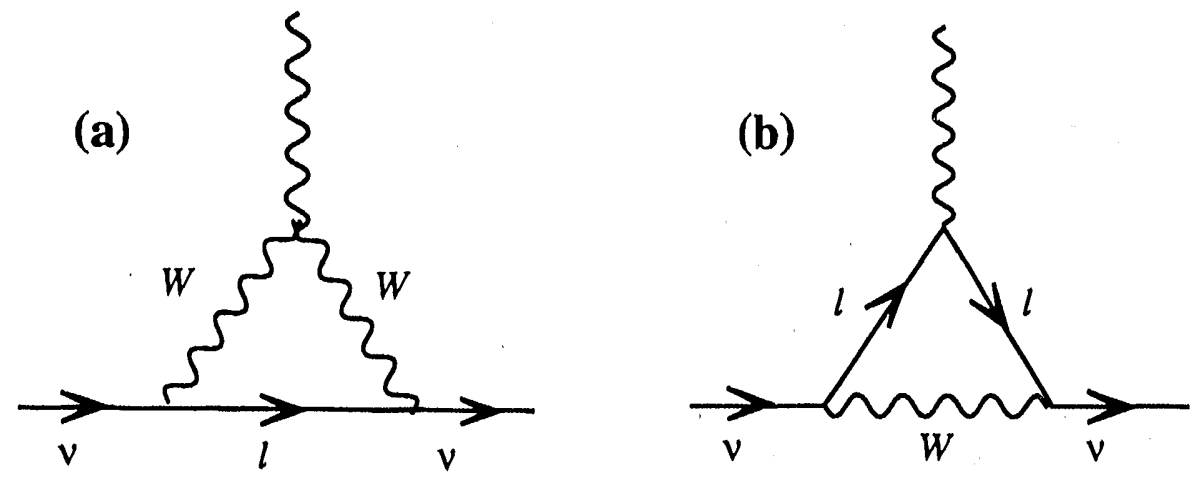

Figure 17: 1-loop diagrams mediated by $W$-bosons which give rise to magnetic moments of neutrinos.

The Yukawa coupling given above produces neutrino mass $m_{\nu}$ when $\varphi$ develops a vacuum expectation value. The magnetic moment arises at 1-loop through the diagrams shown in Fig. 17. It is easy to make a rough order-of-magnitude estimate of the magnetic moment generated by these diagrams. The loop integration typically comes with a factor of order $1 / 8 \pi^{2}$. The photon coupling would contribute a factor $e$. The couplings and propagators of $W$ give the Fermi constant $G_{F}$. And then, it is also clear that the magnetic moment will have a factor of $m_{\nu}$. This is because the $\nu_{R}$ does not have any interaction with the $W$. Thus, the only way to have the $\nu_{R}$ on the outer line of the diagram is by having $\nu_{R}$ changing the chirality through the mass to $\nu_{L}$, which participates in weak interactions. Taking all these factors together, we conclude

$$
\mu_{\nu} \sim \frac{1}{8 \pi^{2}} e G_{F} m_{\nu} .
$$

Detailed calculation supports this estimate and gives [68]

$$
\mu_{\nu}=\frac{3}{8 \sqrt{2} \pi^{2}} e G_{F} m_{\nu}=3 \times 10^{-19} \mu_{B} \cdot\left(\frac{m_{\nu}}{1 \mathrm{eV}}\right) .
$$

Since the $\nu_{e}$ mass cannot be larger than about $12 \mathrm{eV}$, we cannot get a magnetic moment much higher than about $10^{-18} \mu_{B}$ in this model. Compared to this benchmark value, we need a huge magnetic moment, as we menioned earlier.

\subsection{Naive Higgs models}

To show that it is not impossible to cook up models with large magnetic moments, we add to the particle content of the standard model not just the right handed neutrinos $\nu_{R}$ but also a charged scalar field $h_{+}$which is a singlet under the group $S U(2)_{L}$. This charged scalar field will have Yukawa couplings with the leptons: 


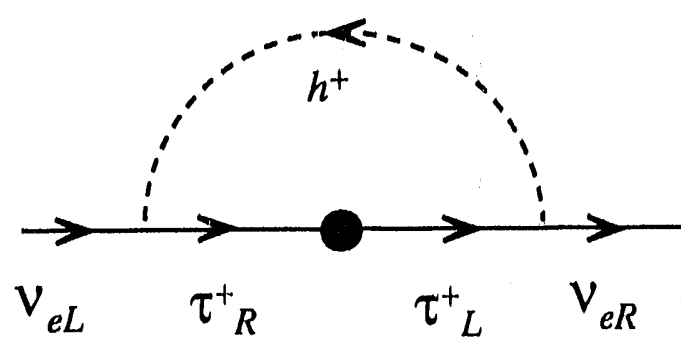

Figure 18: 1-loop diagrams involving Higgs boson exchange which gives the dominant contribution to neutrino magnetic moment in the model of Sec. 9.2. The photon line can be attached to any internal line. The blob denotes the $\tau$-masss.

$$
-\mathcal{L}_{Y}^{\prime}=\sum_{\ell, \ell^{\prime}}\left(f_{\ell \ell^{\prime}} \overline{\widehat{\psi}}_{\ell R} \psi_{\ell^{\prime} L}+f_{\ell \ell^{\prime}}^{\prime} \overline{\boldsymbol{\nu}}_{\ell L} \ell_{R}\right) h_{+}+\text {h.c. },
$$

where the hats denote conjugate fields. The diagram of Fig. 18 will now contribute to the magnetic moment of the neutrinos. Following the chirality of the fermion line, it is easy to see that the diagram is proportional to the mass of the internal fermion. Thus, it is expected that the largest contribution to the magnetic moment is obtained when the internal line corresponds to the $\tau$ lepion, and one gets $[69,70]$

$$
\mu_{\nu_{e}}=\frac{e f_{e \tau} f_{e \tau}^{\prime} m_{\tau}}{32 \pi^{2} M_{h}^{2}}\left(\ln \frac{M_{h}^{2}}{m_{\tau}^{2}}-1\right)
$$

The experimental constraints on the couplings $f_{a b}$ are very weak. One can have, e.g., $f_{e \tau} f_{e \tau}^{\prime} \sim 10^{-1}$ if $M_{h} \sim 100 \mathrm{GeV}$. In this case, the above equation gives $\mu_{\nu_{e}} \sim$ $10^{-10} \mu_{B}$.

While the model cannot be ruled out from experimental data, some weaknesses of the model are worth discussing. First, the model does not give any insight on the smallness of neutrino mases. In fact, neutrino masses have divergent contributions from diagrams like Fig. 18 without the photon line. These diveregences have to be renormalized by some suitable prescription which will determine the neutrino mass. Thus, from the point of view of the model, neutrino mass is completely arbitrary. Secondly, the right handed neutrinos, being gauge singlets, can have a bare Majorana mass term. If this term is present, the $\nu_{L}$ and the $\nu_{R}$, once the mass matrix is diagonalized, appear as chiral projections of two different Majorana particles. It is then hard to understand how to satisfy Eq. (8.9) without making some extreme assumptions about the bare mass of $\nu_{R}$. One has to therefore eliminate this term by postulating a global lepton number symmetry on the Lagrangian.

The model can be easily extended [71] to a left-right symmetric model based on the group $S U(2)_{L} \times S U(2)_{R} \times U(1)_{B-L}$, where $h_{+}$would be a singlet of $S U(2)_{L} \times$ 
$S U(2)_{R}$. In this case, the problem of bare masses is eliminated. However, one still needs to impose a global lepton number symmetry in order to obtain Dirac particles.

\section{Models with naturally large magnetic mo- ments}

\subsection{Generic problem with large magnetic moment}

The naive Higgs models described above can be tuned to give a small enough mass for a given magnetic moment, as we discussed in that context. However, such a procedure will suffer from unnatural fine tuning of parameters. To see this, let us consider a generic magnetic moment diagram. The contribution of the diagram is:

$$
\mu_{\nu} \sim \frac{e \mathcal{G}}{M}
$$

where $M$ denotes the heaviest mass in the loop, $e$ is of course the photon coupling, and $\mathcal{G}$ stands for everything else. The same diagram, stripped of the photon line, gives a contribution to neutrino mass. A naive order-of-magnitude estimate gives

$$
m_{\nu} \sim \mathcal{G} M .
$$

Combining these estimates, we get

$$
\frac{\mu_{\nu}}{m_{\nu}} \sim \frac{e}{M^{2}}
$$

Thus, if we want $\mu_{\nu_{e}} \sim 10^{-11} \mu_{B}$, we need

$$
M \lesssim 1 \mathrm{GeV} .
$$

since $m_{\nu_{e}}<12 \mathrm{eV}$. But, in the magnetic moment diagram, the internal line connecting to the photon must carry electric charge. Direct experimental searches show that the mass of any unknown charged particle must be larger than about $45 \mathrm{GeV}$. With $M \sim 45 \mathrm{GeV}$, if $\mu_{\nu_{e}} \sim 10^{-11} \mu_{B}$ then the natural value of $m_{\nu_{e}}$ turns out to be about $20 \mathrm{keV}$. One therefore has to perform some unnatural fine tuning to keep $m_{\nu_{e}}$ consistent with its experimental upper bound of $12 \mathrm{eV}$.

To get around this unnaturalness, one needs some symmetry in the theory. One can ask whether there is any symmetry in the models described above which can ensure $m_{\nu}=0$, but $\mu_{\nu} \neq 0$. There isn't any. The only symmetry broken by a neutrino mass term is a global chiral symmetry associated with the neutrino fields. But exactly the same symmetry is violated by the magnetic moment term. This 
shows up clearly in Eq. (10.1) and Eq. (10.2), where $\mathcal{G}$ is the chiral symmetry breaking parameter. Thus, once chiral symmetry is broken, one gets a nonzero $m_{\nu}$ as well. One needs some extra symmetry in the model in order to obtain $m_{\nu}=0$ for $\mu_{\nu} \neq 0$.

\subsection{Towards a better notation}

So far, we have written the magnetic moment operator as $\bar{\nu}_{R} \sigma_{\lambda \rho} \nu_{L}$ plus its hermitian conjugate. The mass operator, in the same customary notation, is $\bar{\nu}_{R} \nu_{L}+$ h.c.. We now want to write them in a different way which will be useful for later discussion.

Notice that the field operator $\bar{\nu}_{R}$ creates a right handed neutrino but also annihilates its antiparticle, the left handed antineutrino $\left(\widehat{\nu}_{L}\right)$. The same operations can be performed by the field operator of $\widehat{\nu}_{L}$ itself. In a matrix notation, one can write

$$
\bar{\nu}_{R}=\widehat{\nu}_{L}^{T} C,
$$

where $C$ is a numerical matrix that rearranges the rows and is unimportant as long as we consider the creation and annibilation properties only. Anyway, what Eq. (10.5) tells us that it is possible to write everything using left handed fields only. For example, the magnetic moment operator can be written as $\widehat{\nu}_{L}^{T} C \sigma_{\lambda \rho} \nu_{L}+$ h.c., the mass term as $\widehat{\nu}_{L}^{T} C \nu_{L}+$ h.c. This strategy of using fields with only one chirality is often used in grand unified model building. As we see now, here also the use of this notation gives some important insight.

\subsection{Suppressing $m_{\nu} / \mu_{\nu}$ by a symmetry}

Consider now the general case where there are a number of flavors of neutrinos. We gather all the uncharged left-handed fields, including things like $\widehat{\nu}_{L}$, and call them $\psi_{a L}$ where the index $a$ runs over a range of values. Writing all indices explicitly, the magnetic moment interaction can be written as

$$
\sum_{a, b} \sum_{\alpha, \beta} \mu_{a b}\left(\psi_{a L}\right)_{\alpha}\left(C \sigma_{\lambda \rho}\right)_{\alpha \beta}\left(\psi_{b L}\right)_{\beta} F^{\lambda \rho}
$$

where $\lambda, \rho$ are Lorentz indices and $\alpha, \beta$ denote various components of a spinor. In this notation, the most general mass term is given by

$$
\sum_{a, b} \sum_{\alpha, \beta} m_{a b}\left(\psi_{a L}\right)_{\alpha} C_{\alpha \beta}\left(\psi_{b L}\right)_{\beta} .
$$

The advantage of this notation is that, since we are dealing with bilinears involving the fields $\psi_{L}$ only (as opposed to bilinears invloving $\bar{\psi}$ as well), we can see 
the effect of Fermi statistics. If we interchange $\left(\psi_{a L}\right)_{\alpha}$ and $\left(\psi_{b L}\right)_{\beta}$, we expect a negative sign. In the mass term, interchanging the spinor indices $\alpha, \beta$ gives a sign since $C_{\alpha \beta}=-C_{\beta \alpha}$, i.e., $C$ is an antisymmetric matrix. In that term, then, exchange of the flavor indices $a, b$ must not give any extra sign, i.e., we need

$$
m_{a b}=m_{b a}
$$

to ensure Fermi statistics. On the other hand, we have the matrix $C \sigma_{\lambda \rho}$ in the magnetic moment term. Using the relation

$$
C \gamma_{\lambda} C^{-1}=-\gamma_{\lambda}^{T}
$$

which defines the matrix $C$, and t"ze equation

$$
\sigma_{\lambda \rho}=\frac{i}{2}\left[\gamma_{\lambda}, \gamma_{\rho}\right]
$$

which defines $\sigma_{\lambda \rho}$, it is easy to see that $C \sigma_{\lambda \rho}$ is symmetric in the spinor indices, i.e.,

$$
\left(C \sigma_{\lambda \rho}\right)_{\alpha \beta}=\left(C \sigma_{\lambda \rho}\right)_{\beta \alpha} .
$$

Thus, in Eq. (10.6), the negative sign due to the interchange of two spinors must come from their flavor indices, i.e., we need

$$
\mu_{a b}=-\mu_{b a}
$$

Voloshin [72] realized that these different symmetry properties of the mass and magnetic moment might be crucial to explain a large magnetic moment for a small mass. Consider the simple case where there are only two left-handed fields $\nu_{L}$ and $\widehat{\nu}_{L}$, and they form a doublet under some $S U(2)_{\nu}$ symmetry. Then the mass term, being the symmetric combination, would transform like a triplet where the antisymmetric magnetic moment would be a singlet. Thus, if this $S U(2)_{\nu}$ symmetry is an exact symmetry of the Lagrangian of a model, massless neutrinos can have nonzero magnetic moments in that model. As we will see next, the $S U(2)_{\nu}$ is broken in realistic models, which gives rise to nonzero masses as well. However, if the $S U(2)_{\nu}$ breaking is small in some sense, the ratio $m_{\nu} / \mu_{\nu}$ can be kept small, which is necessary for a natural explanation for a large magnetic moment of $O\left(10^{-11} \mu_{5}\right)$.

\subsection{Implementing the Voloshin symmetry}

Implementation of Voloshin's idea [72] is problematic since $S U(2)_{\nu}$ does not commute with the electroweak group. Several possibilities can be explored. 
Way 1: Enlarge electroweak group to include $S U(2)_{\nu}$. An example of such a model has been presented in Sec. 10.4.1.

Way 2: Use some other $S U(2)$ for Voloshin mechanism, which commutes with the gaigged $S U(2)_{L}$. An example is a horizontal symmetry $S U(2)_{H}$ with $\nu_{e L}, \nu_{\mu L}$ as a doublet $[73,74,75]$. The magnetic moment in this case connects $\nu_{e L}$ with $\nu_{\mu L}$, i.e., is transitional. Since $\nu_{e L}$ is part of the doublet $\psi_{e L}$ of the gauged $S U(2)_{L}$ and $\nu_{\mu L}$ is part of the doublet $\psi_{\mu L}$, we must put $\psi_{e L}$ and $\psi_{\mu L}$ as a horizontal doublet. Thus, in the limit of unbroken $S U(2)_{H}$, one obtains $m_{e}=m_{\mu}$. Such $S U(2)_{H}$ must therefore be broken to get $m_{e} \neq m_{\mu}$. The subgroup $L_{e}-I_{\mu}$ can remain unbroken.

Way 3: One can use a discrete symmetry with carefully chosen quantum numbers. To see how this works, consider [76] what could happen if the theory were invariant under charge conjugation $\mathcal{C}$, with

$$
\mathcal{C}\left(\nu_{e}\right)=-1, \quad \mathcal{C}\left(\nu_{\mu}\right)=+1 .
$$

The cross mass term $\nu_{e}^{T} C \nu_{\mu}$ would then be odd under $\mathcal{C}$, but the magnetic moment interaction $\nu_{e}^{T} C \sigma_{\lambda \rho} \nu_{\mu} F^{\lambda \rho}$ would have been even, i.e., invariant under $\mathcal{C}$ owing to a extra minus sign coming from the odd $\mathcal{C}$ properties of the electromagnetic field. Thus, the magnetic moment term would have been allowed by such a symmetry but the mass term wouldn't have. Of course, $\mathcal{C}$ is violated nearly maximally in weak interactions, so in realistic models, the above argument does not work. But, if we have some other discrete symmetry having similar property, it can suppress $m_{\nu}$.

Way 4: One can try to find a discrete nonabelian symmetry with same symmetry properties as $S U(2)_{\nu}$, i.e., under which the magnetic moment term is invariant but the mass term is not. There are various nonabelian discrete symmetries satisfying this condition $[77,78,79,80]$. We give some details of one such model in Sec. 10.4.2.

Below, we give some examples of models implementing the ideas above. The particular models chosen have no bearing on their physical plausibility. They are presented for illustrative purposes only.

\subsubsection{Example: Extended electroweak symmetry ${ }^{\dagger}$}

As an example of implementing Voloshin's mechanism with extended electroweak group, we consider a model described by Barbieri and Mohapatra [81] where electroweak interactions are described by the gauge group $S U(3)_{L} \times U(1)_{X}$. This group 

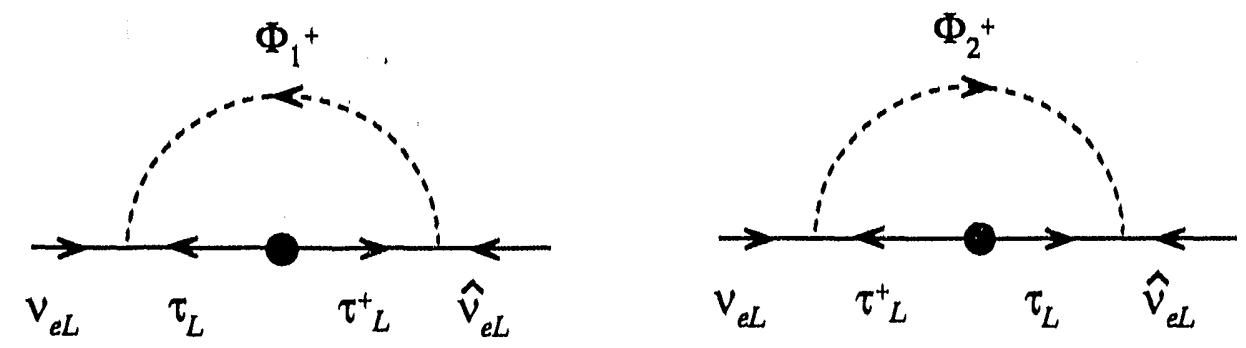

Figure 19: 1-loop diagrams involving Higgs boson exchange in the model of Ref. [81] which gives the dominant contribution to neutrino magnetic moment when a photon is attached to any of the internal lines.

spontaneously breaks down to $U(1)_{Q}$ of electromagnetism, with the charge operator given by $Q=T_{3}-\frac{1}{\sqrt{3}} T_{8}+X$, where

$$
T_{3}=\frac{1}{2} \operatorname{diag}(0,1,-1), \quad T_{8}=\frac{1}{2 \sqrt{3}} \operatorname{diag}(-2,1,1) .
$$

For a triplet, the electric charges are $\left(X+\frac{1}{3}, X+\frac{1}{3}, X-\frac{2}{3}\right)$. The leptons in a generation are assigned the following representations of the gauge group:

$$
\begin{gathered}
\Psi_{e L}=\left(\begin{array}{c}
\widehat{\nu}_{e L} \\
\nu_{e L} \\
e_{L}
\end{array}\right):\left(3,-\frac{1}{3}\right) \\
e_{L}^{+}:(1,1) .
\end{gathered}
$$

Looking at $\Psi_{e L}$, it is apparent that Voloshin's $S U(2)_{\nu}$ acts on the first two rows of the triplet, whereas the $S U(2)_{L}$ of the standard model acts on the last two rows. The representations of quarks are irrelevant for our purpose. The same can be said about some extra fermions which are necessary to ensure that the gauge anomalies cancel.

In the Higgs sector, first of all there is a triplet $\varphi$ :

$$
\varphi=\left(\begin{array}{c}
\varphi_{1}^{+} \\
\varphi_{2}^{+} \\
\varphi^{0}
\end{array}\right):\left(3, \frac{2}{3}\right)
$$

so that $\left\langle\varphi^{0}\right\rangle$ generates the mass of the electron and other charged fermions. There is another multiplet $\Phi$ which has the same gauge properties as $\phi$, but $\left\langle\Phi^{0}\right\rangle=0$. These two Higgs multiplets have various Yukawa couplings, among which the following terms are important for us:

$$
-\mathcal{L}_{Y}=h \Psi_{e L} \varphi^{*} e_{L}^{+}+f \Psi_{e L} \Psi_{\tau L} \Phi+f^{\prime} \Psi_{e L} \Phi^{*} \tau_{L}^{+} .
$$


In this equation, we have suppressed all $S U(3)_{L}$ indices. If we put them explicitly, the term whose coefficient is $f$ will look like

$$
\varepsilon_{\alpha \beta \gamma} \Psi_{e L}^{\alpha} \Psi_{\tau L}^{\beta} \Phi^{\gamma}
$$

There are two 1-loop diagrams for neutrino mass, as shown in Fig. 19. Each has one vertex involving this interaction. One of these has $\alpha=2, \beta=3, \gamma=1$, while the other has $\alpha=1, \beta=3, \gamma=2$. The antisymmetric $\varepsilon$-symbol then gives a relative negative sign between the contributions of the two diagrams, so that we get

$$
m_{\nu_{\mathrm{e}}} \simeq \frac{f f^{\prime}}{16 \pi^{2}} m_{\tau} \ln \frac{m_{\Phi_{1}}^{2}}{m_{\Phi_{2}}^{2}}
$$

The diagrams for magnetic moment are obtained by attaching a photon to ary internal line. The photon attaches to particles of oppostie charges in the two diagrams, which cancels the negative sign from the $\varepsilon$-symbol. Thus,

$$
\mu_{\nu_{e}} \simeq \frac{e f f^{\prime}}{16 \pi^{2}} m_{\tau}\left[\frac{1}{m_{\Phi_{1}}^{2}} \ln \frac{m_{\Phi_{1}}^{2}}{m_{\tau}^{2}}+\frac{1}{m_{\Phi_{2}}^{2}} \ln \frac{m_{\Phi_{2}}^{2}}{m_{\tau}}\right] .
$$

If $S U(2)_{\nu}$ is unbroken, $m_{\Phi_{1}}=m_{\Phi_{2}}$, so that $m_{\nu_{e}}=0 . S U(2)_{\nu}$ breaking effects, due to additional Higgs representations not shown here, give $m_{\Phi_{1,2}}^{2}=m_{\Phi}^{2} \pm \frac{1}{2} \Delta m_{\Phi}^{2}$. Therefore,

$$
\begin{aligned}
m_{\nu_{\mathrm{e}}} & \simeq \frac{f f^{\prime}}{16 \pi^{2}} m_{\tau} \cdot \frac{\Delta m_{\Phi}^{2}}{m_{\Phi}^{2}} \\
\mu_{\nu_{\mathrm{e}}} & \simeq \frac{e f f^{\prime}}{8 \pi^{2}} m_{\tau} \cdot \frac{1}{m_{\Phi}^{2}} \ln \frac{m_{\Phi}^{2}}{m_{\tau}^{2}}=e \frac{2 m_{\nu_{e}}}{\Delta m_{\Phi}^{2}} \ln \frac{m_{\Phi}^{2}}{m_{\tau}^{2}} .
\end{aligned}
$$

Demanding $m_{\nu_{e}}<20 \mathrm{eV}, \mu_{\nu_{\mathrm{e}}}>10^{-11} \mu_{B}$, one gets $\Delta m_{\Phi}^{2}<60 \mathrm{GeV}^{2}$ for $m_{\Phi}^{2} \simeq$ $(100 \mathrm{GeV})^{2}$.

The problem of the model [82] is that it is not easy to keep the $S U(2)_{\nu}$ breaking scale low without some fine-tuning of parameters in the Higgs potential. The advantage of this approach is that, since grand unified theories involve some extension of the standard model gauge group, one can hope that the extended electroweak symmetry needed here can come from some grand unified model in the course of symmetry breaking. Indeed, it has been shown by Deshpande and Pal [83] that, with some modifications, the above model can be derived from a grand unified group.

\subsubsection{Example: Quaternionic group ${ }^{\dagger}$}

Let us now give an example of how a discrete group can serve to implement Voloshin's idea. Consider the quaternionic group $Q$. It is a discrete group of eight 
elements, which can be called $\pm 1, \pm i, \pm j, \pm k$. The complete multiplication table can be deduced from the following rules: $i^{2}=j^{2}=k^{2}=-1, i j=k, j k=i, k i=j$. Thus, the group has two generators which we can take as $i$ and $j$, and all othor eloments can bo obtained as their products. There are five irreducthle representations of the group. Four of them are one dimensional, which we denote by $R_{1}$ through $R_{1}$. The other representation is two dimensional, which we call $D$. The representations of the generators $i$ and $j$ are given here:

\begin{tabular}{c|ccccc} 
& $R_{1}$ & $R_{2}$ & $R_{3}$ & $R_{4}$ & $D$ \\
\hline$i$ & 1 & 1 & -1 & -1 & $-i \sigma_{1}$ \\
$j$ & 1 & -1 & 1 & -1 & $-i \sigma_{2}$
\end{tabular}

The $D$ representation has the important property that $D \times D=\left(R_{1}\right)_{\text {antigymm }}+\left(R_{2}+\right.$ $\left.R_{3}+R_{4}\right)_{\text {aymm. }}$. Thus, if fermions transform as $D$, magnetic moment will transform like $R_{1}$ which is the trivial representation, invariant under transformations of the quaternionic group. The mass term, on the other hand, will transform either as $R_{2}, R_{3}$ or $R_{4}$ - all of which are nontrivial. Thus, the group $Q$ can act in place of Voloshin's $S U(2)_{\nu}$ to suppress $m_{\nu}$ [79].

To see how that works, we assign the following representations of $S U(3)_{c} \times$ $S U(2)_{L} \times U(1)_{Y} \times Q$ for fermions:

$$
\begin{array}{cl}
\Psi_{L}=\left(\begin{array}{cc}
\nu_{e} & \nu_{\mu} \\
e & \mu
\end{array}\right)_{L} & :\left(1,2,-\frac{1}{2}, D\right) \\
e_{R} & \\
\mu_{R} & :\left(1,1,-1, R_{2}\right) \\
g_{L}, g_{R} & :\left(1,1,-1, R_{3}\right) \\
& :\left(3,1,-\frac{1}{3}, R_{4}\right)
\end{array}
$$

Here, $g$ is an extra vectorlike quark. The usual quarks are not relevant for what follows, so we omitted them. The Higgs bosons of the model are:

$$
\begin{array}{ll}
\varphi:\left(1,2, \frac{1}{2}, R_{1}\right), & \Phi:\left(1,2, \frac{1}{2}, D\right) \\
H:\left(\overline{3}, 2,-\frac{1}{6}, D\right), & H^{\prime}:\left(\overline{3}, 2, \frac{5}{6}, D\right) .
\end{array}
$$

The gauge invariant Lagrangian has the following terms involving the fermions:

$$
\begin{aligned}
-\mathcal{L}_{y}= & f_{e}(\bar{\Psi} \Phi)_{R_{\mathbf{2}}} e_{R}+f_{\mu}(\bar{\Psi} \Phi)_{R_{3}} \mu_{R} \\
& +m_{g} \bar{g}_{L} g_{R}+h(\bar{\Psi} H)_{R_{4}} g_{R}+h^{\prime}\left(\Psi H^{\prime}\right)_{R_{4}} g_{L}+\text { h.c. }
\end{aligned}
$$

and the Higgs potential contains the terms

$$
V=\lambda_{\varphi}\left(H H^{\prime *}\right)_{R_{1}} \varphi^{2}+\sum_{i} \lambda_{\Phi}^{i}\left(H H^{\prime *}\right)_{R_{i}}(\Phi \Phi)_{R_{i}}+\ldots
$$




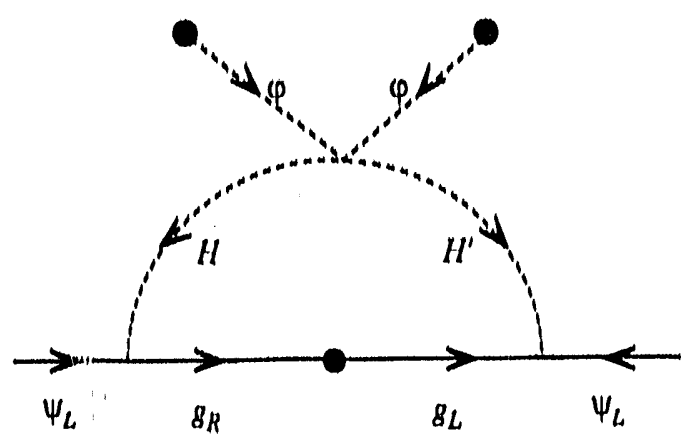

Figure 20: 1-loop diagrams involving Higgs boson exchange in the model of Ref. [79] which gives the dorninant contribution to neutrino magnetic moment when a photon line is attached to any internal line.

There are, of course, many other terms but they are not necessary in the ensuing discussion.

The magnetic moment arises from the diagram in Fig. 20. A rough estimate of the contribution is

$$
\mu_{\nu} \simeq \frac{e \lambda_{\varphi} h h^{\prime} m_{g}\langle\varphi\rangle^{2}}{16 \pi^{2} m_{I}^{4}}
$$

This diagram without the photon line, cannot contribute to $m_{\nu}$ since $\varphi$ is in the antisymmetric $R_{1}$ representation. But consider a similar diagram with $\Phi$ instead of $\varphi$ in the external legs. This gives a mass of order

$$
m_{1} \simeq \frac{\lambda_{\Phi} h h^{\prime} m_{g}\langle\Phi\rangle^{2}}{16 \pi^{2} m_{H}^{2}}
$$

Thus

$$
\frac{\mu_{\nu}}{m_{\nu}} \simeq \frac{e \lambda_{\varphi}}{\lambda_{\Phi}} \frac{\langle\varphi\rangle^{2}}{\langle\Phi\rangle^{2}} \cdot \frac{1}{m_{H}^{2}}
$$

If $\langle\Phi\rangle \ll\langle\varphi\rangle$, we can get a large magnetic moment with a small mass.

\section{Other ways of getting a large magnetic mo- ment}

So far, we discussed Voloshin's idea [72] of putting soxne extra symmetry which forbids neutino mass but does not forbid neutrino magnetic moment. There are some other ideas of how mass can be suppressed compared to the magnetic moment, which we discuss now. 


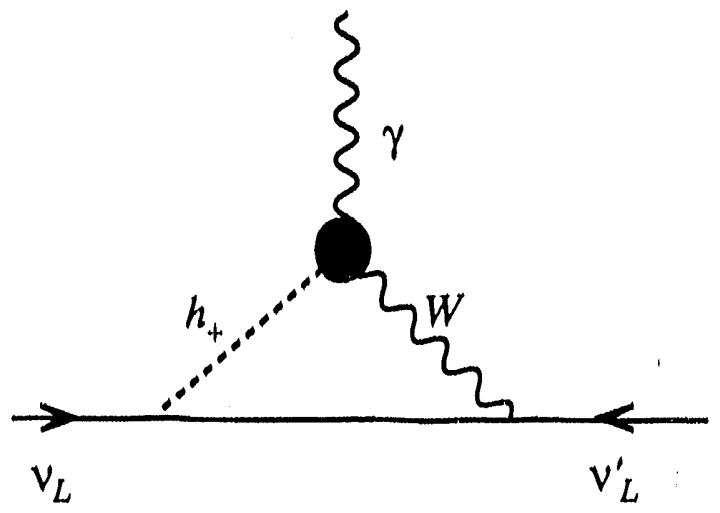

Figure 21: The spin suppression mechanism works if the dominant contribution to neutrino magnetic moment comes from a diagram like this. The blob represents an effective coupling generated by loops.

\subsection{Spin suppression}

Barr, Freire and Zee [84] have suggested another interesting scenario in which neutrino mass can be suppressed without suppressing magnetic moment. Suppose, for some reason, that the dominant contribution to magnetic moment comes through the generic diagram of Fig. 21, where the photon coupling is an effective vertex developed through quantum corrections. The helicity flip required to obtain the magnetic moment operator is obtained through the coupling of the scalar particle. The corresponding mass contribution is obtained by stripping Fig. 21 of the photon line. However, one this is done, we see that the blob involves a spin-0 particle turning into a spin-1 particle. This would be impossible if we have the transverse components of the vector particle connecting to the blob. However, the longitudinal component of the vector particle is really a spin-0 particle, so it can connect to the blob. In short, the vector line in the mass diagram must involve only the longitudinal component, i.e, the unphysical Higgs. However, the couplings of unphysical Higgs bosons can be derived from the gauge couplings irrespective of the details of the Higgs content of the model. For couplings with fermions, one obtains that the coupling involves factors $m_{f} / M_{W}$, where $m_{f}$ is a generic fermion mass. Since all known leptons are much lighter than $M_{W}$, this gives a suppression for the mass diagram. In the magnetic moment diagram, however, the transverse components of the vector field appear in general, and so the coupling is not suppressed. Moreover, since in the mass diagram both the vertices on the fermion line flip helicity, one needs another factor of lepton mass to obtain a net flip of helicity. Thus, the suppression of the mass diagram is really $m_{\ell}^{2} / M_{W}^{2}$. Thus, the bound of Eq. (10.4) is modified to $m_{\ell} \lesssim 1 \mathrm{GeV}$, which is surely satisfied for the electron and the muon, 


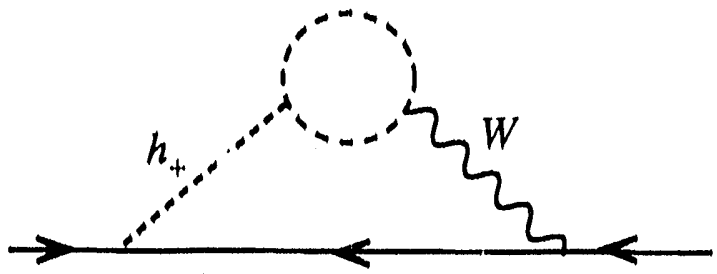

$v_{l ' L} \quad l_{L}$

$v_{L L}$

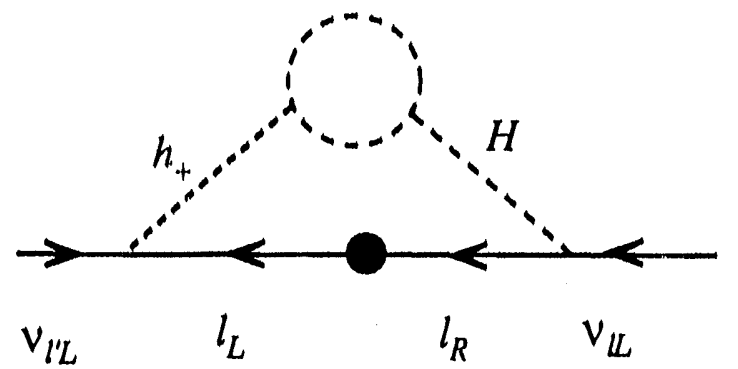

$V_{1 L}$

Figure 22: Adding a photon line to some internal line of thrsc diagrams give the dominant contribution to neutrino magnetic moment in the model of Ref. [84]. The scalar loops consist of Higgs doublets, and $H$ is the unphysical Higgs eaten up by the $W$.

and presumably also by the tauon.

To implement this idea, one therefore needs a model where a diagram like Fig. 21 indeed gives the dominant contribution to the magnetic moment. Barr, Freire and Zee [84] observed that they do not have to invent a new model for this. A model of neutrino mass, proposed long time ago by Zee [85], already has this property.

The model is based on the standard model gauge group $S U(2)_{L} \times U(1)_{Y}$, and contains several Higgs doublets (three are necessary to obtain large neutrino magnetic noment) and a charged field $h_{+}$which is a singlet of $S U(2)_{L}$. There are no right handed neutrinos. In Fig. 22, we show diagrams which give the dominant contributions to neutrino magnetic moment because they are unsuppressed by powers of $m_{\ell} / M_{W}$ :

$$
\mu_{\ell \ell^{\prime}} \sim \frac{e M_{0}}{\left(16 \pi^{2}\right)^{2}} \frac{f_{\ell \ell^{\prime}}}{M^{2}}
$$

where $f_{\ell \ell^{\prime}}$ is the Yukawa coupling matrix of $h_{+}$as defined in Eq. $(9.4), M_{0}$ is the trilinear coupling of $h_{+}$with two Higgs doublets, and $M$ is some heavy mass characteristic of the loop. For $M \sim M_{0} \sim 100 \mathrm{GeV}, f \sim 10^{-1}$, we can obtain magnetic moments of order $10^{-11} \mu_{B}$.

The mass contribution from these diagrams is the matrix

$$
m_{\ell \ell^{\prime}} \sim \frac{M_{0}}{\left(16 \pi^{2}\right)^{2}} \frac{f_{\ell \ell^{\prime}}\left(m_{\ell}^{2}-m_{\ell^{\prime}}^{2}\right)}{M^{\prime 2}},
$$

where $m_{\ell}$ and $m_{\ell^{\prime}}$ are the charged lepton masses. The quantity $M^{\prime}$ appearing in this expression involves mass differences of the charged and neutral components of doublet Higgs fields. If this splitting is small, it provides a further suppression in neutrino mass [84]. 


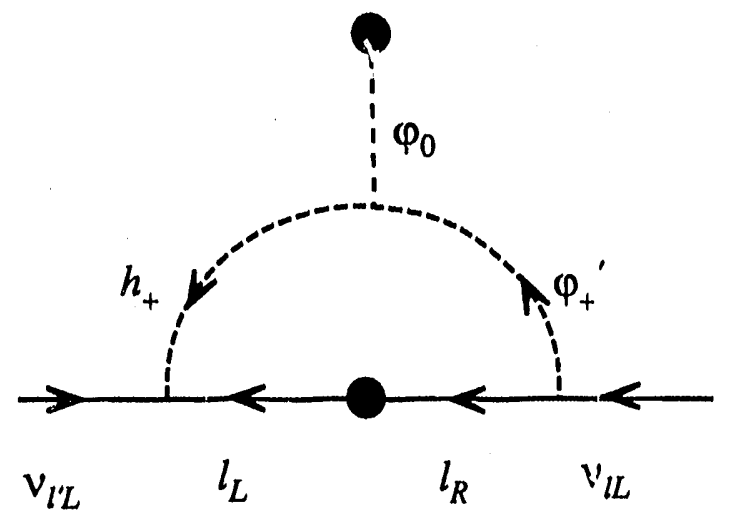

Figure 23: 1-loop contributions to neutrino mass Zee's model.

It should be commented that neutrino masses in this model can come from the 1-loop diagram of Fig. 23. These contributions are not as suppressed as the 2loop diagrams since the loop integration factors are not as small. But the doublets involved in this diagram have to be different because of $S U(2)_{L}$ symmetry. So, if we choose a basis in which only one Higgs doublet has a nonzero vev, and if the fermions couple only to this doublet, Fig. 23 vanishes. This can be achieved by imposing some discrete symmetries on the model [84].

\subsection{Vacuum structure}

Choudhury and Sarkar [86] have discussed a model where mass is suppressed for a different reasors. The essential features of the model can be summarized as follows.

Suppose we have a symmetry in our model which forbids both mass and magnetic moment. Both mass and magnetic moment can therefore be generated only in the symmetry breaking process. However, as we discussed in Sec. 10.3, the magnetic moment operator and the mass operator transform like different representations of the flavor group. Thus, they cannot be generated by a single vev. Mass arises from a vev transforming like the symmetric representation, and magnetic moment from vev like the antisymmetric representation. But suppose the vacuum structure of the model is such that the antisymmetric scalars have nonzero vev but the symmetric scalars are either absent altogether or do not acquire vev. In this case, one obtains magnetic moment but no mass.

As an illustrative example, Choudhury and Sarkar presented [86] a model based

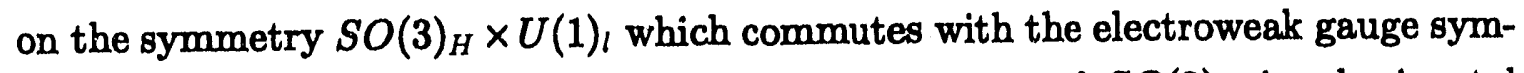
metry. The $U(1)_{l}$, here is the lepton number symmetry and $S O(3)_{H}$ is a horizontal symmetry. Whether they are gauged or not is of no importance here. Leptonic 
doublets transform as a triplet of $S O(3)_{H}$ :

$$
\left(\begin{array}{ccc}
\nu_{e} & \nu_{\mu} & \nu_{\tau} \\
e & \mu & \tau
\end{array}\right)_{L}
$$

which has lepton number 1 . There are no right handed neutrinos. Thus, magnetic moment terms must be transitional and transform like $(3,2)$ representation of $S O(3)_{H} \times U(1)_{l}$, whereas mass terms transform like $(5,2)$ or $(1,2)$. In the Higgs sector, there are particles that transform like $(3,2)$, which gives rise to magnetic moment. But no Higgs transforms like $(5,2)$ or $(1,2)$, so no mass can be generated.

\section{Chlorine vs Kamioka}

In the prelude to this part of the article, we mentioned that the time variation in the detected solar neutrino flux is apparent only in the chlorine experiment and not in Kamiokande data. We can ask ourselves whether it is possible to understand this discrepancy in a theoretical model.

The clue to the explanation might lie in the fact that the detection mechanisms for the two experiments are very different. The chlorine experiment detects $\nu_{e}$ by interaction with nucleons whereas the Kamiokande experiment detects neutrinos by their interaction with electrons. Recall that the key point is that a magnetic field rotates $\nu_{L}$ into $\nu_{R}$, and $\nu_{R}$ cannot be detected since we believe it has no interactions with normal matter.

But suppose [87] in a model $\nu_{R}$ interacts with $e^{-}$with strength comparable to $G_{F}$. Then the Kamiokande detector will detect both $\nu_{L}$ and $\nu_{R}$ with similar efficiency and will not find any net change. But if $\nu_{R}$ does not have interaction with quarks, ${ }^{37} \mathrm{Cl}$ detector will not see them.

To see how this can be realized in a model, add a Higgs doublet $\varphi^{\prime}$ to the standard model Higgs. There can be Yukawa interactions of the form $f \bar{\nu}_{R} \psi_{L} \varphi^{\prime}$, where $\psi_{L}$ is the lepton doublet. The coupling constant $f$ is not related to the neutrino mass if $\varphi^{\prime}$ has zero vev. The charged component of $\varphi^{\prime}$ will mediate $e-\nu_{e}$ interaction of strength $\frac{|f|^{2}}{4 M_{\varphi^{\prime}}^{2}}$. If this strength is comparable to the weak interaction strength, $G_{F}$, then the above situation is realized and we can understand why Kamiokande does not see time variation in the flux. 


\section{Part IV}

\section{Concluding remarks}

\section{Outlook for future experiments}

In Part I, we described that there is a discrepancy between solar neutrino flux esimated by solar model calculations and that detected by terrestrial experiments. In Parts II and III, we showed that this discrepancy can be solved by some properties of neutrinos like mass, mixing or magnetic moment. Such properties are yet unconfirmed from laboratory experirnents, but the range of magnitudes of these quantities required to solve the solar neutrino puzzle is largely unexplored.

Needless to say, one needs more data to explore the problem fully and confirm which solution, if any, is acceptable by all experiments. To this end, the Davis group and the Kamiokande collaboration continue taking more data. In addition, more detectors are being planned or being built. The radiochemical ${ }^{71} \mathrm{Ga}$ detector has recently been brought into operation by the SAGE group [9], although their results are tentative so far. The results of this detector will be crucial in our understanding of the solar neutrino puzzle since none of the earlier detectors could detect the low energy pp neutrinos, which constitute the bulk of the flux of neutrinos from the sun. Some new type of detectors are also in a planning stage. A heavy water detector at the Sudbury Neutrino Observatory (SNO) will detect neutrinos by their charged- as well as neutral-current interactions. The proposed Borex detector made of ${ }^{11} \mathrm{~B}$ would also be able to detect neutrinos via both charged- and neutral-current reactions with nuclei, as well as by electron scattering. If oscillation of $\nu_{e}$ to $\nu_{\mu}$ or $\nu_{\tau}$ is responsible for the solar neutrino problem, purely neutral current detectors should not see any depletion from the solar model calculations in the number of total neutrinos detected.

With new and plentiful data, one hopes to see the time variation and the energy dependence of the solar neutrino flux. The energy dependence, as argued before, can decide between different regions of the solution space obtained by resonant matter uscillation mechanium.

The time variation should tell us, first of all, whether the 11-year cycle of sunspot activities is anticorrelated with the solar neutrino flux. If the answer here is affirmative, that immediately selects out the neutrino magnetic moment as the solution of the puzzle.

Further refinement can see if there are time variations at smaller time scales. 
For example, if neutrinos have a magnetic moment, one expects a semiannual cycle in the solar neutrino flux. This is because the sun's magnetic equator is tilted at an angle of about $7^{\circ}$ with the earth's orbit. Twice a year - in June and December - the earth comes on the plane of the solar equator where the magnetic field is zero, as can be implied by the absence of sunspots there. When this happens, the neutrinos coming out to the earth encounters hardly any magnetic field and therefore would not undergo precession. This will result in a high value of the number of neutrinos detected. In March and September, the number of neutrinos would be minimum.

If, on the other hand, resonant matter oscillation is responsible for the solar neutrino puzzle, one expects a diurnal variation in the solar neutrino flux. This is because at night, the neutrino"comes through the earth, and matter effects in the earth's interior would affect the neutrino flux.

So far, the data is not enough to establish any of these variations [88]. Knowledge of such time variations will help determine the mechanism responsible for the solar neutrino problem. If the solution lies in resonant matter oscillation and only two generation of neutrinos are involved, one can easily find the mass and mixing parameters consistent with the experiments in the manner described in the text. If three generations are involved, one needs a better understanding of the non-adiabatic effects or needs to tackle them numerically.

If magnetic moment is responsible for the solar neutrino problem, then unfortunately it would be difficult to make precise estimates of the survival probability in any experiment since the magnetic fields in the sun are not very well known, as we mentioned earlier. This will call for better understanding of magnetohydrodynamic phenomena in the sun.

\section{Outlook for Physics beyond the standard model}

The solar neutrino puzzle can be summarized as follows: if the standard solar model calculations are reliable, the neutrino properties of the standard electroweak model cannot explain the results of the experiments to detect the neutrino flux. Assuming the reliability of the standard solar model as we have done throughout in this article, the solar neutrino puzzle than leads one to Particle Physics beyond the standard electroweak model. Indeed, the mass difference and mixing needed for neutrino oscillation, or the magnetic moment needed for spin precession - are all absent in the standard electroweak model.

It is however quite easy to accommodate neutrino mass and mixing by extending 
the standard model. For example, one can add a right handed neutrino to each generation of fermions. The neutrinos can then obtain mass through the Higgs mechanism, just like other fermions do. One can also extend the Higgs sector in many ways to obtain neutrino mass and mixing ${ }^{\sharp 7}$ All these models give rise to neutrino mixing in a natural way.

The mass squared difference required to solve the solar neutrino puzzle are very small. For the solution via vacuum oscillations, we need $\Delta \sim 10^{-10} \mathrm{eV}^{2}$. Matter enhanced oscillation can produce solutions for $\Delta$ as large as $\sim 10^{-4} \mathrm{eV}^{2}$. This, by itself, does not tell us about the masses as such. But, unless there is some minute cancellation between two masses, one would expect one eigenvalue close to $\Delta^{1 / 2}$ and another quite smaller. For $\Delta \lesssim 10^{-4} \mathrm{eV}^{2}$, one then expects $m_{\nu} \lesssim 10^{-2} \mathrm{eV}$, which is very small compared to the masses of any other fermion. The mixing angle needed to solve the solar neutrino puzzle can alzo be very small since matter effects can enhance it and produce large depletion. In a word, the matter enchanced oscillation solution really demands small modifications on the standard model.

The magnetic moment solution, on the other hand, requires some elaborate alterations of the standard model since, as we showed in Sec. 9.1, simple changes in the standard model produces magnetic moments much smaller than what is necessary. This is why most of our discussion in Part III was concerning the model building aspect to accommodate a large magnetic moment.

There is, however, another angle to this argument. Since the oscillation solutions require very small parameters, they will be almost impossible to find in any laboratory experiment. For example, if the neutrino mass is obtained through the see-saw mechanism and if the heavier of the neutrinos involved in oscillation is the $\nu_{\mu}$, the solutions for $\Delta \lesssim 10^{-4} \mathrm{eV}^{2}$ gives $M \gtrsim 10^{9} \mathrm{GeV}$ for the heavy mass occurring in the see-saw. This means that Physics beyond the standard model is really far beyond. If the heavier neutrino is $\nu_{\tau}$, the scale of new physics is even higher [90].

On the other hand, the magnetic moment solution always involves some new and unknown particles around the mass scale of $100 \mathrm{GeV}$ to $1 \mathrm{TeV}$. These would be accessible to the next generation of particle accelerators.

\section{Acknowledgements and apologies}

The article grew up from different lectures that I gave on the solar neutrino puzzle. I thank Partha Ghosh for inviting me to the 2nd Workshop on High Energy Physics Phenemenology held in Calcutta in January 1991 and asking me to give a talk. I thank Probir Roy and K. V. L. Sarma for an invitation at the Tata Institute of

\#7 For a descrintion of various models giving rise to neutrino mass, see, e.g., Ref. [89]. 
Fundamental Research for giving three lectures. I was inspired by the reaction of the mixed audience at our Institute of Theoretical Science where I gave three lectures at the request of Nilendra Deshpande. I thank him for organizing these talks as well as for numerous discussions. I also thank P. Roy, K. V. L. Sarma, S. M. Chitre, S. Rindani and A. Joshipura for discussions. I am indebted to Lincoln Wolfenstein for a careful reading of an early version of the article and making invaluable suggestions. I thank Fredrick Olness for many suggestions about efficient use of computers in preparing the manuscript. Since the literature on the subject is enormous, I have surely left out many papers in the references, for which I apologize. The research was supported by the Department of Energy grant DE-FG06-85ER40224.

\section{Appendices}

\section{A Laboratory bounds on neutrino oscillation pa- rameters}

The laboratory bounds on neutrino oscillations come from the following type of experiments. One takes a beam of known flavor from a reactor or an accelerator and lets it travel for some distance $x$. At that point, one checks whether the survival probability of the original flavor, or the conversion probability to another flavor, can be consistent with nonzero values of mixing and mass difference. So far, all confirmed results are negative. They can be summarized as follows: if $\Delta \lesssim 0.1 \mathrm{eV}^{2}$, there is no bound on the mixing angle, and if the mixing angle is smaller than about 0.05 , then there is no bound on $\Delta$. For details and references, see, e.g., Ref. [89]. Note that the solutions obtained for the solar neutrino problem pertains to much smaller values of $\Delta$ than $0.1 \mathrm{eV}^{2}$, so that all values of the mixing angles are allowed in this range by terrestrial experiments.

\section{B More rigorous derivation of dispersion rela- tions in medium}

In quantum field theory, the dispersion relation of a particle is given by the pole of its propagator. Thus, if we can find the full propagator of a neutrino within a medium, that can give us $\widetilde{H}$ of Eq. (4.15).

To do this, one calculates the self-energy of neutrinos in the thermal bath of the 

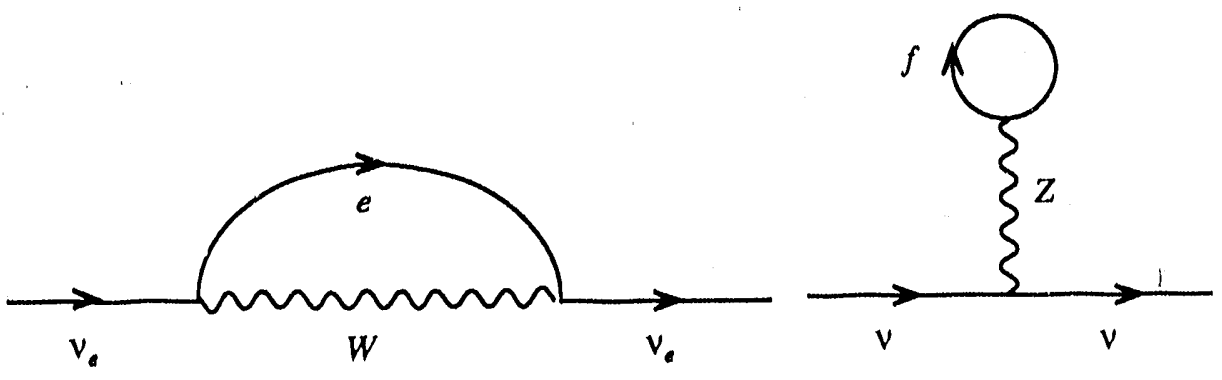

Figure 24: 1-loop self energy corrections to the neutrino propagator.

solar medium $[91,92,93]$. The full propagator is given in the form

$$
\frac{1}{\not p-m-\Sigma(p)} \text {. }
$$

In the lowest order, the contribution to $\Sigma(p)$ comes from the diagrams shown in Fig. 24. Considering just the charged current diagram for the moment, we obtain

$$
i \Sigma(p)=i\left(\frac{g}{\sqrt{2}}\right)^{2} \int \frac{d^{4} k}{(2 \pi)^{4}} \gamma_{\lambda} P_{L} i S_{e}(k) \gamma_{\rho} P_{L} \cdot \frac{i g^{\lambda \rho}}{M_{W}^{2}}
$$

where $S_{e}(k)$ is the propagator of the electron, and we have neglected the momentum dependence of the $W$-propagator. For the electron propagator, we use the effective propagator in the medium, which is

$$
i S_{e}(k)=\left(k+m_{e}\right)\left[\frac{i}{k^{2}-m_{e}^{2}}-2 \pi \delta\left(k^{2}-m_{e}^{2}\right) f_{F}(k \cdot u)\right],
$$

where

$$
f_{F}(x)=\frac{\Theta(x)}{e^{\beta(x-\mu)}+1}+\frac{\Theta(-x)}{e^{-\beta(x-\mu)}+1},
$$

where $\Theta$ is the step function which equals +1 if the argument is positive and is zero otherwise, $\mu$ is the chemical potential and $\beta^{-1}$ is the temperature of the background electrons.

The first term in the electron propagator in Eq. (B.3) gives infinite renormalization. This is present even in the vacuum and is eliminated by a suitable renormalization prescription. The second term gives

$$
\sqrt{2} G_{F}\left(n_{e^{-}}-n_{e^{+}}\right) \gamma_{0}
$$

which then easily gives Eq. (4.4). Similarly, one can evaluate the neutral current diagram. Note that for this derivation, we need not assume that velocity or spin has to vanish; as we did in the text. 


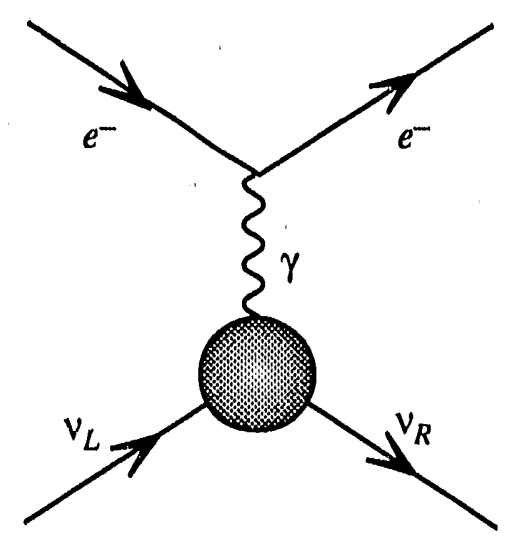

Figure 25: Electron-neutrino scattering through neutrino magnetic moment. The blob is the effective coupling of neutrinos with photons.

\section{Laboratory bounds on neutrino magnetic mo- ments}

Here, we give a rough estimate of the bounds on neutrino magnetic moment that can be obtained from laboratory experiments. The bounds arise because the neutrinos can scatter against electrons via photon exchango through the diagram of Fig. 25. The cross section calculated from this diagram can be easily estimated:

$$
\sigma_{\text {mag }} \sim e^{2} \mu^{2} \sim \frac{e^{4}}{m_{e}^{2}}\left(\frac{\mu_{i}}{\mu_{B}}\right)^{2},
$$

neglecting factors of order unity. The tree level $W$ and $Z$ exchange, on the other hand, gives

$$
\sigma_{W, Z} \sim \frac{g^{4}}{M_{W}^{4}} E m_{e}
$$

where $g$ is the SU(2) gauge coupling constant of the standard model, and $E$ is the neutrino energy, assumed much smaller than $M_{W}$. Since the standard model calculation fits scattering data very well, we can demand

$$
\sigma_{\mathrm{mag}} / \sigma_{W, Z} \leq 1 \text {. }
$$

This gives, since $g \sim e$,

$$
\left(\frac{\mu}{\mu_{B}}\right)^{2} \lesssim \frac{m_{e}^{3} E}{M_{W}^{4}} \sim 10^{-19}\left(\frac{E}{10 \mathrm{MeV}}\right)^{2}
$$

Reactor experiments have been performed upto neutrino energies of order of a few tens of MeV's. So, from Eq. (C.4), one gets

$$
\mu \leq 3 \times 10^{-10} \mu_{B} \text {. }
$$


If one does more careful analysis of individual experiments and the error bars in their measurements, one gets upper bounds ranging from $(4$ to 20$) \times 10^{-10} \mu_{B}$. There are also other bounds coming from astrophysical and cosmolngical considerations. For a summary of these bounds and references, see Ref. [89].

\section{References}

[1] D D Clayton: Principles of Stellar Evolution and Nucleosynthesis (McGrawHill 1968).

[2] S Turck-Chièze, S Cahen, M Cassé, C Doom: Astrophys. J. 335 (1988) 415.

[3] J N Bahcall, R K Ulrich: Rev. Mod. Phys. 60 (1988) 297.

[4] J N Bahcall, H Bethe: Phys. Rev. Lett. 65 (1990) 2233.

[5] J N Bahcall: Neutrino Astrophysics (Cambridge Univ. Press 1989).

[6] V Barger, R J N Philips, K Whisnant: Phys. Rev. D43 (1991) 1110.

[7] R Davis: in Neutrino '88, proceedings of the XIIIth International Conference on Neutrino Physics and Astrophysics, ed. J Schneps et al (World Scientific 1989) p. 518.

[8] Kamiokande Collaboration: Phys. Rev. Lett. 65 (1990) 1297.

[9] V N Gavrin, SAGE Experiment: talk at the Neutrino '90 conference, (Geneva 1990).

[10] T Kirsten, GALLEX Experiment: talk at the Neutrino '90 conference, (Geneva 1990).

[11] B Pontecorvo: JETP 6 (1958) 429.

[12] B Pontecorvo: Sov. Phys. JETP 6 (1966) 1305.

[13] V Barger, R J N Phillips, K Whisnant: Phys. Rev. Lett. 65 (1990) 3084.

[14] A Acker, S Pakvasa, J Pantaleone: Phys. Rev. D43 (1991) 1754.

[15] L Wolfenstein: Phys. Rev. D17 (1978) 2369.

[16] S P Mikheyev, A Yu Smirnov: Nuovo Cimento 9C (1986) 17. 
[17] T K Kuo, J Pantaleone: Rov. Mod. Phys. 61 (1989) 937.

[18] V Barger, R J N Phillips, K Whisnant: Phys. Rev. D34 (1986) 980.

[19] H Bethe: Phys. Rev. Lett. 56 (1986) 1305.

[20] A Messiah: in '86 Massive Neutrinos in Astrophysics and Particle Physics, ed. O Fackler \& J Tran Thanh Van (Éditions Frontières, Gif-sur-Yvette 1986), p. 373.

[21] S J Parke: Phys. Rev. Lett. 57 (1986) 1275.

[22] W C Haxton: Phys. Rev. D35 (1987) 2352.

[23] V Barger, N G Deshpande, R J N Phillips, P B Pal, K Whisnant: Phys. Rev. D43 (1991) 1759.

[24] L Landau: Phys. Z. Sowjetunion 2 (1932) 46.

[25] C Zener: Proc. Roy. Soc. 137 (1932) 696.

[26] E C G Stückelberg: Helv. Phys. Acta 5 (1932) 369.

[27] W C Haxton: Phys. Rev. Lett. 57 (1986) 1271.

[28] L D Landau, L M Lifshitz: Quantum Mechanics, 3rd edition (Pergamon Press, Oxford 1977).

[29] P Pizzochero: Phys. Rev. D36 (1987) 2293.

[30] T K Kuo, J Pantaleone: Phys. Rev. D39 (1989) 1930.

[31] J M Gelb, S P Rosen: Phys. Rev. D34 (1986) 969.

[32] T Kaneko: Prog. Theor. Phys. 78 (1987) 532.

[33] S Toshev: Phys. Lett. B196 (1987) 170.

[34] S T Petcov: Phys. Lett. B200 (1988) 373.

[35] D Notzold: Phys. Rev. D36 (1987) 1625.

[36] M Ito, T Kaneko, M Nakagawa: Prog. Theor. Phys. 79 (1988) 13.

[37] J N Bahcall, W C Haxton: Phys. Rev. D40 (1989) 931.

[38] T K Kuo, J Pantaleone: Phys. Rev. D41 (1990) 3842. 
[39] T K Kuo, J Pantaleone: Mod. Phys. Lett. A6 (1991) 15.

[40] E W Kolb, M S Turner, T P Walker: Phys. Lett. B175 (1986) 478.

[41] S J Parke, T P Walker: Phys. Rov, Lett. 57 (1986) 2322.

[42] H A Bethe: Phys, Rev, Lett. 63 (1989) 837.

[43] Kamiokande Collaboration: Phys. Rev. Lett. 65 (1990) 1301.

[44] S M Bilenky, B Pontecorvo: Phys. Rep، 41 (1978) 225.

[45] V Barger, K Whisnant, S Pakvasa, R J N Phillips: Phys. Rov, D22 (1980) 2718.

[46] H W Zaglauer, K H Schwarzer: Phys. Lett. 198B (1987) 556.

[47] T K Kuo, J Pantaleone: Phys. Rev, Lett. 57 (1986) 1805.

[48] T K Kuo, J Pantaleone: Phys. Rev, D35 (1987) 3432.

[49] H W Zaglauer, K H Schwarzer: Z. Phys. C40 (1988) 273.

[50] A Joshipura, M V N Murthy: Phys. Rev. D37 (1988) 1374.

[51] A Baldini, G F Giudice: Phys. Lett. 186B (1987) 211.

[52] C W Kim, W K Sze: Phys. Rev. D35 (1987) 1404.

[53] A Bottino, J Ingham, C W Kim: Phys. Rev. D39 (1989) 909.

[54] S T Petcov, S Toshev: Phys. Lett.' 187B (1987) 120.

[55] S T Petcov: Phys. Lett. 214B (1988) 259.

[56] S P Mikheyev, A Y Smirnov: Phys. Lett. 200B (1988) 560.

[57] C W Kim, S Nussinov, W K Sze: Phys. Lett. 184B (1987) 403.

[58] J N Bahcall, W H Press: AstroPhys. J. 370 (1991) 730.

[59] R Cisneros: Astrophys. Space Sci. 10 (1971) 87.

[60] M Voloshin, M Vysotskii, L B Okun: JETP 64 (1986) 446.

[61] M Voloshin, M Vysotskil, L B Okun: Sov. J. Nucl. Phys. 44 (1986) 440.

[62] E Akhmedov, M Y Khlopov: Mod. Phys. Lett. A3 (1988) 451. 
[63] O S Lim, W Marciano: Phys، Rev, D37 (1988) 1368.

[64] J Pulldo: Phys. Lett, B251 (1990) 305.

[65] H Minakata, H Nunokawa: Phys. Rov, D43 (1991) 297.

[66] W A Dzlembowskd, P R Goode: Astrophys. J. 347 (1989) 540.

[67] J Vidal, J Wudka: Phys. Lett. 249B (1990) 473.

[68] B W Lee, R E Shrock: Phys. Rev، D16 (1977) 1444.

[69] M Fukugita, T Yanagida: Phys. Rev. Lett. 58 (1987) 1807.

[70] K S Babu, V S Mathur: Phys. Lett. 196B (1987) 218.

[71] S Rajpoot: Phys. Lett. 237 (1990) 77.

[72] M B Voloshin: Sov. J. Nucl. Phys. 48 (1988) 512.

[73] M Leurer, N Marcus: Phys. Lett. B237 (1990) 81.

[74] K S Babu, R N Mohapatra: Phys. Rov. Lett. 63 (1989) 228.

[75] K S Babu, R N Mohapatra: Phys. Rov. D42 (1990) 3778.

[76] H Georgi, L Randall: Phys. Lett. 244B (1990) 196.

[77] K S Babu, R N Mohapatra: Phys. Rev. Lett. 64 (1990) 1705.

[78] G Ecker, W Grimus, H Neufeld: Phys. Lett. B232 (1990) 217.

[79] D Chang, W Y Keung, G Senjanović: Phys. Rev. D42 (1990) 1599.

[80] D Chang, W Y Keung, S Llpovaca, G Senjanović: Preprint CERN-TH-602791 (March 1991).

[81] R Barbierl, R N Mohapatra: Phys. Lett. 218B (1989) 225.

[82] J Liu, Phys. Lett. B225 (1989) 148.

[83] N G Deshpande, P B Pal: Preprint OITS-467 (May 1991).

[84] S M Barr, E M Freire, A Zee: Phys. Rev. Lett. 65 (1990) 2626.

[85] A Zee: Phys. Lett. 93.B (1980) 389.

[86] D Choudhury, U Sarkar: Phys. Lett. 235B (1990) 113. 
[87] M Fukugita, T Janagida: Phys, Rov. Lett. 65 (1990) 1975.

[88] Kamiolcande Collaboration: Phys. Rev. Lett. 66 (1991) 9.

[89] R N Mohapatra, P B Pal: Massive Neutrinos in Physics and Astrophysics (World Sclentific 1991).

[90] P Langacker, S T Petcov, G Steigman, S Toshev: Nucl. Phys. B282 (1987) 589.

[91] D Nötzold, G Raffelt: Nucl. Phys. B307 (1988) 924.

[92] P B Pal, T N Pham: Phys. Rev، D40 (1989) 259.

[93] J F Nleves: Phys. Rev. D40 (1989) 866. 

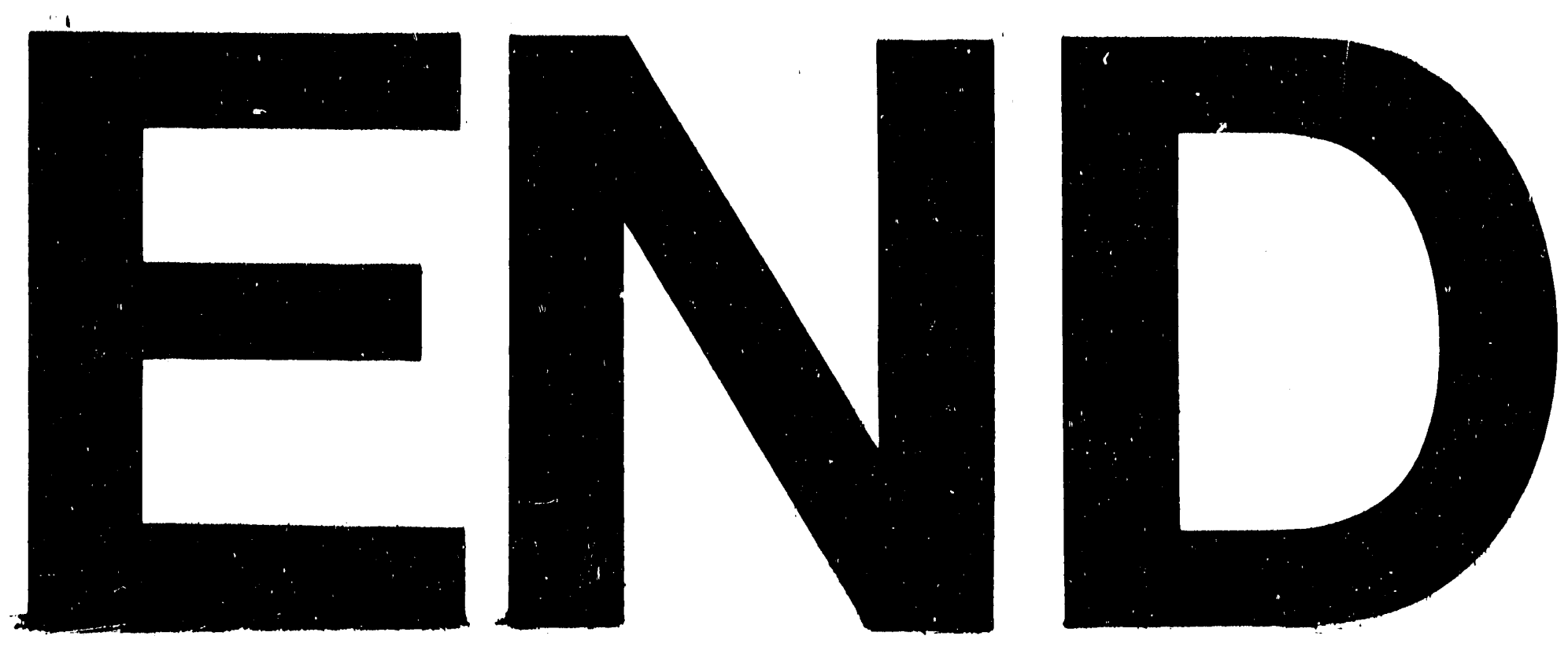

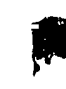

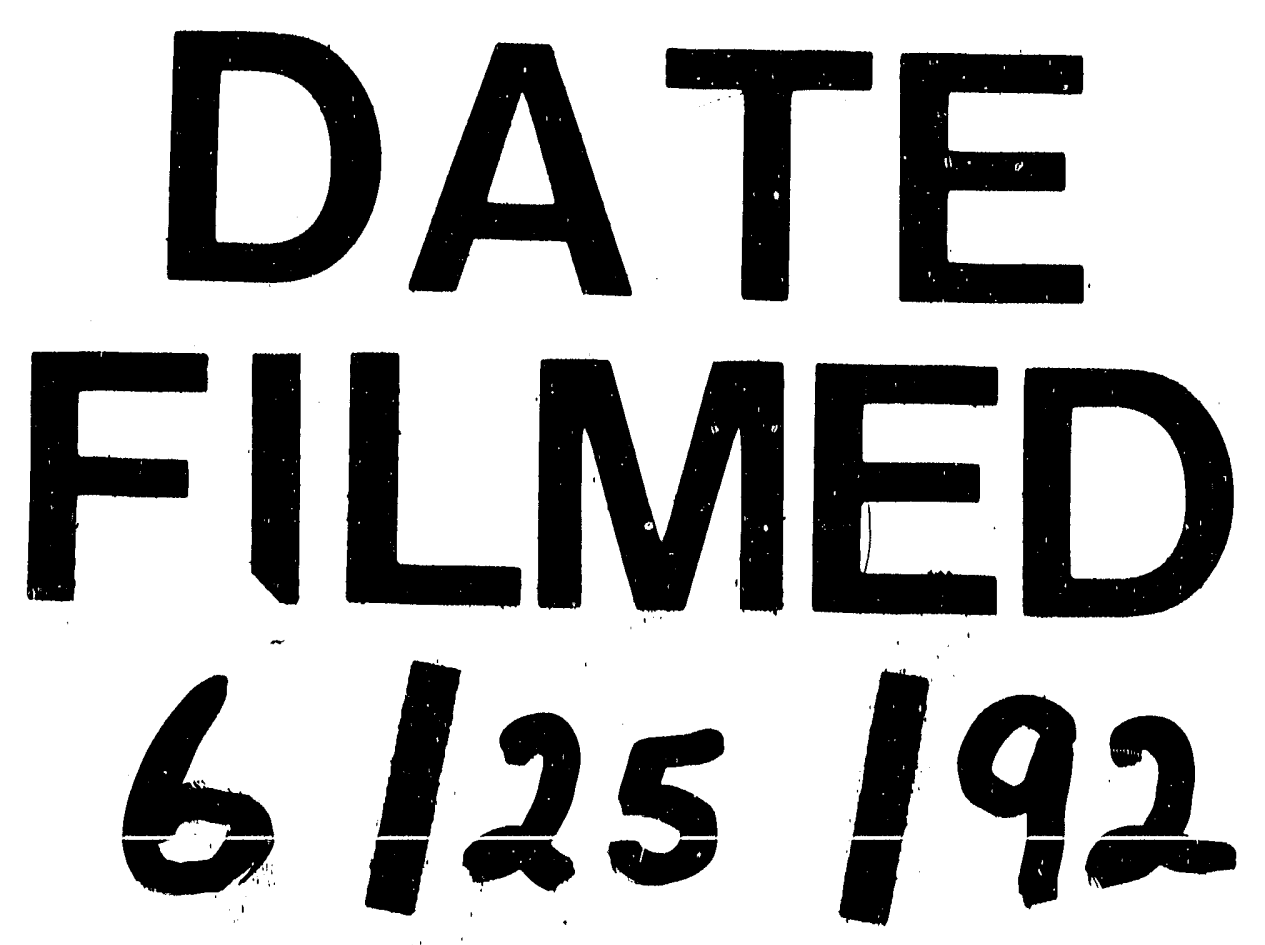

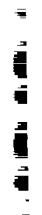

